\title{
THE SDSS-IV EXTENDED BARYON OSCILLATION SPECTROSCOPIC SURVEY: OVERVIEW AND EARLY DATA
}

Kyle S. Dawson ${ }^{1}$, Jean-Paul Kneib ${ }^{2,3}$, Will J. Percival ${ }^{4}$, Shadab Alam ${ }^{5}$, Franco D. Albareti ${ }^{6,70}$, Scott F. Anderson ${ }^{7}$, Eric Armengaud ${ }^{8}$, Éric Aubourg ${ }^{9}$, Stephen Bailey $^{10}$, Julian E. Bautista ${ }^{1}$, Andreas A. Berlind ${ }^{11}$,

Matthew A. Bershady ${ }^{12}$, Florian Beutler ${ }^{10}$, Dmitry Bizyaev ${ }^{13,14,15}$, Michael R. Blanton ${ }^{16}$, Michael Blomevist ${ }^{17}$, Adam S. Bolton ${ }^{1}$, Jo Bovy ${ }^{18}$, W. N. Brandt ${ }^{19,20,21}$, Jon Brinkmann ${ }^{13}$, Joel R. Brownstein ${ }^{1}$, Etienne Burtin ${ }^{8}$, N. G. Busca ${ }^{9}$, Zheng Cai ${ }^{22}$, Chia-Hsun Chuang ${ }^{6}$, Nicolas Clere ${ }^{23}$, Johan Comparat ${ }^{6,24,71}$, Frances Cope ${ }^{13}$, Rupert A. C. Croft ${ }^{5}$, Irene Cruz-Gonzalez ${ }^{25}$, Luiz N. da Costa ${ }^{26,27}$, Marie-Claude Cousinou ${ }^{28}$, Jeremy Darling ${ }^{29}$, axel de la Macorra ${ }^{25}$, Sylvain de la Torre ${ }^{3}$, Timothée Delubac ${ }^{2}$, Hélion du Mas des Bourboux ${ }^{8}$, Tom Dwelly ${ }^{23}$, Anne Ealet $^{28}$, Daniel J. Eisenstein ${ }^{30}$, Michael Eracleous ${ }^{19,20,21}$, S. Escoffier ${ }^{28}$, XiaOhui Fan ${ }^{22}$, Alexis Finoguenov ${ }^{31}$, Andreu Font-Ribera ${ }^{10}$, Peter Frinchaboy ${ }^{32}$, Patrick Gaulme ${ }^{13}$, Antonis Georgakakis ${ }^{23}$, Paul Green ${ }^{30}$, Hong Guo ${ }^{1,33}$, Julien GuY ${ }^{34}$, Shirley Ho ${ }^{5}$, Diana Holder ${ }^{13}$, Joe Huehnerhoff ${ }^{13}$, Timothy Hutchinson ${ }^{1}$, Yipeng Jing ${ }^{35}$, Eric Jullo ${ }^{3}$, Vikrant Kamble ${ }^{1}$, Karen Kinemuchi ${ }^{13,14}{ }^{\text {, David Kirkby }}{ }^{17}$, Francisco-Shu Kitaura ${ }^{36}$, Mark A. Klaene ${ }^{13}$,

Russ R. Laher ${ }^{37}$, Dustin Lang ${ }^{5}$, Pierre Laurent ${ }^{8}$, Jean-Marc Le GofF $^{8}$, Cheng Li $^{33}$, Yu Liang ${ }^{38}$, Marcos Lima ${ }^{27,39}$, Qiufan Lin ${ }^{38}$, Weipeng Lin ${ }^{33,40}$, Yen-Ting Lin ${ }^{41}$, Daniel C. Long ${ }^{13}$, Britt Lundgren ${ }^{12,72}$, Nicholas MacDonalD ${ }^{7}$, Marcio Antonio Geimba Maia ${ }^{26,27}$, Elena Malanushenko ${ }^{13,14}$, Viktor Malanushenko ${ }^{13,14}{ }^{14}$, Vivek Mariappan ${ }^{1}$,

Cameron K. McBride ${ }^{30}$, ian D. McGreer ${ }^{22}$, Brice Ménard ${ }^{42,43}$, Andrea Merloni ${ }^{23}$, Andres Meza ${ }^{44}$,

antonio D. Montero-Dorta ${ }^{1}$, Demitri Muna ${ }^{45}$, Adam D. Myers ${ }^{46}$, Kirpal Nandra $^{23}$, Tracy Naugle ${ }^{13}$,

Jeffrey A. Newman ${ }^{47}$, Pasquier Noterdaeme ${ }^{48}$, Peter Nugent ${ }^{10,49}$, Ricardo Ogando ${ }^{26,27}$, Matthew D. Olmstead ${ }^{50}$, Audrey Oravetz ${ }^{13,14}$, Daniel J. Oravetz ${ }^{13,14}$, Nikhil Padmanabhan ${ }^{51}$, Nathalie Palanque-Delabrouille ${ }^{8}$,

Kaike Pan ${ }^{13,14}$, John K. Parejko ${ }^{5}$, Isabelle Pâris ${ }^{52}$, John A. Peacock ${ }^{53}$, Patrick Petitjean ${ }^{48}$, Matthew M. Pieri ${ }^{3}$, Alice Pisani ${ }^{28,48,54}$, Francisco Prada ${ }^{6,55,56}$, Abhishek Prakash ${ }^{47}$, Anand Raichoor ${ }^{8}$, Beth Reid ${ }^{10}$, James Rich ${ }^{8}$, Jethro Ride ${ }^{23}$, Sergio Rodriguez-Torres ${ }^{6}$, Aurelio Carnero Rosell ${ }^{26,27}$, Ashley J. Ross ${ }^{4,57}$, Graziano Rossi ${ }^{58}$

John Ruan ${ }^{7}$, Mara Salvato ${ }^{23}$, Conor Sayres ${ }^{7}$, Donald P. Schneider ${ }^{19,20}$, David J. Schlegel ${ }^{10}$, Uros Seljak ${ }^{10,49,59,60}$, Hee-Jong Seo ${ }^{61}$, Branimir Sesar ${ }^{62}$, Sarah Shandera ${ }^{20}$, Yiping Shu ${ }^{1}$, Anže Slosar ${ }^{63}$, Flavia Sobreira ${ }^{27,64}$,

Alina Streblyanska ${ }^{65,66}$, Nao Suzuki ${ }^{43}$, Donna Taylor ${ }^{1}$, Charling TaO ${ }^{28,38}{ }^{\text {, Jeremy L. Tinker }}{ }^{16}$, Rita Tojeiro ${ }^{4}$,

Mariana Vargas-Magaña ${ }^{67}$, Yuting Wang ${ }^{4,68}$, Benjamin A. Weaver ${ }^{16}$, David H. Weinberg ${ }^{57,69}$, Martin White ${ }^{10,49,59}$,

W. M. Wood-Vasey ${ }^{47}$, Christophe Yeche ${ }^{8}$, ZhongXu Zhai ${ }^{16}$, Cheng Zhao ${ }^{38}$, Gong-Bo Zhao ${ }^{4,68}$, Zheng Zheng ${ }^{1}$, Guangtun Ben Zhu ${ }^{42,73}$, and Hu Zou ${ }^{68}$

${ }^{1}$ Department of Physics and Astronomy, University of Utah, Salt Lake City, UT 84112, USA; kdawson@astro.utah.edu

${ }^{2}$ Laboratoire dástrophysique, Ecole Polytechnique Fédérale de Lausanne Observatoire de Sauverny, 1290 Versoix, Switzerland

${ }^{3}$ Aix Marseille Université, CNRS, LAM (Laboratoire d'Astrophysique de Marseille), UMR 7326, F-13388, Marseille, France

${ }^{4}$ Institute of Cosmology \& Gravitation, Dennis Sciama Building, University of Portsmouth, Portsmouth, PO1 3FX, UK

${ }^{5}$ Bruce and Astrid McWilliams Center for Cosmology, Department of Physics, Carnegie Mellon University, 5000 Forbes Ave, Pittsburgh, PA 15213, USA

${ }^{6}$ Instituto de Física Teórica, (UAM/CSIC), Universidad Autónoma de Madrid, Cantoblanco, E-28049 Madrid, Spain

${ }^{7}$ Department of Astronomy, University of Washington, Box 351580, Seattle, WA 98195, USA

${ }^{8}$ CEA, Centre de Saclay, Irfu/SPP, F-91191 Gif-sur-Yvette, France

9 APC, University of Paris Diderot, CNRS/IN2P3, CEA/IRFU, Observatoire de Paris, Sorbonne Paris Cite, France

${ }^{10}$ Lawrence Berkeley National Laboratory, One Cyclotron Road, Berkeley, CA 94720, USA

${ }^{11}$ Department of Physics and Astronomy, Vanderbilt University, PMB 401807, 2401 Vanderbilt Place, Nashville, TN 37240, USA

${ }^{12}$ University of Wisconsin-Madison, Department of Astronomy, 475 N. Charter St., Madison WI 53703, USA

${ }^{13}$ Apache Point Observatory, P.O. Box 59, sunspot, NM 88349, USA

${ }^{14}$ Department of Astronomy, MSC 4500, New Mexico State University, P.O. Box 30001, Las Cruces, NM 88003, USA

${ }^{15}$ Sternberg Astronomical Institute, Moscow State University, Moscow, Russia

${ }^{16}$ Center for Cosmology and Particle Physics, Department of Physics, New York University, 4 Washington Place, New York, NY 10003, USA

${ }^{17}$ Department of Physics and Astronomy, University of California, Irvine, CA 92697, USA

${ }^{18}$ Department of Astronomy and Astrophysics, University of Toronto, 50 St. George Street, Toronto, ON, M5S 3H4, Canada

${ }^{19}$ Department of Astronomy and Astrophysics, 525 Davey Laboratory, The Pennsylvania State University, University Park, PA 16802, USA

${ }^{20}$ Institute for Gravitation and the Cosmos, The Pennsylvania State University, University Park, PA 16802, USA

${ }^{21}$ Department of Physics, The Pennsylvania State University, University Park, PA 16802, USA

${ }^{22}$ Steward Observatory, 933 North Cherry Avenue, Tucson, AZ 85721, USA

${ }^{23}$ Max-Planck-Institut für Extraterrestrische Physik, Giessenbachstraße, D-85748 Garching, Germany

${ }_{25}^{24}$ Departamento de Fisica Teorica, Universidad Autónoma de Madrid, Cantoblanco, E-28049 Madrid, Spain

${ }^{25}$ Instituto de Astronomía, Universidad Nacional Autonoma de Mexico, A.P. 70-264, 04510, D.F., Mexico

${ }^{26}$ Observatório Nacional, Rua Gal. José Cristino 77, Rio de Janeiro, RJ-20921-400, Brazil

${ }^{27}$ Laboratório Interinstitucional de e-Astronomia,_-LIneA, Rua Gal. José Cristino 77, Rio de Janeiro, RJ-20921-400, Brazil

${ }^{28}$ Aix-Marseille Université, CNRS/IN2P3, CPPM UMR 7346, F-13288 Marseille, France

${ }^{29}$ Center for Astrophysics and Space Astronomy, Department of Astrophysical and Planetary Sciences, University of Colorado, 389 UCB, Boulder, CO 80309, USA

${ }_{31}$ Harvard-Smithsonian Center for Astrophysics, Harvard University, 60 Garden St., Cambridge MA 02138, USA

${ }^{31}$ Department of Physics, University of Helsinki, Gustaf Hällströmin katu 2a, FI-00014 Helsinki, Finland

32 Dept. of Physics \& Astronomy, Texas Christian University, 2800 South University Dr., Fort Worth, TX 76129, USA

${ }_{33}$ Shanghai Astronomical Observatory, Chinese Academy of Science, 80 Nandan Road, Shanghai 200030, China

${ }^{34}$ LPNHE, CNRS/IN2P3, Université Pierre et Marie Curie Paris 6, Université Denis Diderot Paris 7, 4 place Jussieu, F-75252 Paris CEDEX, France 
${ }^{35}$ IFSA Collaborative Innovation Center, Department of Physics and Astronomy, Shanghai Jiao Tong University, Shanghai, 200240, China

${ }^{36}$ Leibniz-Institut für Astrophysik Potsdam (AIP), An der Sternwarte 16, D-14482 Potsdam, Germany

${ }^{37}$ Spitzer Science Center, California Institute of Technology, M/S 314-6, Pasadena, CA 91125, USA ${ }^{38}$ Tsinghua Center for Astrophysics, Tsinghua University, Beijing 100084, China

${ }^{39}$ Departamento de Física Matemática, Instituto de Física, Universidade de São Paulo, CP 66318, CEP 05314-970, São Paulo, SP, Brazil

${ }^{40}$ School of Astronomy and Space Science, Sun Yat-sen University, Guangzhou, 510275, China

${ }^{41}$ Institute of Astronomy and Astrophysics, Academia Sinica, Taipei 10617, Taiwan

${ }^{42}$ Center for Astrophysical Sciences, Department of Physics and Astronomy, Johns Hopkins University, 3400 North Charles Street, Baltimore, MD 21218, USA

${ }^{43}$ Kavli Institute for the Physics and Mathematics of the Universe, Todai Institutes for Advanced Study, The University of Tokyo, Kashiwa, 277-8583, Japan

${ }^{44}$ Departamento de Ciencias Fisicas, Universidad Andres Bello, Av. Republica 220, Santiago, Chile

${ }^{45}$ Department of Physics and Center for Cosmology and Astro-Particle Physics, Ohio State University, Columbus, OH 43210, USA

${ }^{46}$ Department of Physics and Astronomy, University of Wyoming, Laramie, WY 82071, USA

${ }^{47}$ Department of Physics and Astronomy and PITT PACC, University of Pittsburgh, Pittsburgh, PA 15260, USA

${ }^{48}$ UPMC-CNRS, UMR7095, Institut dAstrophysique de Paris, 98bis Boulevard Arago, F-75014, Paris, France

${ }^{49}$ Department of Astronomy, University of California, Berkeley, CA 94720, USA

${ }^{50}$ Department of Chemistry and Physics, Kings College, Wilkes Barre, PA, 18711, USA

${ }^{51}$ Yale Center for Astronomy and Astrophysics, Yale University, New Haven, CT, 06520, USA

${ }^{52}$ INAF-Osservatorio Astronomico di Trieste, Via G. B. Tiepolo 11, I-34131 Trieste, Italy

${ }^{53}$ Institute for Astronomy, University of Edinburgh, Royal Observatory, Edinburgh EH9 3HJ, UK

${ }^{54}$ Sorbonne Universités, UPMC (Paris 06), UMR7095, Institut d'Astrophysique de Paris, 98bis Bd. Arago, F-75014, Paris, France

${ }_{55}$ Campus of International Excellence UAM+CSIC, Cantoblanco, E-28049 Madrid, Spain

${ }^{56}$ Instituto de Astrofísica de Andalucía (CSIC), Glorieta de la Astronomía, E-18080 Granada, Spain

${ }^{57}$ Center for Cosmology and Astro-Particle Physics, Ohio State University, Columbus, OH 43210, USA

${ }^{58}$ Department of Astronomy and Space Science, Sejong University, Seoul, 143-747, Korea

${ }^{59}$ Department of Physics, University of California, Berkeley, CA 94720, USA

${ }^{60}$ Berkeley Center for Cosmological Physics, LBL and Department of Physics, University of California, Berkeley, CA 94720, USA

${ }^{61}$ Department of Physics and Astronomy, Ohio University, 251B Clippinger Labs, Athens, OH 45701

${ }^{62}$ Max Planck Institute for Astronomy, Königstuhl 17, D-69117 Heidelberg, Germany

${ }^{63}$ Bldg 510 Brookhaven National Laboratory Upton, NY 11973, USA

${ }^{64}$ Fermi National Accelerator Laboratory, P.O. Box 500, Batavia, IL 60510, USA

${ }^{65}$ Instituto de Astrofísica de Canarias (IAC), C/Vía Láctea, s/n, E-38200, La Laguna, Tenerife, Spain

${ }^{66}$ Dpto. Astrofísica, Universidad de La Laguna (ULL), E-38206 La Laguna, Tenerife, Spain
${ }^{6}$ Instituto de Fisíca, Universidad Nacional Autonoma de Mexico, Apdo. Postal 20-364, 01000, D.F, Mexico

${ }^{68}$ National Astronomy Observatories, Chinese Academy of Science, Beijing, 100012, China

${ }^{69}$ Department of Astronomy, Ohio State University, Columbus, OH 43210, USA

Received 2015 August 18; accepted 2015 December 6; published 2016 February 4

\begin{abstract}
In a six-year program started in 2014 July, the Extended Baryon Oscillation Spectroscopic Survey (eBOSS) will conduct novel cosmological observations using the BOSS spectrograph at Apache Point Observatory. These observations will be conducted simultaneously with the Time Domain Spectroscopic Survey (TDSS) designed for variability studies and the Spectroscopic Identification of eROSITA Sources (SPIDERS) program designed for studies of X-ray sources. In particular, eBOSS will measure with percent-level precision the distance-redshift relation with baryon acoustic oscillations (BAO) in the clustering of matter. eBOSS will use four different tracers of the underlying matter density field to vastly expand the volume covered by BOSS and map the large-scalestructures over the relatively unconstrained redshift range $0.6<z<2.2$. Using more than 250,000 new, spectroscopically confirmed luminous red galaxies at a median redshift $z=0.72$, we project that eBOSS will yield measurements of the angular diameter distance $d_{A}(z)$ to an accuracy of $1.2 \%$ and measurements of $H(z)$ to $2.1 \%$ when combined with the $z>0.6$ sample of BOSS galaxies. With $\sim 195,000$ new emission line galaxy redshifts, we expect BAO measurements of $d_{A}(z)$ to an accuracy of $3.1 \%$ and $H(z)$ to $4.7 \%$ at an effective redshift of $z=0.87$. A sample of more than 500,000 spectroscopically confirmed quasars will provide the first BAO distance measurements over the redshift range $0.9<z<2.2$, with expected precision of $2.8 \%$ and $4.2 \%$ on $d_{A}(z)$ and $H(z)$, respectively. Finally, with 60,000 new quasars and re-observation of 60,000 BOSS quasars, we will obtain new Ly $\alpha$ forest measurements at redshifts $z>2.1$; these new data will enhance the precision of $d_{A}(z)$ and $H(z)$ at $z>2.1$ by a factor of 1.44 relative to BOSS. Furthermore, eBOSS will provide improved tests of General Relativity on cosmological scales through redshift-space distortion measurements, improved tests for nonGaussianity in the primordial density field, and new constraints on the summed mass of all neutrino species. Here, we provide an overview of the cosmological goals, spectroscopic target sample, demonstration of spectral quality from early data, and projected cosmological constraints from eBOSS.
\end{abstract}

Key words: cosmology: observations - surveys

\footnotetext{
70 "la Caixa"-Severo Ochoa Scholar.

${ }^{71} \mathrm{SO}(\mathrm{IFT})$ Fellow.

72 NSF Astronomy \& Astrophysics, Postdoctoral Fellow.

${ }^{73}$ Hubble fellow.
} 


\section{INTRODUCTION}

The origin of the accelerating expansion of the universe is arguably the most important unknown in physics today and has inspired significant efforts to probe beyond the standard model of high-energy physics through observational cosmology. The recent measurements of the Cosmic Microwave Background (CMB) from the Planck satellite support a picture where the acceleration is driven by "dark energy" with density $\Omega_{\mathrm{de}}=0.692 \pm 0.012$ in a spatially flat universe (Planck Collaboration et al. 2015b). Combining these results with current measurements from baryon acoustic oscillations (BAO), SNe Ia, and $H_{0}$, the data imply a constant equation of state $w=-1.006_{-0.091}^{+0.085}$ at $95 \%$ confidence, where $w$ is the ratio of pressure to energy density for dark energy. Thus, current observations are generally consistent with the simplest picture where dark energy is described completely by Einstein's cosmological constant $(\Lambda)$.

New precise observations can unravel the origin of the accelerating universe; specifically, to determine if cosmic acceleration is caused by deviations in General Relativity (GR) on large scales or by a new form of (dark) energy. It is possible to decouple scenarios of acceleration that require dark energy from those that require modifications to GR by independently probing both cosmic expansion history and the structure growth rate. Four primary observational techniques are generally accepted as the most powerful toward obtaining that goal (e.g., Albrecht et al. 2006): SNe Ia, weak lensing, galaxy clusters, and BAO. Wide-field, optical spectroscopy figures prominently in three of these probes: spectroscopically observed galaxies improve calibration of photometric redshifts for weak lensing; direct spectroscopy of cluster galaxies provides precise redshifts and velocity dispersions as a proxy for cluster mass; and spectroscopy of galaxies and quasars provides the atlas of large-scale structure in which the BAO feature is embedded.

The Sloan Digital Sky Survey (SDSS; York et al. 2000) at Apache Point Observatory (APO) has consistently provided the largest spectroscopic samples for cosmological analysis. In the first two generations of SDSS, generally known as SDSS-I and -II, redshifts of nearly one million galaxies were measured spectroscopically (Abazajian et al. 2009). The Baryon Oscillation Spectroscopic Survey (BOSS; Dawson et al. 2013) performed spectroscopic observations of large-scale structure in SDSS-III (Eisenstein et al. 2011); BOSS recently completed spectroscopy on more than 1.5 million galaxies as faint as $i=19.9$ and more than 150,000 quasars as faint as $g=22$. Measurements of BAO with BOSS have led to $1 \%-2 \%$ precision measurements of the cosmological distance scale for redshifts $z<0.6$ and $z=2.5$.

With observations that commenced in 2014 July, SDSS-IV will continue this legacy in three distinct surveys. The second generation of the APO Galactic Evolution Experiment (APOGEE/APOGEE-2; Majewski et al. 2015) will investigate the formation and chemical history of the Milky Way using high-resolution, infrared spectroscopy of 300,000 stars. Mapping Nearby Galaxies at APO (MaNGA; Bundy et al. 2015) will measure the internal structure of approximately 10,000 galaxies using the BOSS spectrograph with cartridges outfitted for spatially resolved spectroscopy. The Extended Baryon Oscillation Spectroscopic Survey (eBOSS) is the new cosmological survey within SDSS-IV.
The eBOSS program will use the same 1000-fiber optical spectrographs as those in BOSS (Smee et al. 2013). We will expand the selection of luminous red galaxies (LRG) beyond that probed by BOSS and obtain better than a $1.0 \%$ precision distance estimate when combined with the $z>0.6$ tail of the BOSS galaxy population. With observations of a new sample of emission line galaxies (ELG) over the period 2016-2018, eBOSS will produce a $2.0 \%$ precision distance estimate at higher redshifts. We will obtain a $1.8 \%$ precision distance estimate in the redshift range $0.9<z<2.2$ using quasars that have luminosities and areal densities well-suited to sensitivity of the BOSS spectrographs. Finally, we will sharpen the BOSS Ly $\alpha$ forest measurements by a factor of 1.44 with a new selection of $z>2.1$ quasars, providing stronger leverage on the history of dark energy. Concurrent with the eBOSS survey, we will perform two complementary programs that use $\sim 100$ of the BOSS spectrograph fibers within each field of view. The Time Domain Spectroscopic Survey (TDSS; Morganson et al. 2015) will target variable stars and quasars. The Spectroscopic Identification of eROSITA Sources (SPIDERS) will target active galactic nuclei, quasars, X-ray emitting stars, and galaxy clusters identified in X-ray images of wide-area surveys carried out by ROSAT, XMM-Newton, and, eventually, eROSITA (Merloni et al. 2012).

With four classes of spectroscopic targets (LRG, ELG, quasar, Ly $\alpha$ forest quasar), eBOSS will enable the first high precision distance measurements in the epochs when dark energy emerged as the dominant dynamical component of the Universe. In addition to BAO distance measurements, eBOSS will provide new tests of GR on cosmological scales through redshift-space distortions (RSD), new tests for non-Gaussianity in the primordial density field, and new constraints on the summed mass of all neutrino species.

This paper is one of a series of technical papers describing the eBOSS survey. Details of the LRG target selection algorithm is described in Prakash et al. (2015b) while Myers et al. (2015) presents the quasar target selection algorithms. The ELG selection is still under investigation; Raichoor et al. (2015), Comparat et al. (2015a) and Delubac (2016, in preparation) present various approaches and results. Here, we provide the scientific motivation behind eBOSS, summarize the observation strategy for the cosmological tracers, present the expected quality of spectra for each target class, and compute the projected cosmological constraints. In Section 2, we provide a review of the cosmological signature of $\mathrm{BAO}$ and RSD in the matter power spectrum. We use BOSS measurements to demonstrate the sensitivity of spectroscopic surveys to both signatures and to demonstrate sources of systematic errors that must be addressed in eBOSS and future spectroscopic programs. We present the eBOSS program in Section 3, including time allocation, expected areal coverage, and requirements for target selection. In Section 4 we discuss the survey strategy, selection of the galaxies and quasars that will be used to measure the matter power spectrum, and statistics after fiber assignment. Favorable weather during SDSS-III led to an early completion of the BOSS program. A fraction of the remaining time was allocated to an eBOSS pilot program known as the Sloan Extended Quasar, ELG, and LRG Survey (SEQUELS). Using the results of those 66 dedicated plates, a demonstration of the data quality for each eBOSS target class is presented in Section 5. We summarize the expected quality of the data with respect to the low-level specifications required to 
achieve percent-level precision distance estimates in Section 6. We also present plans to improve the data reduction software in Section 6. Finally, the cosmological projections for eBOSS are provided in Section 7 and a summary is provided in Section 8 .

\section{SIGNATURE OF BAO AND RSD IN SPECTROSCOPIC SURVEYS}

Experience from BOSS led to the survey design for eBOSS described in Sections 3 and 4. BOSS proceeded with the primary goal of obtaining new measurements of the cosmic distance scale through BAO at redshifts $z<0.6$ and at $z=2.5$. While there have been other successful BAO programs (e.g., the $2 \mathrm{dF}$ Galaxy Redshift Survey, 6dF Galaxy Survey and WiggleZ Dark Energy Survey; Cole et al. 2005; Beutler et al. 2011; Blake et al. 2011b), we only review BOSS here because it provides a direct demonstration of spectroscopic constraints on cosmology that we expect from eBOSS, and sets the scene for this survey. In what follows, we outline the BOSS observational program in Section 2.1, the resulting BAO measurements in Section 2.2, and RSD measurements in Section 2.3.

\subsection{Sample of Galaxies and Quasars from BOSS}

BOSS is described in detail in Dawson et al. (2013), so we provide only a quick summary to highlight the essential features that are either inherent or complementary to the eBOSS program. BOSS and eBOSS use the same telescope as that used in SDSS-I and -II: the $2.5 \mathrm{~m}$ Sloan Foundation Telescope at APO in New Mexico (Gunn et al. 2006). The BOSS spectrographs (Smee et al. 2013) were built with smaller fibers, new improved detectors, higher throughput, and a wider wavelength range than the SDSS spectrographs previously used at APO. There are two spectrographs, each covering the wavelength range $361-1014 \mathrm{~nm}$. The instrument is fed by 1000 optical fibers (500 per spectrograph), each subtending $2^{\prime \prime}$ diameter on the sky.

The detection of BAO (Eisenstein et al. 2005) in the SDSS LRG sample (Eisenstein et al. 2001) motivated a similar selection of galaxies as the primary sample of spectroscopic targets for BOSS. At the beginning of BOSS observations, the techniques for analysis were already advanced to the stage that the collaboration could reasonably expect to perform BAO distance measurements at roughly $1 \%$ precision with the galaxy sample. In a more experimental program, more than 150,000 quasars at redshifts $z>2.1$ were selected to measure fluctuations in the matter density field as observed through the $\operatorname{Ly} \alpha$ forest. Contrary to the galaxy program, the Ly $\alpha$ forest program was created to make the first BAO measurements with a new tracer with an expectation that any detection would be made with somewhat lower significance than the galaxy measurement.

The BOSS survey obtained roughly $10,000 \mathrm{deg}^{2}$ of spectroscopic coverage over a five year period. At the completion of the main BOSS program, fibers were plugged into 2438 unique spectroscopic plates that each cover a circular field of view with $3^{\circ}$ diameter. All of the BOSS spectra became public in 2015 January with Data Release 12 (DR12; Alam et al. 2015a). These data include spectra and classification of 343,160 unique low redshift galaxies (denoted "LOWZ"; $z_{\text {median }}=0.32$ ), 862,735 unique medium redshift galaxies (denoted "CMASS"; $z_{\text {median }}=0.57$ ), and 181,605 quasars between $2.1<z<3.5$, of which 160,786 are not tagged as broad absorption line (BAL) quasars.

\subsection{Baryon Acoustic Oscillations}

While the overall shape of the power spectrum informs our cosmological model, the most robust cosmological measurements from spectroscopic surveys derive from the BAO feature in the clustering of matter. Sound waves that propagate in the opaque early universe imprint a characteristic scale in the clustering of matter, providing a "standard ruler" whose length can be computed using straightforward physics and cosmological parameters that are constrained by $\mathrm{CMB}$ observations. The characteristic BAO feature has a comoving scale (roughly $150 \mathrm{Mpc}$ ) set by the integrated sound speed between the end of inflation and the decoupling of photons around $z=1000$. The detection of the acoustic oscillation scale (Eisenstein et al. 2005 ) is one of the signature accomplishments of SDSS.

Measuring the angle subtended by the characteristic BAO feature at a given redshift provides a means to estimate the angular diameter distance, $d_{A}(z)$. Similarly, a measurement of the redshift interval over which the BAO feature extends provides a means to directly measure the Hubble parameter $H$ $(z)$ at that redshift. In general, there is a combination of $d_{A}(z)$ and $H(z)$ denoted by a generalized distance parameter " $R$ " that is better constrained than either of these two quantities alone. In the specific geometry of the measured clustering of BOSS galaxies, the best constrained "distance" is approximately $R \equiv D_{V}=\left[d_{A}^{2}(z) c z H^{-1}(z)\right]^{1 / 3}$ because there are two transverse dimensions and one line of sight dimension. In the Ly $\alpha$ forest, the enhancement of the radial signal gives greater weight to $H(z)$.

At low redshifts, BAO are a powerful complement to studies with SNe Ia: they have low systematic uncertainties; they measure distances in absolute units whereas supernovae only constrain relative distances; and they can directly measure both the cosmic expansion rate $H(z)$ and the distance-redshift relation $d_{A}(z)$. At high redshifts, the large comoving volume allows the BAO distance method to obtain remarkably precise measurements of the distance and expansion rate with better precision than SNe Ia. The BAO method is reviewed in detail by Weinberg et al. (2013, see their Section 4), including discussions of the underlying theory, the effects of nonlinear evolution and galaxy bias, survey design and statistical errors, control of systematics, recent observational results, and complementarity with other probes of cosmic acceleration.

\subsubsection{BOSS Constraints on BAO}

Using data from DR9, the first BAO measurement with the BOSS CMASS sample was published in 2012 (Anderson et al. 2012). The DR9 sample covered $1 / 3$ of the final BOSS volume, yet a clear BAO detection allowed a measurement of the distance to $z=0.57$ with a precision of $\sigma_{R}=1.7 \%$. The most recent galaxy clustering measurements with BOSS (Anderson et al. 2014b) produce a clear BAO detection using both the LOWZ and CMASS samples of DR11 galaxies. The BAO detection in the CMASS sample provides a $1.0 \%$ measurement of the generalized distance to $z=0.57$, the most precise distance constraint ever obtained from a galaxy survey. The LOWZ sample produces a measurement to $z=0.32$ with $2.1 \%$ precision. 
A thorough examination of potential systematics in the clustering of the BOSS CMASS galaxies revealed a $10 \%$ decrease in the detected number density of galaxies when comparing areas with high stellar density to those with low stellar density (Ross et al. 2012a). The correlation of galaxy density with stellar density is the most significant known bias on measured clustering, likely caused by incomplete deblending of detected objects in crowded fields of the SDSS imaging data. On the other hand, no significant correlation is seen between number density and potential imaging systematic errors in the LOWZ sample of galaxies. The lack of bias is likely because the lower redshift sample appears brighter and is less vulnerable to imaging artifacts such as the deblending problems seen in the fainter, high redshift sample. A weighting scheme based on galaxy surface brightness and stellar density was devised to reduce the systematic effect of stellar density on the measured clustering signal. When focusing on scales less than $150 \mathrm{Mpc}$, tests on mock catalogs reveal that the weights produce no significant bias on the mean measured correlation function. The results imply that systematic errors in galaxy clustering due to artifacts in imaging data (causing density fluctuations as high as 10\%) can be removed if those artifacts are identified.

The first measurement of clustering in the Ly $\alpha$ forest of BOSS quasars was reported in Slosar et al. (2011). In 2012, the first measurements of BAO using the Ly $\alpha$ forest detected in the spectra of $z>2.15$ quasars were released. Using 48,640 quasars in the redshift range $2.15<z<3.5$, Busca et al. (2013) detected a peak in the correlation function at a separation equal to $1.01 \pm 0.03$ times the distance expected for the BAO peak within a concordance $\Lambda$ CDM cosmology. Using an alternative analysis of the same quasar sample that included some peculiar quasars and larger wavelength coverage, Slosar et al. (2013) reported a 2\% measurement error on the distance. In the subsequent DR11 analysis (Delubac et al. 2015a), 137,562 DR11 quasars in the redshift range $2.1<z<3.5$ were used to detect a peak in the correlation function and constrain the distance to a precision of $2.1 \%$. Decomposing the optimized distance, the DR11 measurement yields a measurement $H(z)=222 \pm 7 \mathrm{~km} \mathrm{~s}^{-1} \mathrm{Mpc}^{-1}$ and $d_{A}(z)=1662 \pm 96 \mathrm{Mpc}$ at an effective redshift $z=2.34$.

Modeling the cosmic distance scale at $z>2$ can be enhanced beyond the Ly $\alpha$ forest auto-correlation measurement by adding a measurement of the cross-correlation of quasars with the Ly $\alpha$ forest absorption field. In particular, the relatively high bias of quasars provides leverage to improve precision in the measurement of the angular diameter distance relative to the Ly $\alpha$ forest auto-correlation. Font-Ribera et al. (2014a) use the DR11 sample of quasars to measure BAO in the Ly $\alpha$ forestquasar cross correlation both along the line of sight and across the line of sight. The best fit correlation function at an effective redshift $z_{e}=2.36$ translates to a measurement $H\left(z_{e}\right)=226 \pm 8 \mathrm{~km} \mathrm{~s}^{-1} \mathrm{Mpc}^{-1}$ and $d_{A}\left(z_{e}\right)=1590 \pm 60$ Mpc. Because the Ly $\alpha$ forest sightlines are effectively sampled at random, there is no evidence for systematic errors from imaging artifacts in the derived clustering of either Ly $\alpha$ forest auto-correlation or Ly $\alpha$ forest-quasar cross correlation.

In summary, BOSS provides the most precise BAO measurements to date using luminous galaxies and correlations in the Ly $\alpha$ forest from high redshift quasars. Highlighting the complementarity between BAO and $\mathrm{SNe}$ Ia, Aubourg et al. (2014) explore models with the combination of SNe Ia and BAO calibrated by the sound horizon scale as measured by Planck. They show that the combined Hubble Diagram (Betoule et al. 2014) of SNe Ia from the Supernova Legacy Survey (Conley et al. 2011) and SDSS-II Supernova Survey (Frieman et al. 2008; Sako et al. 2014) can be extrapolated to $z=0$ when anchored at intermediate redshift by BAO measurements. The $\mathrm{BAO}$ distance measurements effectively provide calibration of the absolute magnitude of SNe Ia and lead to a $1.7 \%$ precision measurement, $H_{0}=67.3 \pm 1.1 \mathrm{~km} \mathrm{~s}^{-1} \mathrm{Mpc}^{-1}$. This value agrees with the value of $H_{0}$ derived from $\mathrm{CMB}$ data alone under an assumption of flat $\Lambda \mathrm{CDM}$ but is in mild tension with Cepheid-derived distances; for example Riess et al. (2011) report $H_{0}=73.8 \pm 2.4 \mathrm{~km} \mathrm{~s}^{-1} \mathrm{Mpc}^{-1}$.

Extending the joint $\mathrm{CMB}, \mathrm{BAO}$, and $\mathrm{SNe}$ analysis to models of dark energy, Aubourg et al. (2014) report a measurement of the constant equation of state for dark energy $w=-0.98 \pm 0.06$ when curvature is treated as a free parameter. Allowing an additional free parameter to account for a possible time-evolving equation of state, the joint BAO$\mathrm{SNe}$ measurements produce only weak constraints on evolution: $w_{a}=-0.6 \pm 0.6$, where $w(a)=w_{0}+w_{a}(1-a)$ (e.g., Chevallier \& Polarski 2001; Linder 2003). As shown in Figure 1, SNe Ia provide only weak constraints on the Hubble Diagram over the redshift range $1<z<2$, and BOSS has no sensitivity to the cosmic interval that lies between the galaxy and the $\operatorname{Ly} \alpha$ forest samples. It is in this $0.6<z<2$ regime where the universe is expected to transition from matterdominated to dark energy-dominated. New measurements of the cosmic distance scale over this redshift range have the potential to improve constraints on models of a time-evolving equation of state for dark energy.

\subsection{Redshift Space Distortions and Modified Gravity}

Dark energy is often invoked to explain current CMB, SNe, and BAO observations that imply an accelerating universe. It is also possible to explain the accelerated expansion of the universe by modifying gravity at large scales. The galaxy redshifts used in spectroscopic BAO measurements of the expansion history help differentiate these two possible effects through measurements of the growth of structure via RSD (Kaiser 1987).

RSD arise because the gravitational pull of matter overdensities causes velocity deviations from the smooth Hubble flow expansion of the universe. These peculiar velocities are imprinted in galaxy redshift surveys in which recessional velocity is used as the line of sight coordinate for galaxy positions. Although the correlation function of galaxies is isotropic in real space, the peculiar velocities lead to an increase in the amplitude of radial clustering relative to transverse clustering when the correlation function is measured in redshift space. The resulting anisotropy in the clustering of galaxies is correlated with the speed at which structure grows; deviations from GR causing slower or faster growth give smaller or larger anisotropic distortions in the observed redshift-space clustering.

In general, the amplitude of clustering at a given redshift is parameterized by $\sigma_{8}(z)$, the rms fluctuations in spheres of radius $8 h^{-1} \mathrm{Mpc}$. The degree of anisotropy due to RSD depends on the rate of change of the amplitude of clustering. This change is typically parameterized as a function of the logarithm of the expansion scale parameter $f \sigma_{8}=\partial \sigma_{8} / \partial \ln a$, where $a=(1+z)^{-1}$ is the dimensionless cosmic expansion 


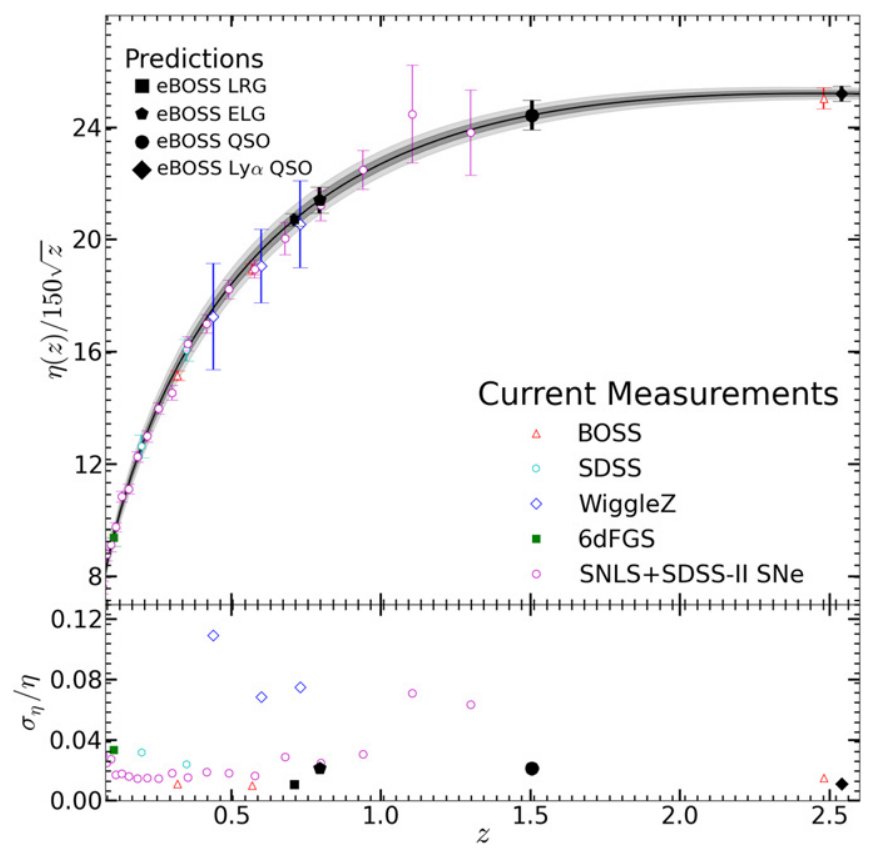

Figure 1. Projections for eBOSS LRG, ELG, and quasar distance measurements on a Hubble Diagram presented in comoving distance $(\eta)$ vs. redshift. Current BAO measurements from BOSS, SDSS (Xu et al. 2013; Ross et al. 2015), 6dF Galaxy Survey (6dFGS), and WiggleZ (Parkinson et al. 2012) are compared to SNe Ia measurements (Betoule et al. 2014) and Planck predictions (solid curve) obtained by marginalizing over the full likelihood function.

factor. Because RSD measurements are sensitive to the product of the growth rate and the amplitude of matter fluctuations, a wide range in redshift coverage is essential to constrain the evolution in clustering amplitude and directly probe gravity.

\subsubsection{BOSS Constraints on RSD and Modified Gravity}

Reid et al. (2012) and Samushia et al. (2013) presented the first measurements and cosmological interpretation of RSD in the BOSS DR9 galaxy sample. With these results, they constrain the parameter combination $f \sigma_{8}=0.43 \pm 0.07$. Using the larger DR11 sample, Samushia et al. (2014) constrain the parameter combination $f \sigma_{8}=0.447 \pm 0.028$ under an assumption of a spatially flat universe with cosmological constant. Using the DR10 sample to constrain RSD to smaller scales of 0.8-32 $h^{-1}$ Mpc, Reid et al. (2014) find a model-dependent constraint $f \sigma_{8}=0.450 \pm 0.011$ obtained in fitting a standard halo occupation distribution model to the anisotropic clustering. Other measurements from the same data have found similar results. A summary of current RSD measurements is presented in Figure 2.

Constraints on gravity from RSD become increasingly powerful as the measurements push to smaller scales, as evidenced by the higher precision measurements of Reid et al. (2014) relative to Samushia et al. (2014) and others. Cosmological measurements from small-scale clustering are dependent on the accuracy of the modeling on quasi-linear and nonlinear scales. The development and evaluation of analytic, phenomenological, and halo occupation models for anisotropic clustering remains a focus with the BOSS galaxy samples (e.g., Chuang et al. 2013; Beutler et al. 2014b; Guo et al. 2015). A study of several models in configuration-space tested against mock galaxy catalogs indicates that the clustering signal can be well characterized on scales in the range $40<s<80 h^{-1} \mathrm{Mpc}$

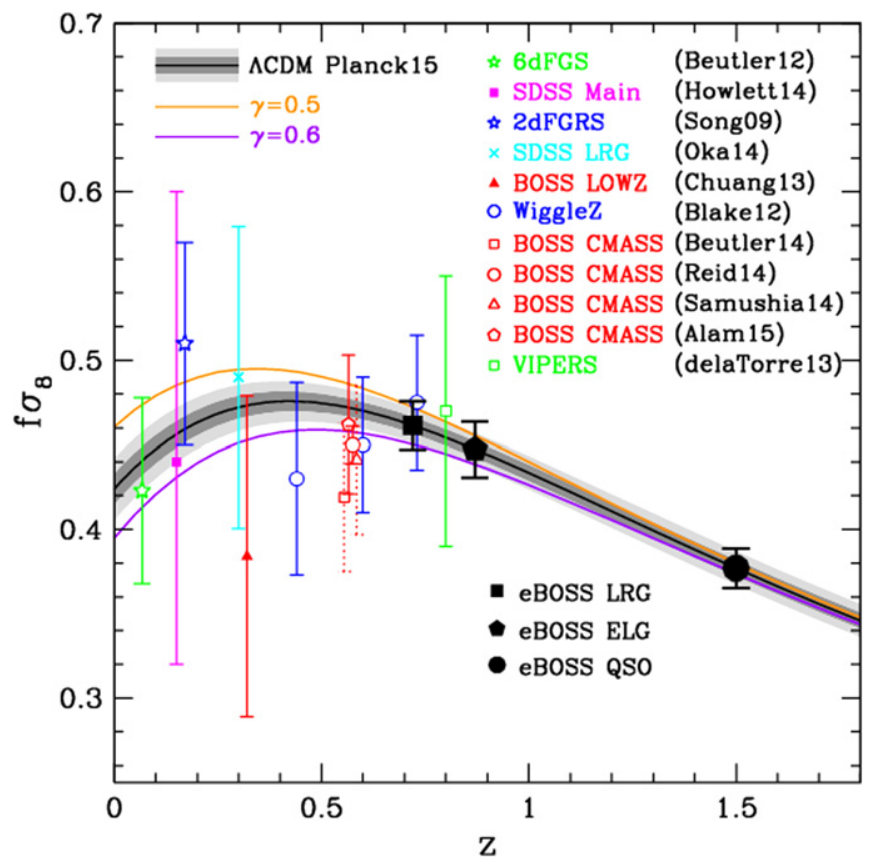

Figure 2. Current RSD constraints on the growth as a function of redshift compared to the projected measurements from eBOSS. The current measurements include those discussed in Section 2.3.1 and those for 6dFGS (Beutler et al. 2012), the main SDSS sample (Howlett et al. 2015), 2dFGRS (Song \& Percival 2009), the SDSS LRG sample (Oka et al. 2014), a recent result from the BOSS CMASS sample (Alam et al. 2015b), WiggleZ (Blake et al. 2012), and VIPERS (de la Torre et al. 2013). Various models of modified gravity are shown, each with the same background expansion, the same comoving BAO position, and amplitude of the power spectrum normalized to that of the CMB at high redshifts. The black curve shows the growth in a $\Lambda$ CDM universe, assuming the Planck best fit model parameters. The yellow curve shows $\gamma=0.5$ where $f=\Omega_{M}^{\gamma}$ (Linder 2005). The purple curve shows $\gamma=0.6$.

(White et al. 2015). Certain models, such as those based on Lagrangian perturbation theory, are able to fit the mock clustering samples without significant bias on scales above 25-30 $h^{-1}$ Mpc. Continued development of theoretical models that allow use of smaller scale data may tighten the current BOSS constraints still further.

\section{THE EXTENDED BARYON OSCILLATION SPECTROSCOPIC SURVEY}

As described in Section 2, BOSS completed a survey of $10,000 \mathrm{deg}^{2}$ and enabled the best BAO distance measurements to date at $z<0.6$ and the most precise distance measurements of any kind at $z \sim 2.5$. This success motivated the SDSS-IV collaboration to repurpose the BOSS spectrographs for eBOSS. The primary goal of eBOSS is to extend the BOSS galaxy measurements to $0.6<z<1$ and to make the first measurements of clustering with quasars as tracers and the first measurements of BAO at redshifts $1<z<2$. The strategy to make these measurements is not purely based on an optimized figure of merit. Instead, we follow a similar philosophy to BOSS and designed eBOSS to expand a well-understood sample while exploring new tracers over a fundamentally new redshift regime. We planned the expanded galaxy sample to achieve $1 \%$ precision on BAO measurements as in the BOSS galaxy sample and the new tracers to achieve $2 \%$ precision as in the BOSS Ly $\alpha$ sample.

Experience from BOSS led to the design of an LRG sample to measure clustering over redshifts $0.6<z<1.0$. As before, 
the BAO analysis tools for this type of galaxy are wellestablished. We designed the eBOSS LRG program to match the $1 \%$ precision on generalized distance achieved with the BOSS galaxy sample. We use the Fisher matrix formalism of Seo \& Eisenstein (2007) to determine BAO-based errors on the angular diameter distance and the Hubble parameter given an observing volume, number density and bias of galaxies. We assume a monotonically decreasing number density and a bias of $1.7 \sigma_{8}(0) / \sigma_{8}(z)$, approximately correct assuming the sample continues the evolution of the reddest galaxies observed in BOSS (Guo et al. 2013; Ross et al. 2014). We find that number density can be traded for areal coverage for number densities around $50 \mathrm{deg}^{-2}$ without degrading the precision on the BAO distance measurement. This relationship holds true for a fairly wide range of number densities, lending us flexibility in the design of the LRG program. Assuming a survey area on the order of thousands of square degrees, the balance of density and survey area leads to the general requirement that 300,000 LRG's in the redshift range $0.6<z<1.0$ are required to achieve $1 \%$ precision on the generalized distance.

Just as BOSS introduced the $\operatorname{Ly} \alpha$ forest sample as a new probe of clustering, eBOSS will introduce two new selections that will expand the legacy of SDSS. The first of these selections will be quasars in the redshift range $0.9<z<2.2$. Because quasars are bright and trace large-scale structure with a high bias, they are the most effective tool to constrain BAO at these redshifts. The lower bound on redshift is set to have slight overlap with the eBOSS galaxy samples while the upper bound is set to have slight overlap with the BOSS and eBOSS Ly $\alpha$ forest sample. The potential of this sample is limited by the intrinsic number density of the quasar population. In a series of spectroscopic observations with BOSS, 1877 quasars were classified over $14.5 \mathrm{deg}^{2}$ near the celestial equator. After correcting for targeting efficiency and selecting confirmed quasars at $0.9<z<2.2$ and $g<22$, the expected surface density of the quasar population is $82.6 \mathrm{deg}^{-2}$ (PalanqueDelabrouille et al. 2013a). We therefore are forced to design the quasar program with a lower volume density to sample largescale structure than the galaxy programs. We make BAO projections assuming a flat redshift distribution and the bias relationship $b(z)=0.53+0.29(1+z)^{2}$ determined by Croom et al. (2005) and consistent with SDSS measurements (Myers et al. 2007, 2006). Unlike the LRG targets, the limited surface density will lead to BAO measurements that are fundamentally limited by shot noise. In order to obtain a $2 \%$ measurement comparable to the BOSS Ly $\alpha$ sample, the quasars must sample the intrinsic population with high completeness over as much area as possible. We establish the maximal areal coverage in Section 3.1 and report the required quasar density there.

Potential risk in any one target class can be mitigated by observing a number of different galaxy types overlapping in redshift and using cross-correlation between different populations. The predominantly passive galaxies in the LRG sample are typically classified by absorption lines against faint continuum emission, leading to a practical limit that each target's $z$-band magnitude be brighter than roughly $z_{\mathrm{AB}} \sim 20$. The emission lines in blue galaxies with significant star formation facilitate spectroscopic confirmation at high redshifts with an optical spectrograph. Because ELG's are numerous and have emission line fluxes correlated with the observed $u$ or $g$ band magnitudes (Comparat et al. 2015b), they can be selected at a much higher density than the LRG sample. For these reasons, we identified an ELG sample over an area that ensures a BAO detection with minimal diversion from the LRG and quasar programs. We conservatively assume that the bias of the sample is $1.0 \sigma_{8}(0) / \sigma_{8}(z)$, slightly lower than expected (Comparat et al. 2013a, 2013b). As with the LRG sample, Fisher projections indicate that the shot noise and areal coverage contribute in such a manner that the BAO distance error remains relatively constant for a fixed number of objects for a sample with density of hundreds per square degree. We find that a $2 \%$ distance $\mathrm{BAO}$ measurement can be achieved with a total sample size of approximately 190,000 spectroscopically confirmed ELG's with a uniform redshift distribution in the redshift range $0.6<z<1.0$ or $0.7<z<1.1$.

In the DR11 BOSS sample, the BAO feature at an effective redshift $z=0.57$ was measured at a precision of $0.9 \%$ using CMASS galaxy targets at a density $85 \mathrm{deg}^{-2}$ (Anderson et al. 2014a). The BAO feature was measured at an effective redshift $z=2.34$ at a precision of $2 \%$ using $\operatorname{Ly} \alpha$ quasars at an average density $16 \mathrm{deg}^{-2}$ (Delubac et al. 2015a). The marginal value of each $\operatorname{Ly} \alpha$ quasar therefore exceeds that of a typical galaxy in constraining the matter power spectrum at BAO scales, as long as the Ly $\alpha$ targets can be reliably identified. We reserved as many fibers as possible for efficient detection and observations of $\operatorname{Ly} \alpha$ quasars for the purpose of improving the Ly $\alpha$ forest measurements obtained in BOSS.

Of equal importance to BAO are measurements of RSD with each of these samples. However, as discussed in Section 2.3, the current RSD measurement errors are primarily driven by theoretical uncertainties in the modeling rather than by the statistical power of the target samples.We therefore rely entirely on the high-level BAO goals for determining the observing strategy and properties of the target samples. The requirements for target densities are driven by the goal of obtaining $1 \%, 2 \%$, and $2 \%$ BAO distance measurements on the LRG, quasar, and ELG samples, respectively. We also present additional requirements on the uniformity of these samples intended to mitigate potential systematic errors in clustering measurements. In this section, we present the program that will meet these high-level objectives.

\subsection{The eBOSS Cosmology Program}

Over six years, eBOSS will provide the first percent-level distance measurements with BAO and RSD in the redshift range $0.6<z<2$, when cosmic expansion transitioned from deceleration to acceleration. Using LRG, quasars, ELG, and Ly $\alpha$ absorption as tracers of the underlying density field, eBOSS will probe the largest volume to date of any cosmological redshift survey. We designed a program of LRG, ELG, and quasar targets distributed over the available fibers with a balance that satisfies the high level goals of obtaining 1\%-2\% distance measurements while also meeting a series of practical constraints.

In BOSS, we typically finished observations of a spectroscopic plate in five 15 minute exposures with 20 minutes of overhead due to field acquisition, calibration exposures, and CCD readout. These exposures produced spectra of sufficient quality to classify the targets in the CMASS sample as faint as $i=19.9 \quad$ (AB) with over $98 \%$ completeness (Bolton et al. 2012). A series of pilot studies using dedicated plates in 2013 and 2014 proved that the same spectral depth is adequate to classify quasars from $0.9<z<2.2$, LRGs to 
$z<0.8$, and ELGs at $0.6<z<1.1$. Since a typical field can be completed in $1.5 \mathrm{hr}$ of exposure and overhead, we anticipate that 1800 plates can be completed over the roughly $5400 \mathrm{hr}$ allocated to eBOSS if the 50\% weather efficiency experienced during SDSS-III continues for SDSS-IV. Considering that each plate will contain 100 fibers dedicated to calibration targets and roughly 100 fibers dedicated to SPIDERS or TDSS targets, a total of $1.44 \mathrm{M}$ fibers will be available for targets designed to trace large-scale structure. Given this expected time allocation and potential sample size, we designed the program as follows.

1. First, we are constrained by the finite area of a spectroscopic plate $\left(7 \mathrm{deg}^{2}\right)$ and the requirement of a large-scale structure survey to obtain areal coverage with limited gaps between plates. As in previous SDSS programs, each eBOSS field center is assigned in the tiling process (Blanton et al. 2003). We chose a density of field centers to an average of one plate per $5.0 \mathrm{deg}^{2}$ to avoid gaps in coverage and mitigate inefficiency in fiber assignments caused by density fluctuations in the target sample. At this tiling density, 1800 plates can provide a total survey area up to $9000 \mathrm{deg}^{2}$. The TDSS and SPIDERS projects will each use an average of 50 fibers per plate and calibration targets require a minimum of 100 fibers per plate. With 800 remaining fibers per plate, the available density of cosmological tracers is $160 \mathrm{deg}^{-2}$. We use this density of fibers to shape the LRG and quasar samples which will be observed together over the majority of the program. We isolated the ELG targets to a series of dedicated plates. Those plates will contain almost entirely ELG targets except for a few additional objects at a maximum density of 10 fibers $\mathrm{deg}^{-2}$. To conduct the ELG program, we will reduce the maximal area of the LRG, quasar, and Ly $\alpha$ quasar samples from $9000 \mathrm{deg}^{2}$ to $7500 \mathrm{deg}^{2}$, thus leaving 300 plates for the ELG program. As discussed below and in Section 4, the density and areal coverage of those ELG targets will be decided based on final target selection algorithms and available imaging data. By doing so, we only reduce the volume of the primary LRG and quasar samples by $16 \%$ but obtain a sample of ELG targets that will provide a $\mathrm{BAO}$ constraint comparable to the DR9 BOSS galaxy results (Anderson et al. 2012) over a new redshift range.

2. At an area of $7500 \mathrm{deg}^{2}$, the Fisher projections predict that $0.9<z<2.2$ quasars at a density of $58 \mathrm{deg}^{-2}$ will produce a $2 \%$ distance measurement. Because the sample is shot-noise limited, any fractional increase in the number density translates to the same fractional improvement in the projected BAO uncertainty. Roughly $13 \mathrm{deg}^{-2}$ of $0.9<z<2.2$ quasars are already known from SDSS-I, -II, or -III, leaving only $45 \mathrm{deg}^{-2}$ quasars to be identified and spectroscopically confirmed in eBOSS. Because this sample probes a fundamentally new redshift range, we design the program primarily around the observations of quasars, allocating fibers at a density of $90 \mathrm{deg}^{-2}$ to ensure that we obtain the minimal target density. All objects assigned to the target class denoted QSO_CORE form the basis of this sample; the purity of this core sample needs to be at least $50 \%$ to meet the high-level goal of a $2 \%$ BAO distance measurement when combined with the sample of known quasars.
Throughout, purity is defined as the fraction of objects that are reliably classified in the redshift range of interest.

3. The LRG sample must contain at least 300,000 spectroscopically confirmed galaxies in the redshift range $0.6<z<1.0$ to obtain the high-level goal of a $1 \%$ BAO distance measurement. These objects will augment the tail of the BOSS CMASS sample in the range $0.6<z<0.7$ and increase the reach of the luminous galaxy sample to $z=1.0$. Over $7500 \mathrm{deg}^{2}$, the corresponding density of galaxies with a successful redshift in the desired redshift range is $40 \mathrm{deg}^{-2}$. In terms of projected BAO errors, a fractional change in the number density is equivalent to the same fractional change in area. In BOSS, luminous galaxies were reliably identified in imaging data and spectroscopically confirmed in a sample with purity that typically exceeded $95 \%$. The eBOSS sample requires higher absolute luminosity to maintain a reasonable rate of spectroscopic classification at the higher redshifts. However, the apparent magnitudes are still fainter and features are shifted further into the red, likely reducing redshift efficiency. Pilot programs and visual inspection during BOSS originally indicated that we could exceed $80 \%$ purity at $0.6<z<1.0$. We therefore require that the $40 \mathrm{deg}^{-2}$ LRG sample be reliably classified and at redshifts $0.6<z<1.0$ from observations of LRG candidates at a density $50 \mathrm{deg}^{-2}$. As will be shown in Sections 4.2 and 4.3, the purity of the final sample falls short of requirements, leading to the only failure we expect in meeting survey design.

4. Unlike galaxies, the information extracted from each $\operatorname{Ly} \alpha$ quasar scales with the $\mathrm{S} / \mathrm{N}$ of the spectra in the Lya forest. Before the beginning of BOSS, McDonald \& Eisenstein (2007) presented possible BAO constraints from the Ly $\alpha$ forest accounting for area, density of quasars, resolution, and $\mathrm{S} / \mathrm{N}$. As explained in detail in Section 5.3, McQuinn \& White (2011) find that the relative value of a quasar roughly doubles as the $\mathrm{S} / \mathrm{N}$ per $\AA$ increases from one to two, but that deeper spectra approach diminishing returns once the depth exceeds a $\mathrm{S}$ / $\mathrm{N}$ of three per $\AA$. To capitalize on the benefits of deeper spectra, we will re-observe $z>2.1$ quasars that obtained $0.75<\mathrm{S} / \mathrm{N}<3$ per $\AA$ in the Ly $\alpha$ forest in BOSS. Given that the exposure times in eBOSS will be comparable to those in BOSS, we expect the typical $\mathrm{S} / \mathrm{N}$ to increase by $40 \%$ when these quasars are observed a second time. The average density of this sample over the BOSS footprint is about $8 \mathrm{deg}^{-2}$.

5. After fiber allocation to the core quasar, LRG, and repeat Ly $\alpha$ forest targets, an average of 12 fibers $\mathrm{deg}^{-2}$ remain for additional Ly $\alpha$ forest quasar targets. In regions with multiple epochs of SDSS imaging, photometric variability was used to enhance the selection of Ly $\alpha$ quasars in BOSS (Palanque-Delabrouille et al. 2011). In eBOSS, we will use the same technique applied to multi-epoch imaging data from the Palomar Transient Factory (PTF; Law et al. 2009; Rau et al. 2009). PTF $R$-band imaging data with at least five epochs covers $90 \%$ of the available eBOSS footprint. We expect an efficiency of about $20 \%$ in the selection of $\operatorname{Ly} \alpha$ quasars from variability. Nominally, we would allocate 12 fibers $\mathrm{deg}^{-2}$ to this sample, but instead allow a target density of $20 \mathrm{deg}^{-2}$ in the PTF regions with an expectation of $3-4 \mathrm{deg}^{-2}$ new, 
confirmed Ly $\alpha$ forest quasars. We increase the density to account for reduced LRG and quasar sample sizes caused by "fiber collisions" between objects that lie too close to neighboring targets.

6. As described above, the ELG program is allocated 300 plates with a goal of obtaining spectroscopic classification of 190,000 galaxies, where the predicted BAO precision depends primarily on the total number of ELGs rather than the density or volume covered. There remains uncertainty in the final ELG program, as the exact selection method is still under investigation. The algorithm that preferentially selects galaxies with significant [O II] $\lambda 3727$ emission will likely be the one that is most efficient at obtaining this sample. Given this uncertainty, we require only that the sample cover a redshift interval $\Delta z=0.4$ to ensure a cosmologically interesting volume, lie at a median redshift that is higher than the LRG sample, and have an upper bound $z<1.1$ to avoid confusion in [O II] line identification in the wavelength region that is dominated by sky lines. These constraints effectively limit the sample of 190,000 galaxies to lie at either $0.6<z<1.0$ or $0.7<z<1.1$. If targets were to fill the fibers at the lowest density possible, a sample selected at a density $170 \mathrm{deg}^{-2}$ over $1500 \mathrm{deg}^{2}$ would meet the requirements if attained at a purity exceeding $74 \%$. Likewise, a sample at $340 \mathrm{deg}^{-2}$ over $750 \mathrm{deg}^{2}$ would meet the requirements at the same purity. As with the LRG sample, a fractional change in the number density has the same consequence for BAO precision as would the same fractional change in areal coverage.

In total, the final eBOSS spectroscopy will consist of: LRG targets $(0.6<z<1.0)$ at a density of $50 \mathrm{deg}^{-2}$ and desired purity exceeding $80 \%$, ELG targets at a somewhat higher redshift over 300 dedicated plates with a desired purity exceeding 74\%, "clustering" quasar targets (denoted QSO_$\mathrm{CORE})$ to directly trace large-scale structure $(0.9<z<2.2)$ at a density of $90 \mathrm{deg}^{-2}$ and desired purity exceeding $50 \%$, reobservations of faint BOSS Ly $\alpha$ quasars $(z>2.1)$ at a density of $8 \mathrm{deg}^{-2}$, and new Ly $\alpha$ quasar candidates identified by variability at an average density of $18 \mathrm{deg}^{-2}$.

\subsection{Target Selection Requirements}

We will only be able to meet the projected cosmological constraints if our final large-scale clustering measurements are limited by statistical errors. This requires stringent control of systematic errors that can modulate the data on large scales, such as the impact of stellar contamination and dust extinction on target selection efficiency, variations in seeing that alter target selection and redshift success, and so on. These systematics have already been extensively studied within BOSS (Ross et al. 2011, 2012a; Ho et al. 2012; Pullen \& Hirata 2013), and the greater volume and greater statistical power at large scales from eBOSS will place new demands on homogeneity of the target samples. These effects are important primarily for measurements (such as neutrino masses, the effective number of neutrino species, and inflation) that use the full shape of the galaxy power spectrum. BAO measurements are largely protected because they rely on a relatively sharp feature, but with the high precision of eBOSS, one must be careful to extract the BAO signature in a way that is immune to large-scale modulations.
Based on experience from BOSS, we apply a strict list of requirements to the selection of the LRG, ELG, and clustering quasar target classes to limit these systematic effects. Because Ly $\alpha$ quasars provide random sightlines to sample the foreground density field, we do not require uniformity in the Ly $\alpha$ quasar sample.

1. Statistical and systematic uncertainties in redshift estimates dampen the BAO feature in the radial direction. We require that the data reduction pipeline provide a combined precision and accuracy of $\sigma_{v}<300 \mathrm{~km} \mathrm{~s}^{-1} \mathrm{rms}$ at all redshifts, where $\sigma_{v}$ is defined as $c \sigma_{z} /(1+z)$. Increasing the tolerance in redshift estimates to $<540 \mathrm{~km} \mathrm{~s}^{-1} \mathrm{rms}$ would increase the projected BAO and RSD uncertainty by $10 \%$. The identifying features in galaxy spectra are typically well-characterized emission or absorption features with widths less than $300 \mathrm{~km} \mathrm{~s}^{-1}$. We expect to have redshift precision significantly better than this requirement for all classified galaxies; we expect occasional line confusion to introduce larger redshift errors that would be classified as "catastrophic," as addressed in the next item.

The prominent emission lines in quasars are broad and subject to velocity shifts with respect to the systemic redshift (e.g., Hewett \& Wild 2010; Richards et al. 2011). Measurements of clustering in the BOSS quasar sample imply redshift errors as large as $\Delta z /(1+z)=0.003$ (White et al. 2012). In addition to inflated redshift errors, Font-Ribera et al. (2013) provides compelling evidence for apparent velocity biases in the $z>2.1$ BOSS quasar sample of roughly $160 \mathrm{~km} \mathrm{~s}^{-1}$. Comparing automated redshifts to redshifts based on $\operatorname{Mg}$ II $\lambda 2799$, we believe we can improve the redshifting of quasars from those in BOSS and meet the strict redshift accuracy and precision requirements for quasars at $z<1.5$. To acknowledge the difficulty in redshift accuracy for the high redshift region of the clustering quasar sample, we relax the requirement to $[300+400(z-1.5)] \mathrm{km} \mathrm{s}^{-1}$ for objects at $z>1.5$. Any errors from the $\mathrm{Mg}$ II redshifts would contribute an additional degradation in the redshift accuracy. We estimate that degrading the redshift accuracy by $70 \%$ (by the same factor across all redshifts) increases the projected BAO and RSD uncertainty in the quasar sample by factor of 1.1 .

2. Redshift errors exceeding $1000 \mathrm{~km} \mathrm{~s}^{-1}$ can potentially bias the derived clustering signal by falsely projecting classified spectra onto the wrong redshift, possibly through line confusion or contamination by sky lines. As long as the fraction of objects with catastrophic redshift failures is small compared to the number of true tracers at any redshift, the misidentified fraction should lead to small enough changes in clustering to not bias the estimate of the BAO position. To minimize the impact of catastrophic errors, we require that the spectroscopic pipeline classify spectra with fewer than $1 \%$ catastrophic errors, where the redshifts are not known to be in error. As with the redshift accuracy requirement, we relax the definition of a catastrophic failure in the quasar sample to unknown errors in excess of $3000 \mathrm{~km} \mathrm{~s}^{-1}$.

3. The maximum absolute variation in expected galaxy density as a function of imaging survey sensitivity, stellar density, and Galactic extinction must be less than $15 \%$ (peak to peak). As discussed in Section 2.2, variations 
smaller than $10 \%$ have been mitigated in BOSS by straightforward weighting schemes. Based on this experience, we expect that we can extend galaxy weights to regions with $15 \%$ variation in target density with little effect on measurements in the power spectrum in the range $0.02 h \mathrm{Mpc}^{-1}<k<0.3 h \mathrm{Mpc}^{-1}$. Any areas with fluctuations greater than $15 \%$ could be discarded in the cosmology analysis and thus degrade the final precision of the clustering measurement.

4. Finally, for all samples used to directly constrain clustering, we require that our target selection procedures be robust against variations in the imaging zeropoints. We require that sample densities vary by less than $15 \%$ for the estimated uncertainties in zeropoint for the input imaging data in each bandpass. Unlike the previous requirement for maximum variation in density, this requirement can be computed directly by evaluating the derivative of target density with respect to an offset in photometry in a single band.

\section{SURVEY DESIGN}

Several developments since the beginning of BOSS allow us to identify the new samples of tracers for eBOSS cosmological measurements. First, the recent release of infrared sky maps from the Wide-field Infrared Survey Explorer (WISE; Wright et al. 2010) makes it possible to expand the selection of LRG targets beyond that probed by BOSS. Second, recent spectroscopic observations prove that we can select targets from imaging data and spectroscopically confirm star-forming galaxies with high efficiency from the $2.5 \mathrm{~m}$ Sloan Foundation Telescope (Comparat et al. 2013b). The galaxies with strong emission lines make it possible to further extend the galaxy redshift survey. Selection for these targets is not yet finalized and we plan to conduct the ELG observations in the third and fourth years of eBOSS. Third, spectroscopic observations reveal that quasars in the redshift range $0.9<z<2.2$ can be efficiently identified from spectroscopy (Palanque-Delabrouille et al. 2013a). Finally, improved photometric target selection and time-domain imaging data provide a dense sample of $z>2.1$ quasars to significantly sharpen the cosmology measurements from the Ly $\alpha$ forest relative to BOSS.

The procedures to select targets and complete a program of spectroscopy are well established from BOSS. We will filter the photometric catalogs, design the spectroscopic plates, and acquire the spectroscopic data in a nearly identical fashion to the procedures outlined in Dawson et al. (2013). However, there are changes to each step in the process, listed below.

\subsection{Imaging and Catalog Creation}

The baseline data set for eBOSS target selection is the wellunderstood photometry obtained from the SDSS imaging camera (Gunn et al. 1998) in ugriz filters (Fukugita et al. 1996). The median 5- $\sigma$ depth for photometric observations of point sources is $u=22.15, g=23.13, r=22.70, i=22.20$, $z=20.71$. Photometry for each filter $X$ is presented in pointspread function (PSF) magnitudes (denoted $X_{\mathrm{PSF}}$ ), fiber2 magnitudes $\left(X_{\text {fib2 }}\right)$ to represent the fiber aperture losses, model magnitudes $\left(X_{\text {model }}\right)$, or cmodel magnitudes $\left(X_{\text {cmodel }}\right)$, as discussed in online SDSS documentation. ${ }^{74}$ The SDSS photon

\footnotetext{
74 http://www.sdss.org/dr12/algorithms/magnitudes
}

counts and object detections used in eBOSS target selection algorithms stem from the DR9 public release (Ahn et al. 2012). The BOSS DR9 photometry is internally calibrated using the "ubercalibration" procedure described in Padmanabhan et al. (2008), with residual systematic errors of approximately $1.5 \%$ in $u$ and $1 \%$ in the other four bands. eBOSS has recalibrated the flat fields in all five filters and zero-points in the griz bands using a combination of PanSTARRS-1 (Kaiser et al. 2010) and SDSS stellar photometry. The residual systematics are reduced to $0.9 \%, 0.7 \%, 0.7 \%$ and $0.8 \%$ in the griz bands, respectively (Finkbeiner et al. 2015). In addition, some poorly constrained zero-points with errors exceeding $3 \%$ in the DR9 data are now significantly improved.

The photometric selection for the BOSS galaxy sample was based entirely on imaging from SDSS. Photometry from the UKIRT Infrared Deep Sky Survey (UKIDSS; Lawrence et al. 2007) and the Galaxy Evolution Explorer (GALEX; Martin et al. 2005) were used to enhance the selection of Ly $\alpha$ forest quasars (Bovy et al. 2012). The use of additional imaging resources to identify the primary cosmological sample set a precedent that we follow in eBOSS. The primary source of external imaging data will be the highly uniform, infrared (IR) photometry from the WISE satellite. The catalog of IR sources is derived using forced photometry informed by positions of sources in the SDSS imaging data. The full process is explained in Lang et al. (2014). The resulting 3.4 and $4.6 \mu \mathrm{m}$ (W1 and $W 2$, respectively) magnitudes can be found in the public release. $^{75}$

The imaging sample to be used for selection of ELG targets is still under investigation. ELG targets could be selected using SDSS griz and $U$-band imaging from the South Galactic Cap U-band Sky Survey (SCUSS) conducted at the $2.3 \mathrm{~m}$ Bok Telescope on Kitt Peak. The reductions of those two imaging samples are complete and photometric catalogs are available over the entire SGC area. ELG targets could also be selected from deeper $g r z$ imaging obtained from the Dark Energy Camera (DECam; Flaugher et al. 2012). The deeper imaging data allows more precise identification of strong emission-line galaxies in the redshift range of interest. We currently have catalogs based on preliminary data reductions of first year DECam observations. Through a large program approved for the Dark Energy Spectroscopic Instrument (DESI; Levi et al. 2013), we expect to cover the majority of the SGC with DECam in grz. Known as the DECam Legacy Survey $(\mathrm{DECaLS})^{76}$, the raw data from those observations are immediately public and the DESI team plans to publicly release full reductions of these data. However, observations are scheduled over 2014-2017 and it is not yet clear how much area will be available for selection of eBOSS targets on the timescales needed for SDSS-IV observations.

Finally, we use multi-epoch imaging data from the PTF (Law et al. 2009) and data from the Faint Images of the Radio Sky at Twenty-Centimeters (FIRST; Becker et al. 1995) program to increase the number of quasars. PTF covers roughly $90 \%$ of the potential eBOSS footprint at a depth that is sufficient to produce and characterize light curves. These data are made publicly available by the PTF team. ${ }^{77}$ The average field is observed over four years in $4560 \mathrm{~s}$ exposures. Light curves are constructed with a customized image processing

\footnotetext{
75 http://unwise.me/

76 http://legacysurvey.org/

77 http://irsa.ipac.caltech.edu/Missions/ptf.html
} 
pipeline that produces coadditions of all images acquired in a single year. The depth of a resulting epoch is typically comparable to the depth of SDSS photometry. Earlier SDSS photometry is used to anchor the light curves and provide a longer time baseline to measure variability. The catalog of sources from FIRST is used to identify potential quasars matched to SDSS optical counterparts. Sources are selected from the 2013 June 5 version of the FIRST point source catalog. ${ }^{78}$

For all imaging samples, magnitudes are corrected for Galactic extinction using the Schlegel et al. (1998) models of dust absorption. Galactic extinction coefficients have been updated as recommended in Schlafly \& Finkbeiner (2011). The extinction coefficients $R_{u}, R_{g}, R_{r}, R_{i}$, and $R_{z}$ are changed from the values used in BOSS $(5.155,3.793,2.751,2.086,1.479)$ to $(4.239,3.303,2.285,1.698,1.263)$, respectively. An extinction coefficient $R_{W 1}=0.184$ is used for the WISE $3.4 \mu \mathrm{m}$ band and $R_{W 2}=0.113$ is used for the $4.6 \mu \mathrm{m}$ band (Fitzpatrick 1999).

In BOSS, we used a series of imaging flags to remove possible contaminants from the target samples. The standard CALIB_STATUS SDSS imaging flags (SATUR etc.) become increasingly poor at distinguishing real objects from artifacts at fainter magnitudes. We therefore make no explicit cuts on our galaxy or quasar targets based on the standard SDSS imaging flags. We do remove quasar targets with IMAGE_STATUS flags identified as "BAD_ROTATOR," "BAD_ASTROM," "BAD_FOCUS," "SHUTTERS," "FF_PETALS," "DEAD_CCD," or "NOISY_CCD." We rely on our use of multi-wavelength photometry (such as imaging from WISE) to identify true astrophysical objects as candidate sources for spectroscopy. We expect that some objects close to bright stars might have contaminated colors, causing spurious objects to enter into the target selection. Areas close to bright stars represent a tiny fraction of the sky and it is not obvious a priori how close to bright stars the photometry remains reliable, so we do not explicitly remove targets. Clustering analyses will likely either mask or re-weight the galaxy and quasar densities due to the loss of objects near bright stars, as has been done for the BOSS clustering analyses (e.g., Anderson et al. 2012).

\subsection{Target Selection Algorithms}

The targets for eBOSS spectroscopy will consist of: LRGs at $0.6<z<1.0$, ELGs at somewhat higher redshift, "clustering" quasars to directly trace large-scale structure $(0.9<z<2.2)$, re-observations of faint BOSS Ly $\alpha$ quasars $(z>2.1)$, and new Ly $\alpha$ quasars $(z>2.1)$. The selection algorithms for identifying the LRG and quasar samples are complete and observations of these targets have begun. The selection algorithm for ELG sample is under investigation. Here, we summarize the selection scheme and statistics of each sample.

\subsubsection{LRG Samples}

A full investigation of the LRG selection from imaging data is presented in the companion paper (Prakash et al. 2015b). The final sample was designed to extend the BOSS galaxy sample to higher redshifts following initial studies of an optical and infrared selection of LRGs (Prakash et al. 2015a). LRG candidates will be observed (assigned a fiber) at an average density of $50 \mathrm{deg}^{-2}$ as governed by the survey design

\footnotetext{
78 http://sundog.stsci.edu/first/catalogs/readme_13jun05.html
}

explained in Section 3.1. Targets are selected at a density to oversubscribe the fiber budget and ensure a high overall efficiency of fibers assigned to cosmological tracers. Given the fiber assignment statistics described in Section 4.3, we find that a parent sample with density of $60 \mathrm{deg}^{-2}$ is adequate to satisfy the requirement to spectroscopically observe objects at a density of $50 \mathrm{deg}^{-2}$.

At the redshifts of the LRG sample $(z>0.6)$, the $4000 \AA$ break is shifted into the SDSS $i$ filter, increasing the error of $u g r$ photometric estimates. In addition, the degradation of photometry at higher redshifts provides motivation to include ancillary photometric information beyond SDSS to improve the selection efficiency. The WISE W1 filter centered at 3.4 microns provides restframe coverage of the " 1.6 micron bump" that results from a reduction in the opacity of $\mathrm{H}^{-}$ions exhibited by old stellar populations (John 1988). The final LRG selection algorithm is tuned to identifying objects that are red in $r-i$, $i-z$ and $r-W 1$ colors as demonstrated in Prakash et al. (2015b).

As will be explained in Section 5.1, the estimate of the redshift distribution stems from visual inspections that are difficult to translate to the final pipeline performance. For that reason, we report maximum and minimum number densities that correspond to more or less optimistic interpretations of the visual inspections. Between $68.3 \%$ and $71.8 \%$ of the targets identified by this selection lie between $0.6<z<1.0$, depending on which level of confidence is assumed in the visual inspection process. The surface density of the sample is shown as a function of redshift in Table 1. Objects with spectra that fail to produce a reliable classification are labeled "Poor Spectra."

The density of M-stars and of galaxies at $0.5<z<0.6$ are both higher than what was expected following the initial pilot programs in BOSS. Because of these two contaminants, the parent population represented by the SDSS/WISE selection fails to meet the goal of $80 \%$ purity for galaxies in the redshift range $0.6<z<1.0$. Roughly $24 \%$ of the CMASS galaxies in BOSS lie at redshifts $z>0.6$ and roughly $4 \%$ lie at redshifts $z>0.7$. While not originally intended, those CMASS galaxies can be combined with the new eBOSS LRG galaxies to increase the overall sample size and obtain a $1 \%$ precision distance measurement.

We assess uniformity of the target sample through a regression analysis of surface density against tracers of potential systematics. We focus on systematics associated with imaging data and astrophysical effects such as dust extinction and stellar density. The resulting regression fits reveal that $92 \%$ of the potential imaging area in eBOSS has predicted surface density that varies by less than $15 \%$, thus satisfying the imaging uniformity requirements outlined in Section 3.2. The $8 \%$ of the area that fails to meet this requirement must be reexamined when spectroscopic observations are complete to assess variations after identification of contaminating sources such as stars. Likewise, we assess the variations in number density associated with errors in the zeropoint calibration. We find that zeropoint errors of $0.01 \mathrm{mag}$ in the rizW1 filters cause fractional changes in the number density of LRG targets of $2.26 \%, 2.5 \%, 6.24 \%$, and $0.6 \%$, respectively. Likely due to the fact that it is used for both a color boundary and a magnitude boundary, the target selection is most sensitive to the uncertainty in the $z$-band calibration; non-uniformity with $15 \%$ peak-to-peak amplitude occurs in regions where the $z$ - 
Table 1

Expected Redshift Distribution for the LRG, Quasar, and Ly $\alpha$ Quasar Samples

\begin{tabular}{|c|c|c|c|c|c|c|c|}
\hline & $\begin{array}{l}\text { CMASS } \\
\text { Known }\end{array}$ & $\begin{array}{c}\text { LRG } \\
\left(z_{\text {conf }}>0\right)^{\mathrm{a}}\end{array}$ & $\begin{array}{c}\text { LRG } \\
\left(z_{\text {conf }}>1\right)^{\mathrm{a}}\end{array}$ & $\begin{array}{c}\text { QSO_CORE } \\
\text { New }\end{array}$ & $\begin{array}{c}\text { QSO_CORE } \\
\text { Known }^{\text {b }}\end{array}$ & $\begin{array}{l}\text { Ly } \alpha \text { Quasar } \\
\text { Reobservation }\end{array}$ & $\begin{array}{l}\text { Quasar } \\
\text { PTF }^{\mathrm{c}}\end{array}$ \\
\hline Poor Spectra & $\cdots$ & 4.0 & 6.7 & 3.2 & 0.9 & 0 & 0.7 \\
\hline Stellar & $\cdots$ & 5.3 & 5.3 & 2.8 & 0.3 & 0 & 10.7 \\
\hline Galaxy & $\ldots$ & $\ldots$ & $\ldots$ & 6.6 & 0.4 & 0 & 1.5 \\
\hline $0.0<z<0.5$ & 27.3 & 0.6 & 0.6 & 1.0 & 0.4 & 0 & 0.2 \\
\hline $0.5<z<0.6$ & 45.7 & 6.2 & 5.9 & 1.1 & 0.4 & 0 & 0.1 \\
\hline $0.6<z<0.7$ & 19.4 & 15.2 & 14.8 & 1.4 & 0.7 & 0 & 0.2 \\
\hline $0.7<z<0.8$ & 3.5 & 15.3 & 14.7 & 1.4 & 1.3 & 0 & 0.2 \\
\hline $0.8<z<0.9$ & 0.2 & 9.4 & 8.7 & 2.2 & 1.5 & 0 & 0.2 \\
\hline $0.9<z<1.0$ & 0.03 & 3.2 & 2.7 & 3.6 & 1.0 & 0 & 0.3 \\
\hline $1.0<z<1.2$ & 0 & 0.6 & 0.5 & 8.4 & 1.8 & 0 & 0.5 \\
\hline $1.2<z<1.4$ & 0 & 0 & 0 & 10.3 & 1.8 & 0 & 0.4 \\
\hline $1.4<z<1.6$ & 0 & 0 & 0 & 10.3 & 2.1 & 0 & 0.6 \\
\hline $1.6<z<1.8$ & 0 & 0 & 0 & 9.9 & 2.0 & 0 & 0.4 \\
\hline $1.8<z<2.0$ & 0 & 0 & 0 & 9.2 & 1.9 & 0 & 0.3 \\
\hline $2.0<z<2.1$ & 0 & 0 & 0 & 4.0 & 1.0 & 0 & 0.2 \\
\hline $2.1<z<2.2$ & 0 & 0 & 0 & $2.2^{\mathrm{d}}$ & $1.6^{\mathrm{d}}$ & 0.5 & 0.2 \\
\hline $2.2<z<2.4$ & 0 & 0 & 0 & $1.8^{\mathrm{d}}$ & $4.5^{\mathrm{d}}$ & 2.9 & 0.5 \\
\hline $2.4<z<2.6$ & 0 & 0 & 0 & $1.1^{\mathrm{d}}$ & $3.1^{\mathrm{d}}$ & 1.9 & 0.5 \\
\hline $2.6<z<2.8$ & 0 & 0 & 0 & $0.7^{\mathrm{d}}$ & $1.4^{\mathrm{d}}$ & 1.0 & 0.6 \\
\hline $2.8<z<3.0$ & 0 & 0 & 0 & $0.3^{\mathrm{d}}$ & $0.8^{\mathrm{d}}$ & 0.7 & 0.5 \\
\hline $3.0<z<3.5$ & 0 & 0 & 0 & $0.4^{\mathrm{d}}$ & $1.2^{\mathrm{d}}$ & 0.9 & 0.5 \\
\hline$z>3.5$ & 0 & 0 & 0 & $0.1^{\mathrm{d}}$ & $0.1^{\mathrm{d}}$ & 0.3 & 0.2 \\
\hline Total Targets & 23 & 60 & 60 & 81.8 & 30.4 & 8.3 & 20 \\
\hline Total Tracers & 23.1 & 43.1 & 41.0 & $\mathbf{5 7 . 9}$ & 13.1 & 8.3 & 3.2 \\
\hline
\end{tabular}

Notes. The surface densities are presented in units of $\mathrm{deg}^{-2}$ assuming that $100 \%$ of the objects in the parent sample are spectroscopically observed. Entries highlighted in bold font denote the fraction of the sample that satisfies the high-level requirement for the redshift distribution of the sample.

${ }^{a}$ The redshift distribution for the LRG sample is determined by visual inspection. It is not clear how well the automated pipeline will perform in the final analysis, so we include the results from visual inspections with $z_{\text {conf }}>0$ in the first entry as the more optimistic estimate and results with $z_{\text {conf }}>1$ in the second entry as the less optimistic estimate.

${ }^{\mathrm{b}}$ Objects that satisfy the QSO_CORE selection that have reliable spectra from previous incarnations of SDSS will not be assigned fibers in eBOSS.

${ }^{c}$ The density of PTF-selected quasars is only computed over areas where targets are selected. Roughly $10 \%$ of the eBOSS footprint will not have PTF-selected quasar targets.

d The population of $z>2.1$ quasars that appear in the target selection for the clustering sample will be used for Ly $\alpha$ forest studies.

band zeropoint is in error by \pm 0.012 mag. Assuming a Gaussian dispersion of $0.008 \mathrm{mag}$ in the $z$-band calibration as discussed in Section 4.1 and in Finkbeiner et al. (2015), 13.3\% of the eBOSS volume exceeds the $15 \%$ uniformity requirement. This effect is not expected to be correlated with imaging systematics and in the worst case scenario, will have to be addressed through an independent census of zeropoints in each SDSS field. Analysis through mock catalogs will inform the manner in which zeropoint uncertainties are modeled in the clustering measurements. The full methodology of the uniformity tests is presented in Prakash et al. (2015b).

\subsubsection{Quasar Samples}

The algorithm to identify quasars in BOSS was intended to build a sample of $z>2.1$ quasars to map the large scale distribution of neutral hydrogen via absorption in the $\operatorname{Ly} \alpha$ forest. As explained in Ross et al. (2012b), the most uniform quasar sample for BOSS was identified from a density of 20 targets $\mathrm{deg}^{-2}$ using the "Extreme Deconvolution" (XDQSO; Bovy et al. 2011a, 2011b) selection. This selection was performed after estimating the relative density of stars and quasars as a function of color, magnitude, and photometric uncertainty. The probability that an object is a quasar is determined by the fraction of objects with similar photometric properties expected to be a quasar and not a star.
Following that precedent, the XDQSO algorithm will be again used to identify quasars for the QSO_CORE sample in eBOSS, but using the XDQSOz version of the algorithm (Bovy et al. 2012) that can be applied to select quasars in any redshift range.

To improve the completeness of the sample, objects with much lower XDQSO probabilities will be included in eBOSS relative to BOSS. The inclusion of lower probability objects will also tap into the population of $z>2.1$ quasars that were missed in BOSS and enhance the sample for Ly $\alpha$ forest clustering measurements.

Colors between optical and WISE passbands can be used to distinguish quasars from stellar objects characterized by blackbody spectra (for example Stern et al. 2012). Morphology selection helps reduce the number of lower luminosity, extended active galactic nucleus (AGN) sources in favor of compact, point-like quasars which map onto higher mass halos. In these ways, WISE imaging will be used to decrease stellar contamination and increase the overall efficiency of fiber assignments.

The final selection algorithm for quasars in the QSO_CORE sample is described in the companion paper on quasar target selection (Myers et al. 2015). The final selection when applying XDQSOz and the SDSS/WISE colors results in a target density of $115 \mathrm{deg}^{-2}$. The average density of targets falls to roughly 
$90 \mathrm{deg}^{-2}$ after removing objects that were confidently classified as a star, galaxy, or quasar in SDSS or BOSS spectra.

As shown in Table 1, pilot studies in BOSS indicate that the selection identifies 71 quasars $\mathrm{deg}^{-2}$ over $0.9<z<2.2$, of which 13 will already be known from previous observations. The selection will also increase the number of Ly $\alpha$ quasars: 6.6 new $z>2.1$ quasars $\mathrm{deg}^{-2}$ are expected. In addition to stars, we list the rate of galaxy contamination in the quasar sample; roughly $8 \%$ of the new targets turn out to be galaxies with significant line emission. If targeted at $100 \%$ completeness, this shot noise-limited sample would exceed the goal of $58 \mathrm{deg}^{-2}$, leading to potential improvements of up to $20 \%$ over the highlevel goal of a $2 \%$ precision distance measurement with this sample.

Performing the same regression analysis as on the LRG sample, we find that $\sim 90 \%$ of the eBOSS area satisfies the requirement of $<15 \%$ peak-to-peak variation in the quasar target density. The quasar target selection is very robust against zeropoint errors. We find that zeropoint errors of $0.01 \mathrm{mag}$ in any of the ugrizW1W2 filters cause fluctuations in target density of less than $1 \%$. The largest contributor to density fluctuations is the $g$-band calibration; a $0.01 \mathrm{mag}$ error leads to a $0.86 \%$ change in target density. Given the expected rms error in flux calibration is only 0.009 mag, we expect an rms scatter in target density of only $0.77 \%$ due to calibration errors. Only a negligible fraction of sky will see density fluctuations as large as $15 \%$.

Quasars selected for Ly $\alpha$ forest studies are not subject to the same strict requirements of uniformity as those used in direct clustering work. For this reason, a series of selections was used to increase the total number of $z>2.1$ quasars. The first selection was based on known quasars from BOSS. A known quasar is included in the eBOSS selection if it obtained a low signal-to-noise in the BOSS observation. The mean signal-tonoise ratio is computed over the range $1040<\lambda<1200 \AA$. Objects that did not have BALs identified in visual inspection and that have $0.75<\mathrm{S} / \mathrm{N}<3$ or $\mathrm{S} / \mathrm{N}=0$ are observed again in eBOSS. As explained in Myers et al. (2015) and in Table 1, we also identify unique new Ly $\alpha$ forest quasars in the PTF data at a density of $3.2 \mathrm{deg}^{-2}$ where PTF imaging is available to generate sufficient light curves. At redshifts $z>2.5$, the PTF selection identifies quasars at a density $2.7 \mathrm{deg}^{-2}$, of which $2.3 \mathrm{deg}^{-2}$ are unique to variability selection. Finally, a small number of objects from the FIRST catalogs are included as possible quasars. Because the target density is only around $1 \mathrm{deg}^{-2}$, we do not include the statistics from that sample in Table 1 .

\subsubsection{ELG Sample}

The ELG survey will begin in Fall 2016, the third year of eBOSS observations. By starting the ELG program two years later than the LRG and quasar programs, the eBOSS team has given itself time to perform deeper analysis of the potential selection algorithms. We conducted a series of observations in Fall 2014 to test possible techniques for the selection of ELG targets. At the time of writing, the tests are being used to evaluate the redshift success rates, redshift distributions, and rates of stellar contamination from four different selection algorithms.

Each of these four options relies on selecting targets from a subset of imaging data selected from SCUSS $U$-band, SDSS griz, WISE W1 and W2, or grz imaging with DECam. The tests
Table 2

Expected Redshift Distribution for the Four Potential ELG Selection Algorithms

\begin{tabular}{lcccc}
\hline \hline & & & DECam & \\
& gri + Uri & $\begin{array}{c}\text { Fisher Dis- } \\
\text { criminant }\end{array}$ & $\begin{array}{c}\text { (Low } \\
\text { Density) }\end{array}$ & $\begin{array}{c}\text { DECam } \\
\text { (High } \\
\text { Density) }\end{array}$ \\
\hline Poor Spectra & 64.7 & 19.8 & 17.1 & 24.1 \\
Stellar & 4.0 & 1.5 & 0.0 & 0.0 \\
$0.0<z<0.1$ & 2.1 & 2.9 & 0.6 & 0.6 \\
$0.1<z<0.2$ & 2.7 & 2.0 & 1.7 & 2.4 \\
$0.2<z<0.3$ & 3.6 & 2.5 & 1.6 & 2.0 \\
$0.3<z<0.4$ & 4.1 & 1.7 & 1.0 & 1.2 \\
$0.4<z<0.5$ & 4.8 & 2.8 & 0.6 & 0.7 \\
$0.5<z<0.6$ & 9.4 & 7.2 & 1.2 & 1.7 \\
$0.6<z<0.7$ & $\mathbf{2 7 . 6}$ & $\mathbf{2 5 . 4}$ & 3.3 & 3.7 \\
$0.7<z<0.8$ & $\mathbf{4 2 . 8}$ & $\mathbf{4 6 . 2}$ & $\mathbf{4 0 . 7}$ & $\mathbf{4 4 . 1}$ \\
$0.8<z<0.9$ & $\mathbf{2 5 . 9}$ & $\mathbf{4 0 . 4}$ & $\mathbf{6 5 . 3}$ & $\mathbf{7 4 . 1}$ \\
$0.9<z<1.0$ & $\mathbf{1 0 . 8}$ & $\mathbf{1 7 . 0}$ & $\mathbf{3 0 . 9}$ & $\mathbf{4 3 . 7}$ \\
$1.0<z<1.1$ & 2.5 & 6.5 & $\mathbf{1 1 . 1}$ & $\mathbf{1 6 . 9}$ \\
$1.1<z<1.2$ & 0.4 & 2.6 & 5.1 & 7.7 \\
$z>1.2$ & 2.2 & 2.9 & 9.5 & 16.9 \\
\hline Total Targets & 203.9 & 182 & 190.4 & 241.3 \\
Total Tracers & $\mathbf{1 0 7 . 1}$ & $\mathbf{1 2 9 . 0}$ & $\mathbf{1 4 8 . 0}$ & $\mathbf{1 7 8 . 8}$ \\
\hline & & & &
\end{tabular}

Notes. The surface densities are presented in units of $\mathrm{deg}^{-2}$ assuming that $100 \%$ of the objects in the parent sample are spectroscopically observed. Entries highlighted in bold font denote the fraction of the sample that satisfies the high-level requirement for the redshift distribution of the sample.

${ }^{\mathrm{a}}$ The desired range for DECam-selected ELGs is $0.7<z<1.1$ instead of $0.6<z<1.0$.

will also inform the final choice for imaging data to be used in the ELG selection. The expected redshift distribution for each candidate selection is presented in Table 2.

The first candidate for ELG selection uses SCUSS imaging for deeper $U$-band photometry to complement SDSS gri photometry. Objects for the spectroscopic sample are taken from the union of a $g-r$ and $r-i$ selection in SDSS imaging and a $U-r$ and $r-i$ selection in SCUSS and SDSS imaging. In addition to several cuts designed to reject point sources and ensure good photometry, objects satisfying $g-r<0.8$ and $r-i>0.8$ are included in the SDSS gri color selection. These cuts lead to a mean target density of $132.5 \mathrm{deg}^{-2}$. Objects satisfying $i-U>-3.5 *(r-i)+0.7$ and $r-i>0.7$ are included in the SCUSS and SDSS Uri selection at a density of $84.0 \mathrm{deg}^{-2}$. In all cases, photometry is assumed in extinctioncorrected model magnitudes. The selections are described in detail in Comparat et al. (2015a). The combined algorithm allows the selection of 180 objects per square degree over any region of the South Galactic Cap. The density in the test region was somewhat higher and had median redshift $z=0.72$. Many objects in this selection lie near the SDSS detection limit; photometric errors are sometimes as large as $0.5 \mathrm{mag}$. The large errors reduce the precision of the color estimates and effectively broaden the redshift distribution. Only $52.5 \%$ of the targets produce reliable redshift estimates in the redshift range intended for clustering studies. In addition, the large photometric errors allow fainter objects into the selection, thus reducing the bias relative to a sample represented by a more precisely defined magnitude limit.

The second candidate selection relies on the addition of the WISE photometry to the SDSS and SCUSS photometry. This scheme imposes a cut on Fisher discriminant quantities (Fisher 
1936) instead of cuts in color-color diagrams. In this case, the Fisher discriminant quantity is a linear combination of colors taken from UgrizW1. The exact definitions of the linear combination of colors, the training from a spectroscopic sample, and the expected results are described in Raichoor et al. (2015). The selection algorithm can be used to identify targets up to a density of $200 \mathrm{deg}^{-2}$, somewhat higher than the density in the test region. The test observations demonstrate a significant improvement over the SDSS+SCUSS $(g r i+U r i)$ selection. Roughly $71 \%$ of these objects are expected to produce reliable redshifts in the range $0.6<z<1.0$. The initial tests of this selection approach the requirement of $74 \%$ purity presented in Section 3.1.

The final two candidates for ELG selection use DECam $g r z$ photometry instead of SDSS, SCUSS, or WISE photometry. Both of these selections exceed the $74 \%$ purity requirement as detailed in Comparat et al. (2015a). The advantage of these selection schemes is that deeper DECam photometry allows more precise selection of star-forming galaxies at somewhat higher redshifts than the SDSS and SCUSS selections. For this reason, we increase the required redshift range for the spectroscopically confirmed tracer population from $0.6<z<1.0$ to $0.7<z<1.1$. The disadvantage of this selection scheme is that it relies on a relatively new imaging camera and a reduction pipeline that is in a state of development. The two candidate algorithms were tuned from spectroscopic observations of a broad color selection that produced targets to $g<22.8$ at a density of $597 \mathrm{deg}^{-2}$ in the test region. The Dark Energy Survey (DES) imaging is reduced using magnitudes determined in a $4^{\prime \prime}$ diameter aperture and in photometry similar to model magnitudes from SDSS. Extinction-corrected magnitudes are used throughout. Both selection algorithms apply the following cuts to reduce stellar contamination to nearly zero: $g_{\mathrm{APER}, 4}-g_{\text {DETMODEL }}<2$, $r_{\mathrm{APER}, 4}-r_{\mathrm{DETMODEL}}<2, \quad$ and $\quad z_{\mathrm{APER}, 4}-z_{\mathrm{DETMODEL}}<2$, where $X_{\mathrm{APER}, 4}$ is derived from an aperture two arcseconds in diameter and $X_{\text {DETMODEL }}$ is a DES measurement determined from SExtractor (Bertin \& Arnouts 1996) similar to modelmag in SDSS. The point source rejection also limits the number of quasars entering into the sample; the expected quasar contamination is expected to be less than $1 \%$ at $z<1.1$.

The first of the DECam selections (denoted "Low Density") was tuned to achieve a target density of roughly $190 \mathrm{deg}^{-2}$. An equivalent to model magnitudes is used in the selection. The color cuts that define that sample are as follows:
1. $21.6<g<22.8$
2. $0.3<g-r<0.7$
3. $0.25<r-z<1.4$
4. $r-z>0.45 *(g-r)+0.4$
5. $r-z<0.7 *(g-r)+0.8$.

The resulting redshift distribution is shown in the fourth column of Table $2.77 .8 \pm 1.1 \%$ of objects lie at $0.7<z<1.1$ with a median redshift $z=0.86$.

The second of the DECam selections was tuned to achieve a target density of roughly $240 \mathrm{deg}^{-2}$. The color cuts that define that sample are as follows:
1. $21.5<g<22.8$
2. $0.2<g-r<0.7$
3. $0.25<r-z<1.4$
4. $r-z>0.45 *(g-r)+0.4$
5. $r-z<0.8 *(g-r)+1$

The redshift distribution from this "High Density" DECam selection is shown in the last column of Table $2.74 .1 \pm 0.5 \%$ of objects lie at $0.7<z<1.1$ with a median redshift $z=0.87$.

While it is possible to increase the density above $240 \mathrm{deg}^{-2}$ using DECam data, initial tests reveal that contamination from galaxies outside the desired redshift range reduces the efficiency of the selection. In addition, as the selection approaches $300 \mathrm{deg}^{-2}$, the density peaks around $3 \times 10^{-4} h^{3} \mathrm{Mpc}^{-3}$. At this density, BAO measurements become dominated by sample variance rather than shot noise and observing time is more efficiently spent expanding the survey volume than by increasing the density. When comparing the low and high density DECam selections presented above, one must also consider the strength of the [O II] and [O III] emission line fluxes. The weighted mean of the [O III] line flux is 6.6 and $6.9 \times 10^{-17} \mathrm{erg} \mathrm{cm}^{2} \mathrm{~s}^{-1}$ for the low and high density selections, respectively. Likewise, the [O II] line flux is 8.15 and $8.5 \times 10^{-17} \mathrm{erg} \mathrm{cm}^{2} \mathrm{~s}^{-1}$. Although the high density selection produces a somewhat smaller rate of galaxies at $0.7<z<1.1$, the typical line strengths are $5 \%$ higher than in the low density selection, thus making it more robust to automated classification.

Tests of uniformity, sensitivity to zeropoint uncertainty, and average target density are underway (Delubac 2016, in preparation). A final decision on the selection to be used for eBOSS is expected in early 2016. The sample that is able to produce a uniform target density, redshift classification exceeding $74 \%$ efficiency over the appropriate redshift range, and imaging area sufficient for a total sample of 300 plates will be chosen. If more than one selection meets these requirements, the selection algorithms producing the highest median redshift will be the one used for the ELG sample.

\subsection{Tiling and Fiber Assignment}

The goal of survey tiling is to create a spatial distribution of tiles that maximizes the number of targets observed with the minimum number of tiles. We define the tiling completeness as the fraction of objects in a given class that were assigned fibers. We refer to the fiber efficiency as the fraction of available fibers used for unique science targets. In BOSS, the quantity of highest priority was the tiling completeness for "decollided" targets. The physical size of the ferrules that support each fiber in the plug plate limits the proximity of neighboring targets to $62^{\prime \prime}$. Groups of targets that lie within $62^{\prime \prime}$ of one another are denoted "collision groups." The decollided set contains all targets that are not within collision groups, combined with the subset of collided targets that can be assigned fibers on a single plate. A collision pair contributes one galaxy to the decollided set because, in all cases, one target from the pair will be assigned a fiber. If the pair is located within a region observed by more than one tile, the second object may be assigned a fiber as well. Thus the completeness of the collided set will be non-zero.

The spatial distribution of tiles in BOSS was set such that the decollided completeness of galaxy and Ly $\alpha$ forest quasar samples was $100 \%$. Due to the inhomogeneity of the target list, it is not possible to reach $100 \%$ decollided completeness and $100 \%$ fiber efficiency. Since the tiling completeness was the higher priority, the BOSS fiber efficiency for LOWZ, CMASS, and quasar targets was $\sim 90 \%$.

In eBOSS, we adopted a tiered-priority system for survey targets to maximize both tiling completeness and fiber 


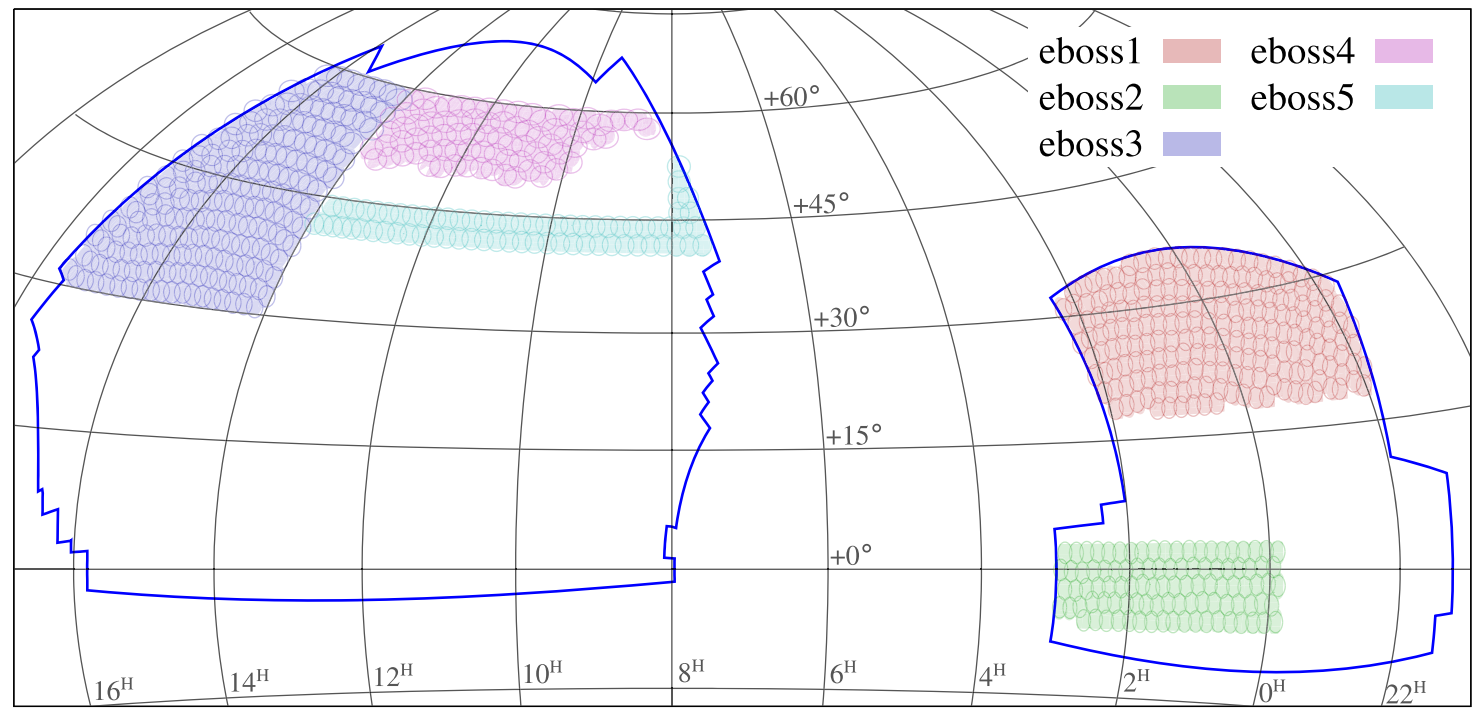

Figure 3. Field centers for eboss [1-5]. The SEQUELS area is clearly defined by white space between the boundaries of eboss 4 and eboss5. The area covered here is the area that was tiled in the beginning of SDSS-IV and the approximate survey area expected to be completed in the first two years of observation.

Table 3

Fiber Assignment Statistics in the First Five Tiled Regions For the Quasar Programs

\begin{tabular}{|c|c|c|c|c|c|c|c|c|}
\hline Chunk & $\begin{array}{c}\text { Number of } \\
\text { Plates }\end{array}$ & $\begin{array}{l}\text { Area } \\
\left(\operatorname{deg}^{2}\right)\end{array}$ & $\begin{array}{l}\text { Total CORE }^{\mathrm{a}} \\
\text { Target Density }\end{array}$ & $\begin{array}{l}\text { New CORE } \\
\text { Target Density }\end{array}$ & $\begin{array}{l}\text { New CORE } \\
\text { Fiber Density }\end{array}$ & $\begin{array}{l}\text { Reobserved Ly } \alpha \\
\text { Fiber Density }\end{array}$ & $\begin{array}{l}\text { PTF }^{\mathrm{c}} \text { Target } \\
\text { Density }\end{array}$ & $\begin{array}{c}\text { PTF }^{\mathrm{c}} \text { Fiber } \\
\text { Density }\end{array}$ \\
\hline eboss1 & 199 & 987.9 & 100.9 & 84.10 & 80.47 & 6.19 & 18.59 & 17.23 \\
\hline eboss2 & 98 & 469.4 & 113.1 & 80.84 & 77.08 & 9.47 & 10.56 & 9.67 \\
\hline eboss 4 & 80 & 397.1 & 114.4 & 73.47 & 69.86 & 9.16 & 28.70 & 26.36 \\
\hline eboss 5 & 70 & 353.7 & 116.4 & 84.19 & 79.68 & 8.49 & 14.51 & 13.30 \\
\hline
\end{tabular}

Notes. Surface densities are all presented in units of $\mathrm{deg}^{-2}$. The "Target Density" corresponds to the density of objects from each class that satisfy the photometric selection Criteria. The "Fiber Density" corresponds to the density of targets that were assigned fibers in the tiling process.

"The sample denoted "Total CORE" corresponds to all targets satisfying the QSO_CORE selection criteria, regardless of prior observations.

b The sample denoted "New CORE" corresponds to all targets satisfying the QSO_CORE selection criteria that were not observed previously and are candidates for fiber assignment in eBOSS.

" The sample denoted "PTF" corresponds to all targets satisfying the variability selection criteria using PTF imaging data that are exclusive of all other samples.

efficiency. All non-LRG targets receive maximal priority and are assigned fibers first. The tiling solution must satisfy the requirement of $100 \%$ tiling completeness for the decollided set of all non-LRG targets. For LRGs, we no longer require full decollided completeness. Rather, the density of LRG targets intentionally oversubscribes the remaining fiber budget. The input sample is tiled at a lower density due to collisions with higher-priority targets, collisions between multiple LRGs, and lack of available fibers for LRGs in the decollided set. We refer to collisions of LRGs with higher-priority targets as knockouts in order to differentiate them with collisions between two LRGs. To achieve the survey goal of candidate LRG targets observed at $50 \mathrm{deg}^{-2}$, the input target catalog is increased to a target density of $60 \mathrm{deg}^{-2}$. Thus, after high-priority targets are assigned fibers, plates that have a surplus of unused fibers can sometimes be used to observe LRGs at a density higher than $50 \mathrm{deg}^{-2}$. The quantity of interest for LRG tiling is the completeness of targets that are both decollided (i.e., with respect to other LRGs) and no-knockout (i.e., with respect to high-priority targets). The former effect can be corrected in clustering measurements by proper weighting of tiled targets. The latter is essentially a mask overlayed on the LRG portion of the survey. In eBOSS, we require that the completeness of decollided, no-knockout LRGs be at least $95 \%$. This layered tiling scheme allows $8 \%$ more area to be covered than would be possible if the eBOSS fiber efficiency were the same as in BOSS.

In the first round of fiber assignments-the non-LRG targets -we must specify the priority for fiber assignments within collision groups. Because the quasar targets are significantly higher density than TDSS and SPIDERS targets, quasarTDSS/SPIDERS collisions are fractionally more common for TDSS/SPIDERS target classes. Thus collisions are resolved in the following order (highest to lowest priority): SPIDERS, TDSS, reobservation of known quasars, clustering quasars, and variability-selected quasars. Quasars found in the FIRST survey and white dwarf stars that can be used as possible calibration standards are given the final priorities for resolving fiber collisions. Because the density of clustering quasar targets is comparable to the galaxy sample in BOSS, and because the fiber assignments require 100\% completeness on the decollided sample, the resulting sample of clustering quasars follows tiling statistics that are very similar to the galaxies in BOSS.

Five distinct regions of sky (denoted ebossN, where $N$ is a number ranging from 1 to 5 ) were tiled in the first year of eBOSS. The area and tile centers for those regions are shown in Figure 3. These regions convey the average tiling statistics that can be expected for the quasar samples over the duration of the 
Table 4

Fiber Assignment Statistics in The First Five Regions Tiled for eBOSS

\begin{tabular}{cccccc}
\hline \hline Chunk & $\begin{array}{c}\text { LRG } \\
\text { Target } \\
\text { Density }\end{array}$ & $\begin{array}{c}\text { LRG } \\
\text { Knockout } \\
\text { Density }\end{array}$ & $\begin{array}{c}\text { Noknock, } \\
\text { Collided } \\
\text { Density }\end{array}$ & $\begin{array}{c}\text { LRG Fiber } \\
\text { Density }\end{array}$ & $\begin{array}{c}\text { Noknock, } \\
\text { Decollided } \\
\text { Completeness }\end{array}$ \\
\hline eboss1 & 64.66 & 4.41 & 3.56 & 56.64 & $98.9 \%$ \\
eboss2 & 58.73 & 3.87 & 2.53 & 52.90 & $99.7 \%$ \\
eboss3 & 60.53 & 4.11 & 2.75 & 52.74 & $97.3 \%$ \\
eboss4 & 57.12 & 4.07 & 2.48 & 49.24 & $96.6 \%$ \\
eboss5 & 59.37 & 4.81 & 2.66 & 50.37 & $96.4 \%$ \\
\hline
\end{tabular}

Note. Surface densities are all presented in units of $\mathrm{deg}^{-2}$.

program. The statistics of the quasar samples for each region are presented in Table 3. The input target density for the QSO_CORE sample of quasars ranges between 73 and $88 \mathrm{deg}^{-2}$, a bit lower than the expected density of $90 \mathrm{deg}^{-2}$. The density of previously observed objects that satisfies the primary quasar selection is $17,32,31,41$, and $32 \mathrm{deg}^{-2}$ for eboss [1-5], respectively. Region eboss 1 is outside the final SDSS-II footprint, so fewer SDSS spectra exist and the number of known targets is reduced by a factor of two. Generally, the variation in the density of known objects explains the variation in the new quasar sample; the total QSO_CORE density only ranges from 113.1 to $118.2 \mathrm{deg}^{-2}$ for eboss [2-5]. The total QSO_CORE density in eboss 1 is $100.9 \mathrm{deg}^{-2}$, significantly lower than the average of the other chunks. Regression tests indicate that this suppression in surface density is likely due to the higher extinction in eboss1 relative to the rest of the SDSS imaging area. See Myers et al. (2015) for a full discussion of non-uniformity in the quasar target selection.

On average, the input target sample of clustering quasars is assigned fibers at a completeness of $95 \%$, thus reducing the expected number density of $0.9<z<2.2$ quasars by $5 \%$ relative to the parent sample presented in Table 1. A similar trend is found for the PTF-selected quasars, but at slightly lower completeness $(\sim 92 \%)$ due to their lower priority in resolving fiber collisions. Note that the repeated observations of Ly $\alpha$ quasars are by definition exclusive of the QSO_CORE selection but the PTF-selected quasars do have significant overlap with the QSO_CORE sample. In Tables 1 and 3, we have assigned PTF-selected targets only to the QSO_CORE sample when overlap occurs. The PTF-selected densities presented in the table therefore reflect the density of unique PTF-selected targets. Because the FIRST sample only produces quasars at $1 \mathrm{deg}^{-2}$, we do not include the statistics of that sample in Table 3.

The area of the first five regions that is covered by only a single tile is $2054 \mathrm{deg}^{2}, 64 \%$ of the total $3193 \mathrm{deg}^{2}$ currently tiled. In these areas, there is no way to capture objects lost to fiber collisions with other targets. The remaining $1139 \mathrm{deg}^{2}$ is covered by two or more tiles. In these regions, the completeness of the collided objects increases significantly, leading to a total completeness on the quasar sample of close to $100 \%$.

In the second round of tiling, LRGs are the only target species and require no prioritization to resolve fiber collisions. The statistics of the LRG sample are presented in Table 4. Because the redshift distribution of the LRG sample only overlaps $\sim 8.5 \mathrm{deg}^{-2}$ new QSO_CORE objects at redshifts $z<1$, the masked region is mostly uncorrelated with clustering in the LRG sample. There is negligible overlap between the
LRG and TDSS/SPIDERS samples. To first order, the areas of sky restricted from observing LRG targets can be treated in a similar manner to regions lost to bright stars or imaging artifacts in BOSS. Quantifying the full consequences of the overlapping samples will be done on mock catalogs as part of future clustering analyses.

On average, the total completeness of the LRG sample is $87 \%$ while the completeness of decollided, noknock (noknockout) targets is $98 \%$. The average density of LRG targets given a fiber is $52 \mathrm{deg}^{-2}$, slightly exceeding the goal of $50 \mathrm{deg}^{-2}$. The resulting fiber assignments occasionally produce local fluctuations that may have insufficient completeness for clustering analysis. An example of the fluctuations of the decollided, noknock LRG targets tiled in eboss 3 is shown in the left hand panel of Figure 4. The area-weighted cumulative histogram of completeness over the entire region covered by the first five regions is shown in the right hand panel of Figure 4. Assuming these five regions are representative of the completeness we expect in the full eBOSS footprint, $5 \%$ of the area will fall below $85 \%$ completeness in the decollided, noknock LRG targets. These areas exceed the $15 \%$ uniformity requirement that we have generally assumed and may be excised from the clustering analysis depending on the results of future tests on mock catalogs.

In the 300-plate ELG program, all ELG targets will be assigned equal priority and ranked ahead of other targets. Because they have not been tiled over a cosmologically useful volume, we do not present the statistics of the ELG sample. Instead, those statistics will be included in a future paper dedicated to the final ELG target selection algorithm.

\subsection{Plate Design}

In the tiling stage described above, we assign science targets to fibers based on the input from target selection algorithms and the available fiber budget. In plate design, we convert those positions from celestial coordinates to the coordinate system of the telescope focal plane. We also assign additional targets to the reserved fibers to provide reference for sky subtraction and flux calibration.

Tests in BOSS reveal a measurable increase in skysubtraction residuals when the number of sky fibers drops below 80 per plate. We therefore maintain the density of at least 80 sky fibers per plate in eBOSS. In addition, we now require that at least 30 sky fibers are assigned to each spectrograph.

Standard stars for flux calibration in BOSS were selected at a density of 20 per plate over the magnitude range $15<r_{\text {fib } 2}<19$. We maintain a density of 20 F-stars selected as standard stars, but now require that at least six stars be assigned to both spectrographs. The F-stars at the faint limit in BOSS produced spectra that were of marginal quality for flux calibration, while the brightest stars had nearly three orders of magnitude higher flux than the faintest science targets and increase the risk of contamination from cross-talk between neighboring fibers. For this reason, we choose eBOSS F-stars to have a narrower magnitude distribution, with $16<r_{\text {fib2 }}<18$. To compensate for the reduced density in candidate F-stars, we increase the scaled distance in extinctioncorrected color space from the color of a fiducial F star by a factor of two relative to BOSS. We now require that 

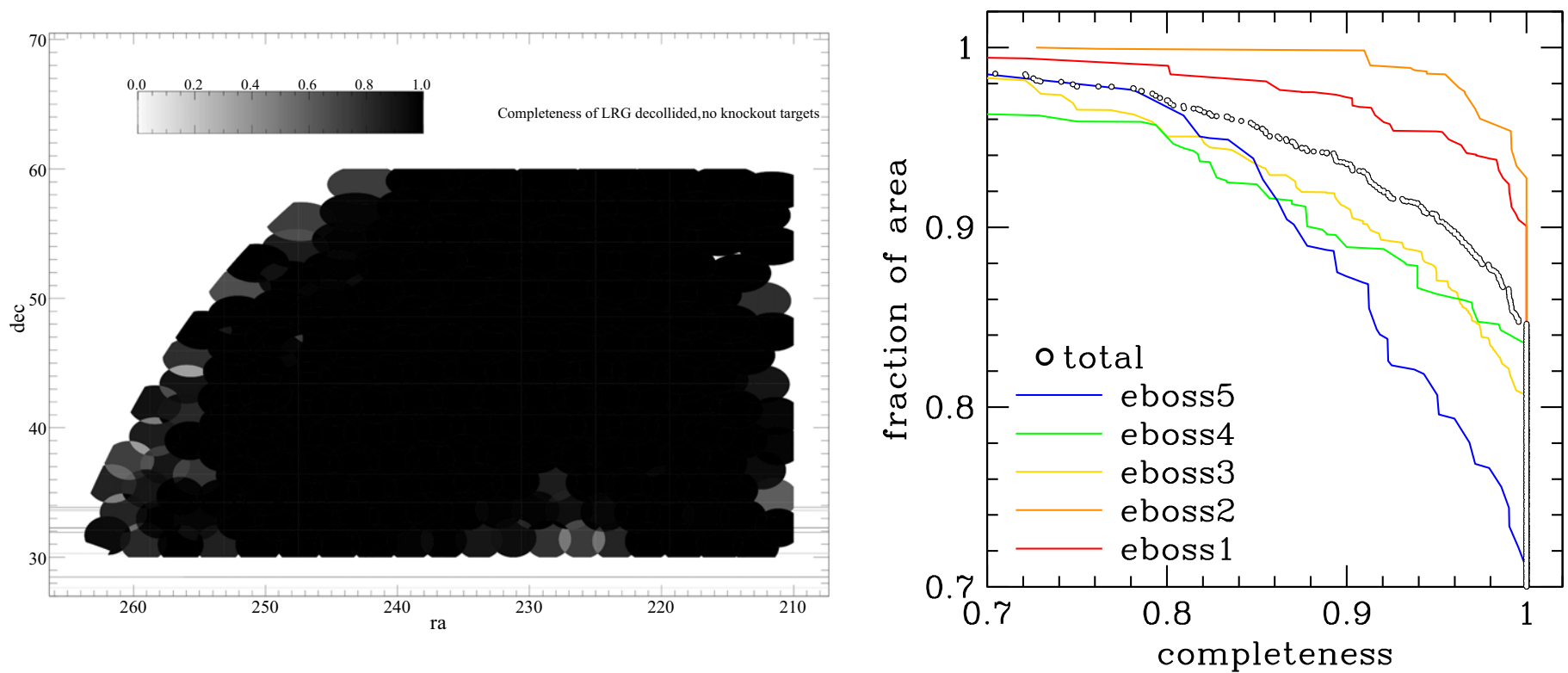

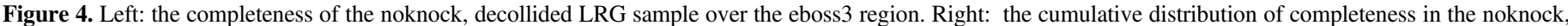
decollided LRG sample. The distribution is weighted by the area of each independent sector defined by areas covered by overlapping and unique tiles.

$m_{\text {dist }}<0.16$, where

$$
\begin{aligned}
m_{\mathrm{dist}}= & {\left[((u-g)-0.82)^{2}+((g-r)-0.30)^{2}\right.} \\
& \left.+((r-i)-0.09)^{2}+((i-z)-0.02)^{2}\right]^{1 / 2} .
\end{aligned}
$$

In BOSS, after accounting for predicted atmospheric differential refraction (ADR) for each plate, galaxy targets were centered in the focal plane to maximize throughput for $5400 \AA$ light. The hole positions for quasar targets were centered to maximize throughput at $4000 \AA$ to increase the $\mathrm{S} / \mathrm{N}$ in the Ly $\alpha$ forest. In eBOSS, the QSO_CORE sample is selected to lie at $z>0.9$ to provide direct clustering measurements while the other quasar samples are selected to lie at $z>2.1$ to increase the $\operatorname{Ly} \alpha$ forest signal with respect to BOSS. All objects selected in the QSO_CORE sample are centered in the focal plane at a position corresponding to the focus of $5400 \AA$ light. These targets will have appropriate flux calibration derived from the F-stars. All $z>2.1$ quasars known from BOSS and all quasar candidates selected by variability are centered in the focal plane at a position corresponding to the focus of $4000 \AA$ light. While not appropriate for flux calibration in the current data reduction pipeline, the routines developed in Margala et al. (2015) can be applied to the Ly $\alpha$ target spectra to improve the broadband distortion introduced by the ADR offset. As before, the wavelength that determines the center of the hole position is recorded in the quantity LAMBDA_EFF. In BOSS, washers manufactured with an adhesive were applied to the back of the plates at the location of quasar targets. Washer thickness varied according to distance from the plate center to account for the $0-300 \mu \mathrm{m}$ difference in focus between $4000 \AA$ light and $5400 \AA$ light. These washers matched the quasar fibers to the focal plane for $4000 \AA$ light for optimal focus by offsetting the fiber tip in the direction perpendicular to the surface of the plate. However, due to weather, guiding, and other stochastic processes, we were unable to confirm the ability of the sticky washers to significantly improve the $\mathrm{S} / \mathrm{N}$ in the $\operatorname{Ly} \alpha$ forest. Those washers are therefore not applied in eBOSS.

\subsection{Observing Sequence}

Simple data reductions are performed in real time to provide quick feedback to the observers and to track the depth of each exposure. The observers acquire signal on each field until the accumulated depth exceeds an empirically derived threshold for each camera. In BOSS, the depth was tuned to reach desired redshift success rates on the highest redshift galaxy targets in the shortest exposure time possible. Exposures were tuned so that the typical square of the signal-to-noise per pixel was at least 22 for an object with native SDSS magnitude $i_{\mathrm{fib} 2}=21$ over the wavelengths covered by the $i$-band filter. We also required the square of the signal-to-noise per pixel to be at least 10 for an object with $g_{\text {fib2 }}=22$ over the wavelengths covered by the $g$-band filter. In eBOSS, we will maintain the same thresholds for the beginning of the survey. To ensure that we define plate completion criteria so that we complete the goal of 1800 plates, we will use the first year of observation to empirically determine new exposure thresholds. As was done in BOSS, the depths will be tuned to ensure that we can reliably classify targets in the shortest exposure possible. It is likely that different thresholds will be used for the quasar and LRG plates than those used for the ELG plates.

Because quasars and LRGs are the primary focus of the eBOSS program, the majority of fibers and survey area are dedicated to these two target classes. The total area covered by these samples will be $7500 \mathrm{deg}^{2}$ divided over one contiguous region in the SGC and one contiguous region in the NGC. The eboss 1 region presented in Figure 3 was tiled to cover an SGC area at high declination that is easily observed. The eboss 2 region was chosen to obtain spectroscopy in a $500 \mathrm{deg}^{2}$ region that overlaps with the DES footprint. The NGC is tiled in one contiguous region at declination below $60^{\circ}$.

As was explained in Section 4.2, the sample selection for the quasar and LRG samples is complete. The first two years of observation will be dedicated almost exclusively to these primary fields. After two years, approximately 600 plates should be completed for the LRG and quasar targets, producing a sample comparable in area to the DR9 CMASS cosmology 
sample. Under the assumption of average weather conditions, we expect to cover almost the entire area covered by eboss [1-5] and shown in Figure 3.

The third and fourth years will be split evenly between observations of ELG plates and observations of the LRG and quasar plates. The scheduled time in which the SGC is visible will be dedicated to ELG targets while the NGC time will be dedicated to the LRG and quasar targets. At a rate of 300 plates per year, we expect the ELG program to be completed by the summer of 2018. Because the selection of ELG targets remains uncertain, we do not define the exact footprint of that survey at this time. For 2016-2018 LRG and quasar observations, we expect 300 plates to be completed in a $1500 \mathrm{deg}^{2}$ area of the NGC that lies just below the eboss 3 and eboss 5 regions. The exact area will depend on how much progress is made in the 2014-2015 and 2015-2016 observing seasons.

Observations of ELG targets will be completed before the summer of 2018, regardless of whether we meet the goal of 300 plates. By completing the ELG program in the fourth year, we establish a meaningful cosmological sample in the shortest possible time. The final two years will be dedicated entirely to the LRG and quasar targets, with the goal of covering as much SGC and NGC area as possible.

\section{SPECTROSCOPIC DATA QUALITY}

As explained in the appendix of Alam et al. (2015a), good fortune during 2010-2014 allowed the main BOSS program to finish early, leaving time for new dedicated spectroscopic observations. One hundred twenty eight plates were drilled for the SEQUELS program, covering the $466 \mathrm{deg}^{2}$ region bounded by eboss 4 and eboss 5 in Figure 3. In this section, we use the public SEQUELS data to present the quality of the LRG, clustering quasar, and Ly $\alpha$ forest quasar spectra expected in eBOSS. A mosaic of randomly selected spectra spanning the relevant range of redshift for each of these three samples is presented in Figure 5.

SEQUELS was designed as a pilot survey for eBOSS, using a slightly broader selection for LRG, clustering quasars, and Ly $\alpha$ forest quasars that was guaranteed to include the final eBOSS selection for these classes. Sixty six SEQUELS plates were completed before the end of SDSS-III and are included in DR12. Details for accessing these data are online ${ }^{79}$ and in Alam et al. (2015a). The remaining 62 plates will be observed at highest priority during the first year of eBOSS. The $466 \mathrm{deg}^{2}$ area will not be re-tiled with eBOSS-selected targets; instead, the SEQUELS targets will be integrated into future clustering analysis according to the same selection algorithms as those in eBOSS.

All SEQUELS targets are tracked by the dedicated EBOSS_TARGETO bitmask. In the 66 plates that were observed, fibers were placed on 11,687 unique targets from a modified version of the final eBOSS LRG selection algorithm. Although the LRG selection in SEQUELS is broader than in eBOSS, the eBOSS targets can be identified by objects with LRG_RIW (corresponding to bit number 2), extinctioncorrected magnitude $i_{\text {model }}<21.8$, and extinction-corrected magnitude $W 1<17.6$ (approximately 20.3 in AB). 10,873 of the SEQUELS LRG targets satisfy the final eBOSS LRG selection algorithm. Likewise, 19,461 unique clustering quasar targets, 6479 PTF-selected quasar targets, and 1368 reobserved

\footnotetext{
79 http://www.sdss.org/dr12/
}

Ly $\alpha$ quasars are found in SEQUELS. The clustering sample and the reobserved Ly $\alpha$ quasar sample are selected in an identical fashion to those in eBOSS, identified by QSO_EBOSS_CORE (bit 10) and QSO_REOBS (bit 12), respectively. The PTF sample contains a higher density of objects than in eBOSS and is difficult to reproduce without the variability parameters for each object. The variability parameters will be found in the final BOSS quasar catalog (Pâris et al. 2016, in preparation) when it is complete.

The SEQUELS spectra are the source of the redshift distributions for quasars presented in Table 1. Only SEQUELS data are presented in this section for the quasar samples. Both SEQUELS and proprietary eBOSS spectra were used to determine the redshift distributions for LRGs presented in Table 1, although the discussion of pipeline performance and the example spectra are based only on SEQUELS. The spectra of a large number of these objects were visually inspected to help characterize pipeline performance and to settle on the final redshifts of the samples. Pilot observations and visual inspection during the first few months of SDSS-IV led to the estimates of ELG redshift distributions presented in Table 2. Since the final ELG program is not decided, we postpone further discussion of those samples until future publications. Below, we describe the typical quality of LRG and quasar spectra and the interpretation of the automated redshifts and visual inspections that led to the $N(z)$ estimates assumed for eBOSS.

\subsection{LRG Spectra from SEQUELS}

The SEQUELS data are reduced by the same "idlspec2d" routines as those used in BOSS. The spectral templates described in Bolton et al. (2012) are fit to each reduced spectrum to derive a redshift and object classification. These templates were tuned for optimal performance in the BOSS galaxy sample and exceeded the predicted redshift success rates for even the most distant galaxies. The automated classification of the final BOSS CMASS galaxy sample shows $98.4 \%$ of objects with ZWARNING_NOQSO equal to zero, indicating that the automated redshift estimate is reliable when using a template suite that excludes quasar templates.

The choice of spectral templates was sufficient for BOSS, but is not optimized for the fainter, higher redshift LRG galaxies that comprise the eBOSS LRG sample. Of the 10,873 eBOSS LRG targets in SEQUELS, 7796 produced a ZWARNING_NOQSO value equal to zero in the automated reductions. A statistical error on the redshift estimate is provided by the automated classification routine for each of these galaxies. Tests in BOSS using repeat observations showed that these errors were underestimated by up to a factor of 1.34 for galaxies (Bolton et al. 2012). Assuming that the SEQUELS/eBOSS errors are underestimated by a factor of 1.34 , we find that the median redshift error is only $58 \mathrm{~km} \mathrm{~s}^{-1}$ and that only ten objects have a redshift error larger than $200 \mathrm{~km} \mathrm{~s}^{-1}$. The automated classification characterizes redshifts with a precision much better than required.

The single largest failure mode for the automated classifications is due to the inability to discriminate between best fit templates at different redshifts. Objects that fail according to this criteria are assigned a warning flag equal to two, which means the $\chi^{2}$ difference between the best spectral fit and the second best spectral fit is less than 0.01 per degree of freedom. This failure mode is not unexpected; the eBOSS LRG targets 


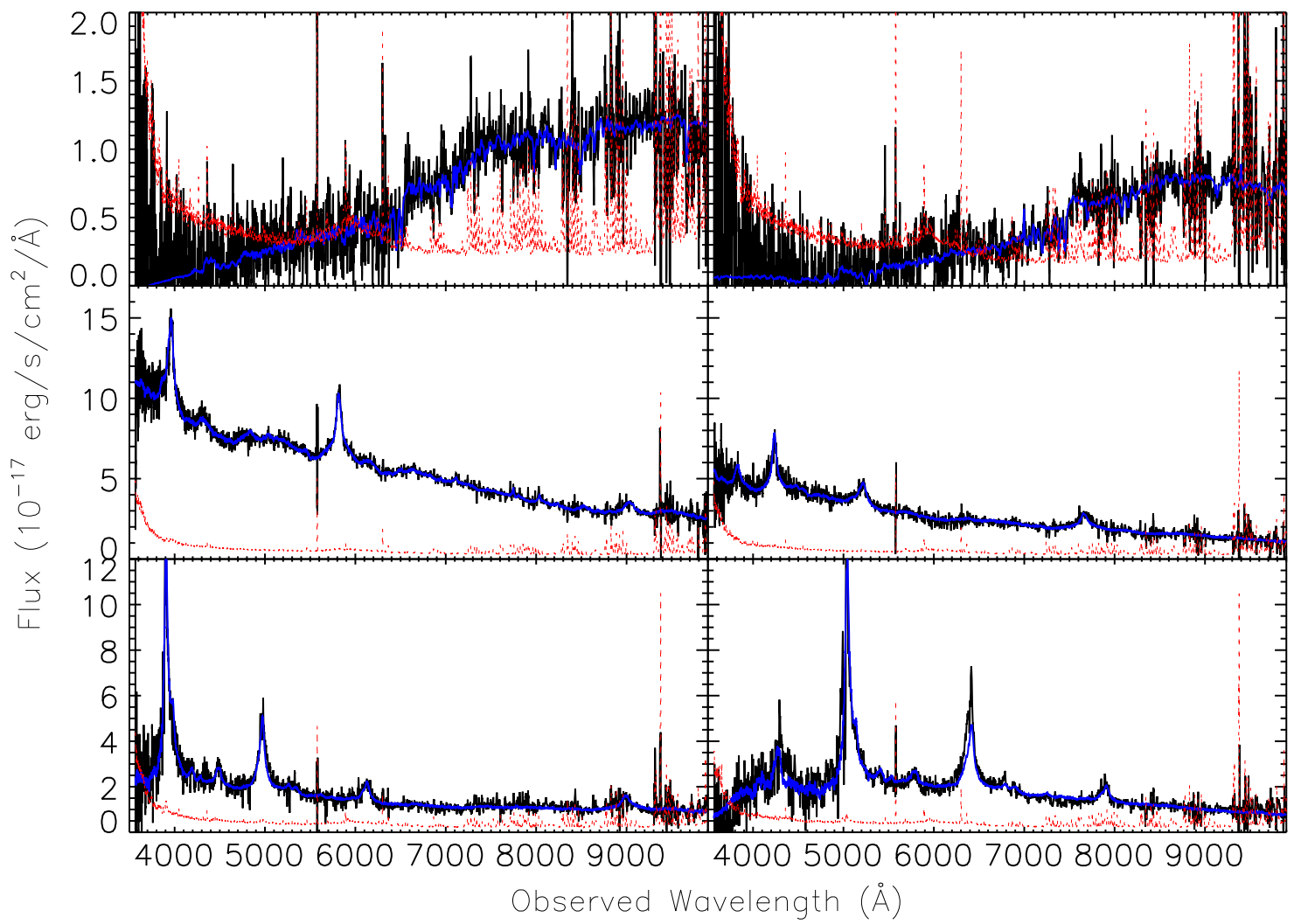

Figure 5. Examples of SEQUELS spectra that span the range of redshifts expected in the LRG, clustering quasar, and Ly $\alpha$ forest quasar samples. In each, the data are represented in black, the flux errors on each pixel in red, and the template in blue. A boxcar smoothing kernel of width 5 pixels has been applied to the data for illustrative effect. Each spectrum is classified with high confidence by the automated data reduction pipeline. Top left: an LRG at $z=0.64$. Top right: an LRG at $z=0.88$. Middle left: a quasar at $z=1.08$ identified by the QSO CORE selection algorithm. Middle right: a quasar at $z=1.74$ identified by the QSO CORE selection algorithm. Bottom left: a quasar at $z=2.21$ identified by the QSO_CORE selection algorithm. Bottom right: a quasar at $z=3.15$ identified by variability in the PTF imaging data.
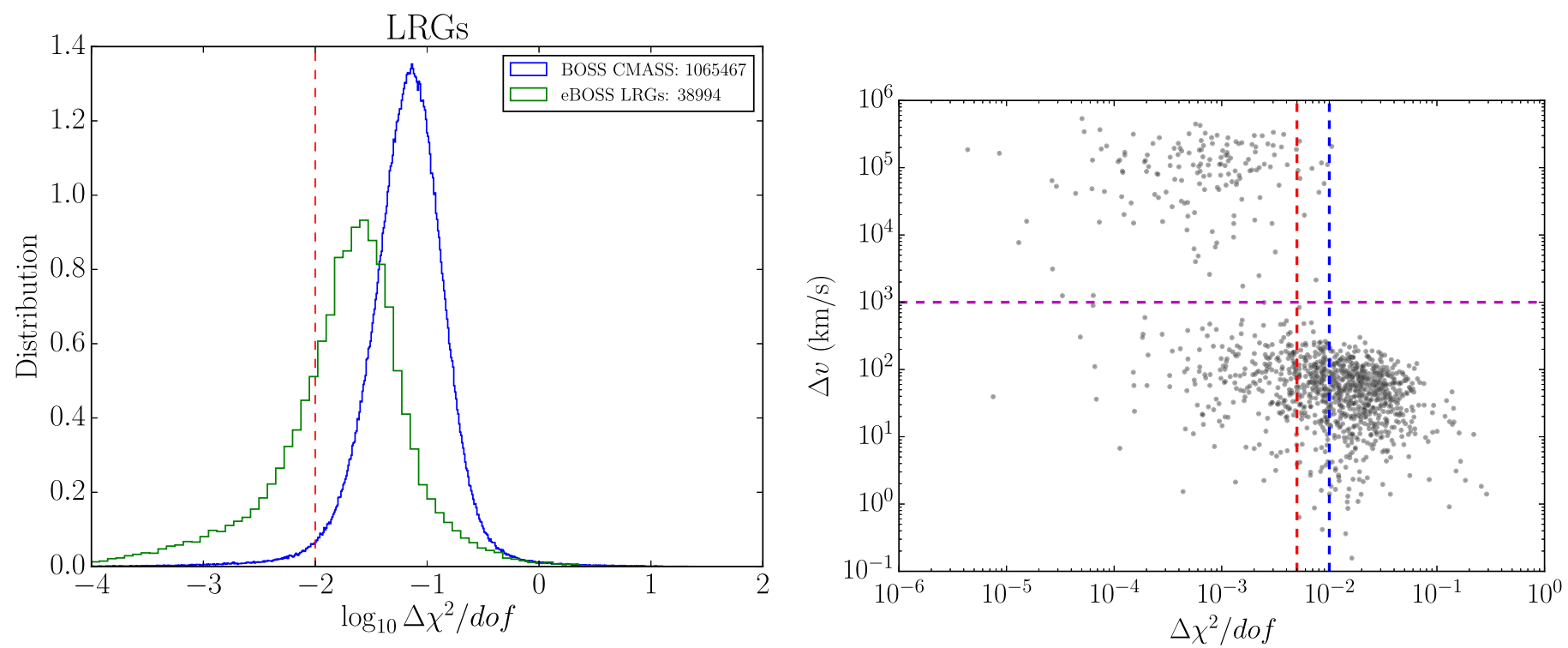

Figure 6. Left: the distribution of $\Delta \chi^{2}$ per degree of freedom for the BOSS sample of CMASS galaxies and the eBOSS LRG sample. The $\chi^{2}$ per degree of freedom value represents the difference between the best fit spectral template and the second best spectral template when quasar templates are excluded from the fits. Right: a scatter plot showing the difference in redshift $\left(\mathrm{km} \mathrm{s}^{-1}\right)$ between pairs of observations taken of the same LRG target. The two vertical lines represent threshold for the current $\Delta \chi^{2}$ ZWARNING_NOQSO (blue) and the more lenient threshold that increases the rate of redshifts that are considered reliable (red). The horizontal line (magenta) represents the limit at which redshift discrepancy is considered a catastrophic failure.

are intrinsically fainter than the BOSS galaxies and thus have a lower $\mathrm{S} / \mathrm{N}$ measurement of flux at each pixel in the spectrum. The faint spectra result in less pronounced absorption features that would otherwise provide a good redshift estimate and a reliance on broadband flux that is harder to fit as a function of redshift. The overall reduction of the $\chi^{2}$ difference with respect 
to the BOSS galaxy sample is shown in the left panel of Figure 6. The full distribution clearly shifts toward lower $\Delta \chi^{2}$, leading to a higher fraction of objects failing to meet the required difference of 0.01 per degree of freedom.

Of the 7796 objects that were given a ZWARNING_NOQSO value equal to zero, only 6096 fall in the redshift range $0.6<z<1.0$. The corresponding $56.1 \%$ combined targeting and spectroscopic efficiency would fall below our requirement (80\%) to obtain 300,000 spectroscopically confirmed LRG tracers. In particular, the fact that 1700 of the 10,873 spectra are given ZWARNING_NOQSO equal to zero and do not lie in the redshift range of interest makes it nearly impossible to meet the requirement for $80 \%$ completeness.

We further explored the quality of the automated classification in two ways to see how close the selection is to the required tracer density. First, we identified 591 LRG targets that were tiled on more than one plate and produced multiple spectra. We supplemented this sample by re-running the data reduction pipeline on four plates that were observed to more than twice the normal depth, dividing those exposures into unique coadds of the data. The data split produced 630 targets with multiple spectral classifications. We assessed the rate of catastrophic redshift failures by counting the fraction of objects for which a pair of observations produced discrepant redshifts. For the sample of 2442 total observations, we found 166 discrepant redshifts, corresponding to a catastrophic failure rate of $6.8 \%$ if we assume that consistent redshifts are correct. We further explored the rate of discrepant redshifts as a function of $\chi^{2}$ per degree of freedom. As shown in the right panel of Figure 6, the vast majority of discrepant redshifts occur for rchisq_NOQSO $<0.005$, implying that the threshold of 0.01 may be too strict. Considering that a spectrum has 4400 pixels over the range $3600<\lambda<10000 \mathrm{~A}$ before accounting for masked artifacts, reducing the threshold to 0.005 corresponds roughly to $\Delta \chi^{2}=20$. Filtering on targets with this new threshold, we find that the rate of ZWARNING_NOQSO failures drops from $28 \%$ to $16 \%$. Of the 1650 observations of objects where both repeated spectra were fit with rchisq_NOQSO $>0.005$, only ten produced discrepant redshifts, implying a catastrophic failure rate of $0.6 \%$, below the maximum allowed $(1 \%)$.

When reducing the threshold for rchisq_NOQSO, we reduce the rate of ZWARNING_NOQSO failures by nearly a factor of two and appear to meet the requirements for catastrophic redshift failures. However, even with this change we are still well below the required rate of spectroscopic confirmation. As a second test, we proceeded to visually inspect the LRG spectra from 18 different plates that obtained the deepest observations. The visual inspections were intended to provide insight into the spectral quality, the properties of the templates producing the best and second best fits, and to gauge the true redshifts of the LRG sample.

While it is impossible to run visual inspections in a reproducible and consistent fashion, we attempted to provide a scheme by which we could assess the range of likelihoods that a redshift is correct. We established a simple four-tiered redshift confidence metric $z_{\text {conf }}$.

1. $z_{\text {conf }}=0$ denotes a spectrum for which it is impossible to classify the type or determine a redshift. Of the LRG target spectra that were visually inspected, $6.6 \%$ were assigned $z_{\text {conf }}=0.88 \%$ of these spectra were given ZWARNING_NOQSO $>0$ in the automated classification.
2. $z_{\text {conf }}=1$ denotes a spectrum that is ambiguously classified, where more than one of the best-fit models is a possible fit. The redshift provided by the inspector is intended to be a best estimate of the correct redshift. $4.5 \%$ of the spectra were determined to be galaxies and assigned $z_{\text {conf }}=1.48 \%$ of these spectra were given ZWARNING_NOQSO $>0$ in the automated classification.

3. $z_{\text {conf }}=2$ denotes a spectrum where the redshift estimate is likely to be correct as there is no other template that provides an equally compelling fit. However, some degree of uncertainty remains because the best-fit spectral template does not reproduce all of the expected features. For example, the template may fit the continuum but certain absorption features may be ambiguous in the data. $12 \%$ of the spectra were determined to be galaxies and assigned $z_{\text {conf }}=2.23 \%$ of these spectra were given ZWARNING_NOQSO $>0$ in the automated classification.

4. Finally, $z_{\text {conf }}=3$ denotes a case where the redshift is estimated at very high confidence. $68 \%$ of the spectra were determined to be galaxies and assigned $z_{\text {conf }}=3$. $4.8 \%$ of these spectra were given ZWARNING_NOQSO $>0$ in the automated classification.

5. $8.9 \%$ of the objects were classified as stars. M-stars were the dominant stellar contaminant and were very easy to identify in visual inspection. $92 \%$ of the stars were given $z_{\text {conf }}=3$, but $42 \%$ were given ZWARNING_NOQSO $>0$. The high rate of ZWARNING_NOQSO was later found to be caused by a bug in the version of the pipeline used for DR12. This will be fixed for the next public data release.

Two redshift distributions are presented in Table 1. The more conservative estimate (higher rate of "Poor Spectra") assumes that any target given $z_{\text {conf }}>1$ is given the correct redshift. The less conservative estimate corresponds to all objects with $z_{\text {conf }}>0$. It is likely that the true distribution lies between these two bounds.

Visual inspection reveals at least three failure modes among the targets with small $\Delta \chi^{2}$. One failure mode occurs when the spectrum possesses a non-physical gradient in the flux at the bluest wavelengths or the reddest wavelengths. An example of one such failure is shown in Figure 7. One notes a clear identification of the $\mathrm{Ca} H \& \mathrm{~K}$ features around observed wavelength $7200 \AA$ in the left hand panel. However, the data deviate significantly from the template over the range 3600-4000 $\AA$, leading to an inflated $\chi^{2}$ estimate. The template in the right hand panel does not capture the narrow absorption features because the redshift is incorrect. Instead, the template is better fit to the excessive UV flux that is likely due to imperfect spectral extraction and not of astrophysical origins. In cases such as this, the polynomial term that accounts for uncertainties in flux calibration appears to be better coupled to incorrect galaxy templates than to a galaxy template at the correct redshift.

A second failure mode is shown in Figure 8. Because the sources in the LRG target sample are typically passive galaxies at a high redshift, the significance of the $4000 \AA$ break can be diminished due to low flux counts. In these cases, it becomes difficult to discriminate between the $4000 \AA$ break and G-band absorption extending to $4304 \AA$. A spectral template of a higher redshift galaxy with a suppressed G-band feature (right panel of Figure 8) can sometimes fit the observed spectrum nearly as well as a template with a stronger G-band feature and a correct fit to the $4000 \AA$ break. While the template that produces the 

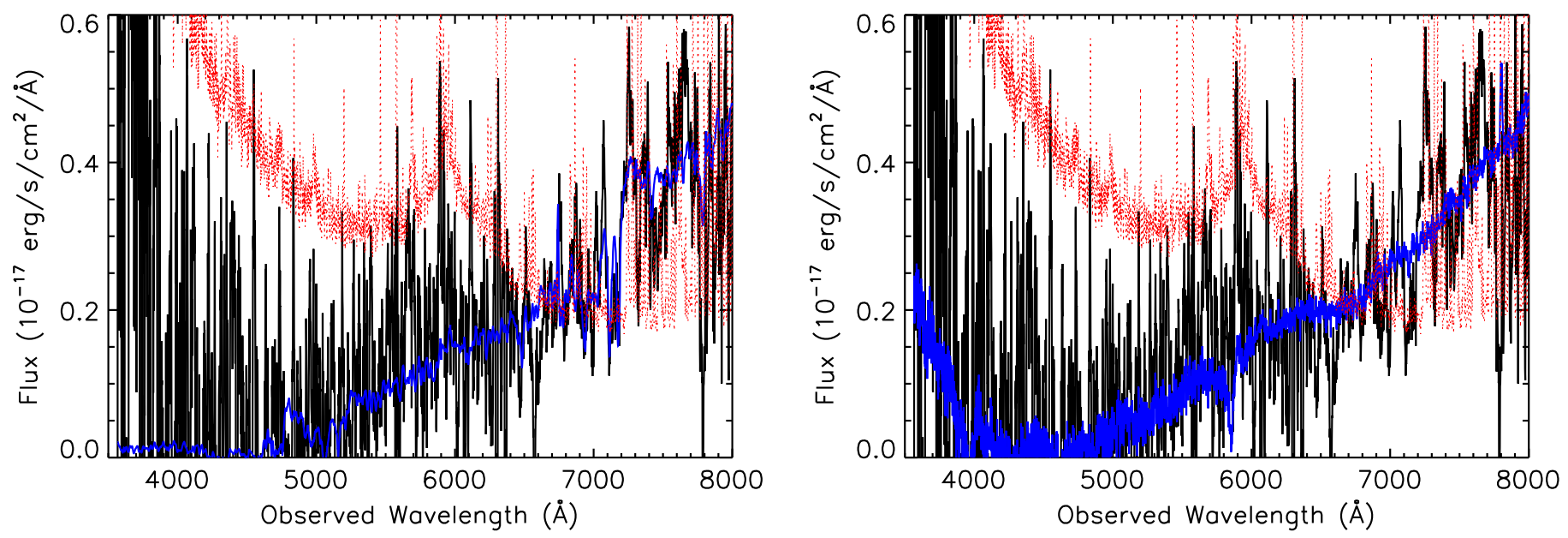

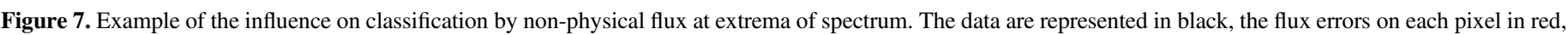

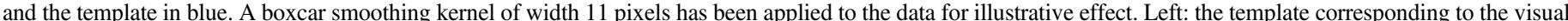

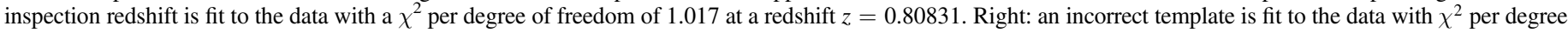
of freedom of 1.022 at $z=1.09220$, a difference from the first template small enough to trigger the ZWARNING_NOQSO flag.
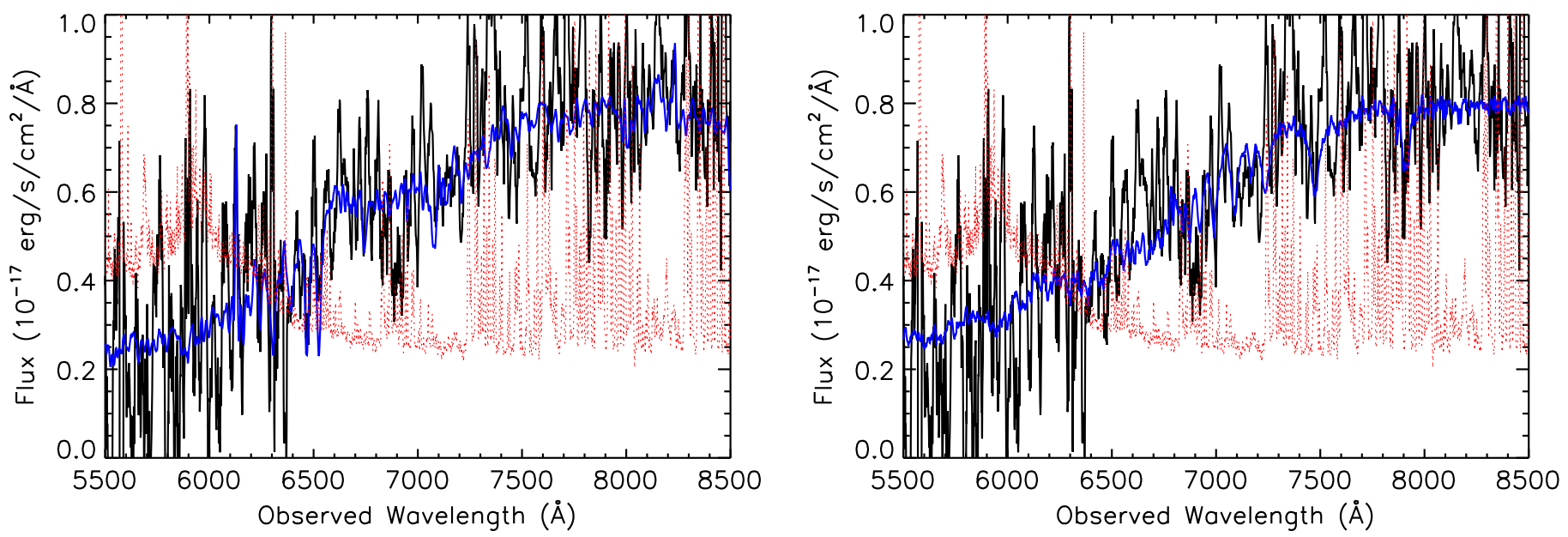

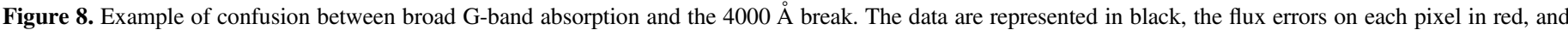

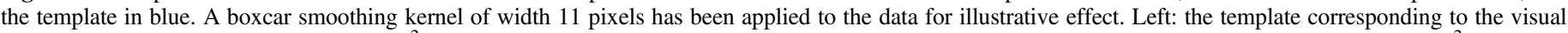

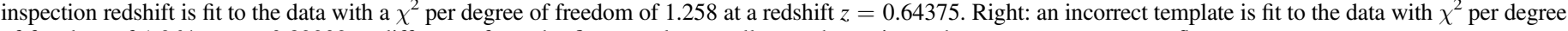
of freedom of 1.264 at $z=0.82202$, a difference from the first template small enough to trigger the ZWARNING_NOQSO flag.
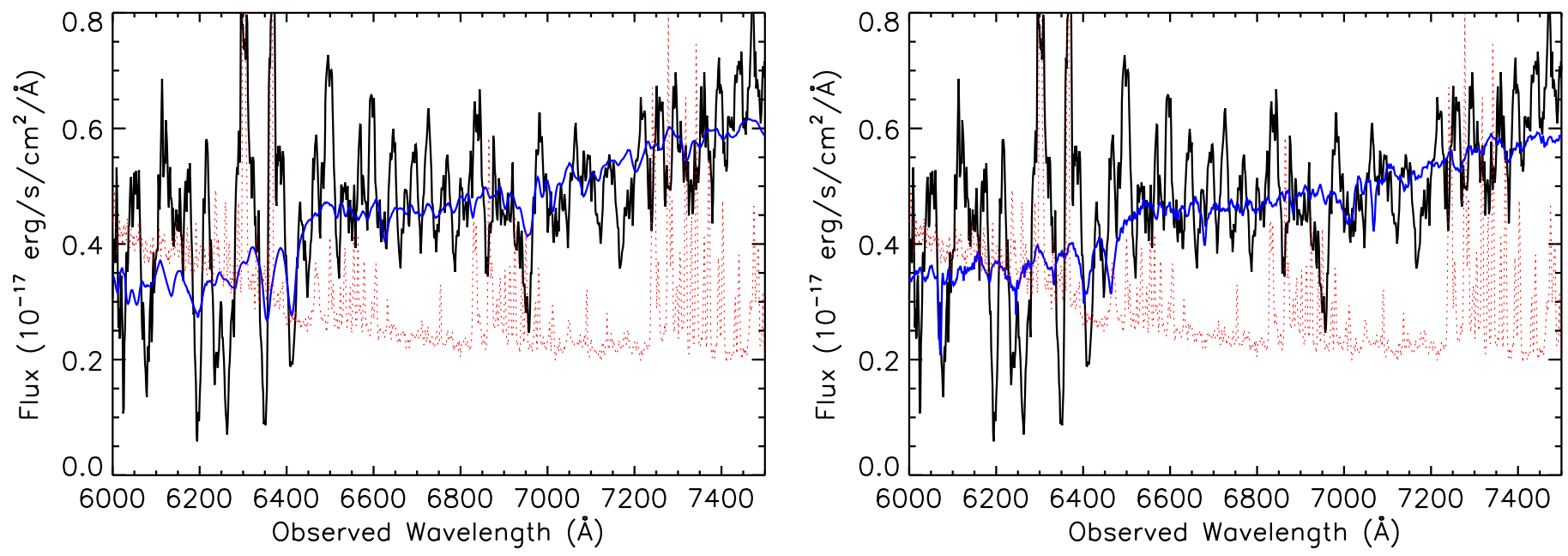

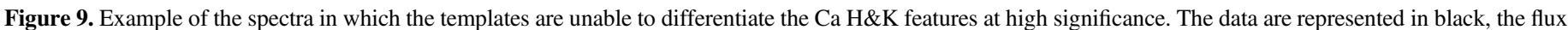

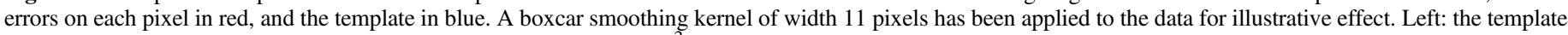

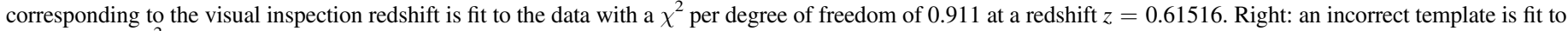
the data with $\chi^{2}$ per degree of freedom of 0.915 at $z=0.62806$, a difference from the first template small enough to trigger the ZWARNING_NOQSO flag. 
Table 5

Performance of the Automated Classification Scheme Applied to the SEQUELS Quasar Sample

\begin{tabular}{|c|c|c|c|c|}
\hline & \multicolumn{4}{|c|}{ Automatic Classification } \\
\hline & STAR & QSO & GALAXY & UNKNOWN \\
\hline \multicolumn{5}{|c|}{ Visual inspection result } \\
\hline$? / \mathrm{BAD}$ & $78(0.2 \%)$ & $102(0.3 \%)$ & $427(1.3 \%)$ & $486(19.9 \%)$ \\
\hline STAR & $9845(29.2 \%)$ & $19(0.05 \%)$ & $159(0.5 \%)$ & $189(7.7 \%)$ \\
\hline QSO & $37(0.1 \%)$ & $18,475(54.7 \%)$ & $103(0.3 \%)$ & $1718(70.3 \%)$ \\
\hline GALAXY & $13(0.04 \%)$ & $24(0.07 \%)$ & $4390(13.0 \%)$ & $50(2.1 \%)$ \\
\hline
\end{tabular}

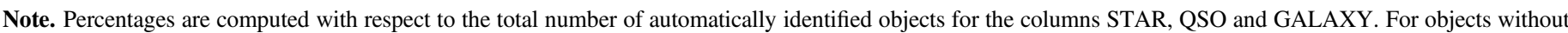

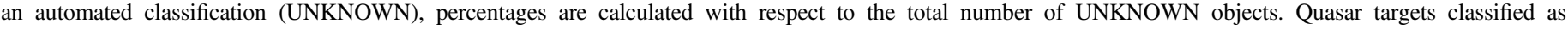
UNKNOWN by the automated classification are visually inspected.

stronger G-band absorption profile is more physically motivated than the higher redshift template, the current reduction pipeline has no mechanism in place to impose a prior in favor of the correct redshift.

Finally, the third failure mode occurs because the majority of the signal is found in the broadband flux as opposed to the monochromatic $\mathrm{Ca} \mathrm{H} \& \mathrm{~K}$ features. In cases such as the spectrum shown in Figure 9, there is little power to statistically discriminate between a fit that correctly places both $\mathrm{Ca} \mathrm{H} \& \mathrm{~K}$ features and a fit that staggers the template with an incorrect fit to a single feature. In many cases, the G-band absorption line

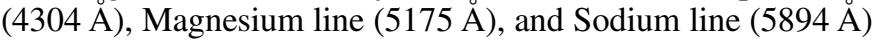
provide additional constraints on the redshift, but these lines become ambiguous at higher redshifts where sky subtraction residuals are more significant.

As will be discussed in Section 6.2, we are now improving the algorithms for spectral extraction and classification to mitigate these classes of failure modes. We are confident that we can increase the rate of reliable classifications beyond what is presented in DR12 and are optimistic that we can achieve statistics comparable to the $z_{\text {conf }}>0$ redshift identification rate from the visual inspections.

\subsection{Quasar Spectra from SEQUELS}

Quasar targets in BOSS were observed at a rate of roughly 200 per plate, totaling nearly 500,000 over the full program. Each of these spectra was visually inspected and given a classification (quasar, star, or galaxy) and a redshift estimate with a documented degree of confidence (Pâris et al. 2012, 2014). We also relied on visual inspection to flag quasar sightlines with peculiarities such as damped lyman-alpha and BAL systems that could not be classified with the automated redshift classification (Bolton et al. 2012). The photometric and spectroscopic parameters of each object were recorded for use by all members of the collaboration and released in public form on a regular basis. The process was undertaken primarily by two members of the collaboration and proved to be very timeconsuming.

The quasar redshift distributions presented in Section 4.2 were derived from visual inspections of all SEQUELS plates observed during BOSS. Even the faintest quasars $\left(r_{\mathrm{PSF}}=22\right)$ can be confidently classified in visual inspections; $95.7 \%$ of the full quasar sample (including TDSS, SPIDERS, and PTFselected objects) can be identified with high confidence. A comparison of the automated pipeline to the results of visual inspection reveal a high level of agreement. The redshift estimates from the spectroscopic pipeline are consistent with the visual inspection estimates to within $1000 \mathrm{~km} \mathrm{~s}^{-1}$ for
98.7\% of QSO_CORE quasars $\left(r_{\mathrm{PSF}}<22\right)$. At this early stage, the spectroscopic pipeline nearly meets the requirement presented in Section 3.2 that $<1 \%$ of objects are given catastrophically incorrect redshifts.

The density in eBOSS and SEQUELS increases to roughly 600 quasar targets per plate which motivated the collaboration to revisit the automated classification schemes and significantly decrease the level of visual inspection. To fully transition to this new scheme, we evaluated the pipeline performance against visual inspections to determine the source of catastrophic failures and systematic uncertainties in redshift estimates. We found patterns in catastrophic failures associated with the class of object reported by the pipeline, the redshift estimate, and the second, third, fourth and fifth best estimates. By applying the following criteria, we are able to improve the consistency between the pipeline classifications and the visual inspections.

1. Objects identified as a star in the best estimate are assumed to be a star.

2. Objects identified as a galaxy with $z<1$ in the best estimate are assumed to be a galaxy at $z<1$.

3. Objects identified as a galaxy with $z>1$ in the best estimate are assumed to be a galaxy if one of the next four classifications is also a galaxy.

4. Objects identified as a quasar with $\mathrm{ZWARNING}=0$ are assumed to be a quasar unless two or more alternate identifications are given a class of star. In these cases, the object is assumed to be a star.

5. Objects identified as a quasar with ZWARNING $>0$ are assumed to be a star if two or more alternate identifications are given a class of star.

6. Objects that fail to meet these criteria are followed up with visual inspection.

The results of this classification scheme applied to SEQUELS data is presented in Table 5. This new classification provides identifications for $93 \%$ of all the quasar targets. Using the visual inspections as a baseline, among those automatically classified targets, $96.9 \%$ are correctly characterized by our new algorithm. It is specifically designed to pick up actual quasars and to limit the fraction of contaminants in the quasar sample and lost quasars: $54.7 \%$ are actual quasars that are confirmed after visual inspection. A total of $0.42 \%$ quasar targets are wrongly classified as quasars by our automated scheme. Those contaminants are largely dominated by spectra with very low signal-to-noise ratios, and could also not be identified after visual inspection. Our procedure misidentifies $0.40 \%$ of quasar targets that are confirmed to be quasars after visual inspection. 
Among the $7 \%$ of all quasar targets that still require visual inspection, $70.3 \%$ are true quasars. Assuming that the identification error from the visual inspection is negligible, the total fraction of contamination of the quasar sample and loss is below the $1 \%$ of the overall sample as required in Section 3.2.

So we can expect that eBOSS quasar redshifts will be robust, but an additional requirement noted in Section 3.2 is that they should also have a precision of order $300-600 \mathrm{~km} \mathrm{~s}^{-1}$. Based on repeat spectroscopy, the rms scatter of BOSS pipeline redshift estimates for quasars is a factor of 2 higher than the reported statistical errors (Bolton et al. 2012). Taking into account these underestimated errors, the typical statistical precision is between $\sigma_{v} \sim 100$ and $\sigma_{v} \sim 300 \mathrm{~km} \mathrm{~s}^{-1}$ in the redshift range $1<z<2$, depending on the luminosity of the source. If truly representative of the redshift errors, this level of precision would be sufficient to avoid degradation in the clustering signal.

The reported statistical precision does not necessarily reflect the true accuracy on a quasar redshift due to possible systematic uncertainties in the redshifting templates (e.g., Hewett \& Wild 2010). Highly ionized gas in the broad-line region of quasars is subject to radiation-driven winds (e.g., Proga 2003). It is therefore likely that the measured redshifts largely determined by these emission lines are offset from the systemic redshift. Such an effect has been reported in the redshift estimates of $z>2.1$ quasars used in Ly $\alpha$ forest-quasar cross correlations (Font-Ribera et al. 2013). Because Mg II is ionized at lower energies and is largely present at larger separations from the central black hole, $\mathrm{Mg}$ II emission lines are likely the least affected broad emission lines by systematic offsets. Various studies have estimated that the $\mathrm{Mg}$ II emission line is offset by an amount varying from -100 to $+50 \mathrm{~km} \mathrm{~s}^{-1}$ with respect to the quasar systemic redshift (e.g., McIntosh et al. 1999; Hewett \& Wild 2010; Font-Ribera et al. 2013). The upper bound on the statistical errors of quasar redshift estimates can be therefore estimated directly from fits to the $\mathrm{Mg}$ II emission lines.

In the redshift range of interest for eBOSS quasar clustering, the $\mathrm{Mg}$ II emission line is always covered in the spectra. Hence, it can be directly used to estimate quasar redshifts. In order to estimate the statistical error on $\mathrm{Mg}$ II-based redshifts, we use the spectra of 472 quasars in the redshift range $0.9<z<2.2$ taken as part of the SDSS Reverberation Mapping Project (Shen et al. 2015). Thirty spectroscopic epochs were taken in SDSS-III for each of those quasars with a homogeneous $\mathrm{S} / \mathrm{N}$ distribution corresponding to twice the normal BOSS and eBOSS exposure depth. The redshift and $g$-band magnitude distributions are comparable with the eBOSS sample. By fitting the $\mathrm{Mg}$ II line with a linear combination of five principal components, its redshift is measured at the position of the maximum flux of the emission line as described in Pâris et al. (2012). We estimate the rms scatter of the Mg II-based redshifts as a function of $g$-band magnitude and quasar redshift in Figure 10 (red diamonds). The redshift errors increase with $g$ band magnitude and vary from $\sim 80$ to $\sim 300 \mathrm{~km} \mathrm{~s}^{-1}$. This behavior is expected since the $\mathrm{S} / \mathrm{N}$ decreases for fainter objects. Redshift errors also increase from $\sim 130 \mathrm{~km} \mathrm{~s}^{-1}$ at $\mathrm{z}=1$ to $\sim 270 \mathrm{~km} \mathrm{~s}^{-1}$ at $\mathrm{z}=2.2$. Larger redshift errors for quasars at $z \geqslant 2$ are measured because the $\mathrm{Mg}$ II line lies in the red part of eBOSS spectra where the sky subtraction is noisy and/or imperfect and makes the line fitting more difficult.
Redshift errors are also larger at $z \sim 1.2$ when the $\mathrm{Mg}$ II emission line moves from the blue arm to the red arm of the eBOSS spectrograph.

The statistical errors on redshift measurements based on $\mathrm{Mg}$ II emission line fits are well within the requirements described in Section 3.2. However, this emission line is not always detected, especially in the spectrum of faint quasars at $z \geqslant 2$ when the $\mathrm{Mg}$ II line is at its red end. We thus consider redshifts estimated with quasar templates in order to overcome this issue. These templates have been calibrated with the $\mathrm{Mg}$ II emission line (see details in Pâris et al. 2012). We estimate the statistical error on those redshift measurements in the same manner as for the $\mathrm{Mg}$ II-based estimates. As expected, templatebased redshifts are more stable (black squares in Figure 10). The rms scatter increases from $\sim 60$ to $\sim 100 \mathrm{~km} \mathrm{~s}^{-1}$ for $18<g<22$ (left panel). There is no obvious redshift dependence of the statistical error with an average of $90 \mathrm{~km} \mathrm{~s}^{-1}$ (right panel). Despite this apparent better behavior of statistical errors for template-based redshifts, one significant drawback comes from astrophysical effects. Intrinsic emission line shifts depend on quasar luminosity (e.g., Hewett \& Wild 2010). Offsets can be accurately reproduced with templates if the whole luminosity range of interest is covered by the training sample, which is not the case of our current templates. The most affected emission line is $\mathrm{C} \operatorname{IV} \lambda 1550$ that is blueshifted by several hundreds of $\mathrm{km} \mathrm{s}^{-1}$ with respect to the systemic redshift (e.g., Richards et al. 2011). When this line enters in the spectrum, it may affect the accuracy of template-based redshift measurements and thus, it may cause an additional source of systematics that is redshift dependent. In order to quantify this effect, we measure $\mathrm{Mg}$ II- $^{-}$and template-based redshifts of quasars in the SEQUELS sample. We then measure the redshift evolution of the scatter between $\mathrm{Mg}$ II-based and templatebased redshifts (Figure 11). Between $z=0.9$ and $z=1.5$, redshift errors are below $300 \mathrm{~km} \mathrm{~s}^{-1}$. At larger redshift, the $\mathrm{C}$ IV line is covered by eBOSS spectra and redshift errors increase up to $600 \mathrm{~km} \mathrm{~s}^{-1}$. This demonstrates that template-based redshifts are dominated by astrophysical effects. Templates need to be improved to reduce these systematics. Nevertheless, redshift requirements (and high level BAO projections) noted in Section 3.2 are set to our current redshift accuracy (red line in Figure 11).

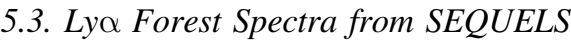

The Ly $\alpha$ forest sample differs from the LRG, ELG, and clustering quasar samples in that it will be used to increase the density of a BOSS sample that is already being used to measure the BAO distance scale. The analysis tools and spectral classification are well advanced for this sample of targets and are described in recent results. For example, three techniques for determining the underlying quasar continuum in the Ly $\alpha$ forest region were compared in the latest BAO measurement (Delubac et al. 2015a). Blomqvist et al. (2015) explore a model in Fourier space to correct broadband distortion introduced by continuum fitting. Implementing the technique on simulated spectra, they find that the linear bias parameter $b_{F}$ and the RSD parameter $\beta_{F}$ can be recovered with systematic errors less than $0.5 \%$. Astrophysical effects (quasar continuum diversity and high-density absorbers) and instrumental effects (noise, spectral resolution, and data reduction artifacts) that affect estimates of the Ly $\alpha$ forest auto-correlation function were quantified through catalogs of mock spectra (Bautista et al. 2015). 

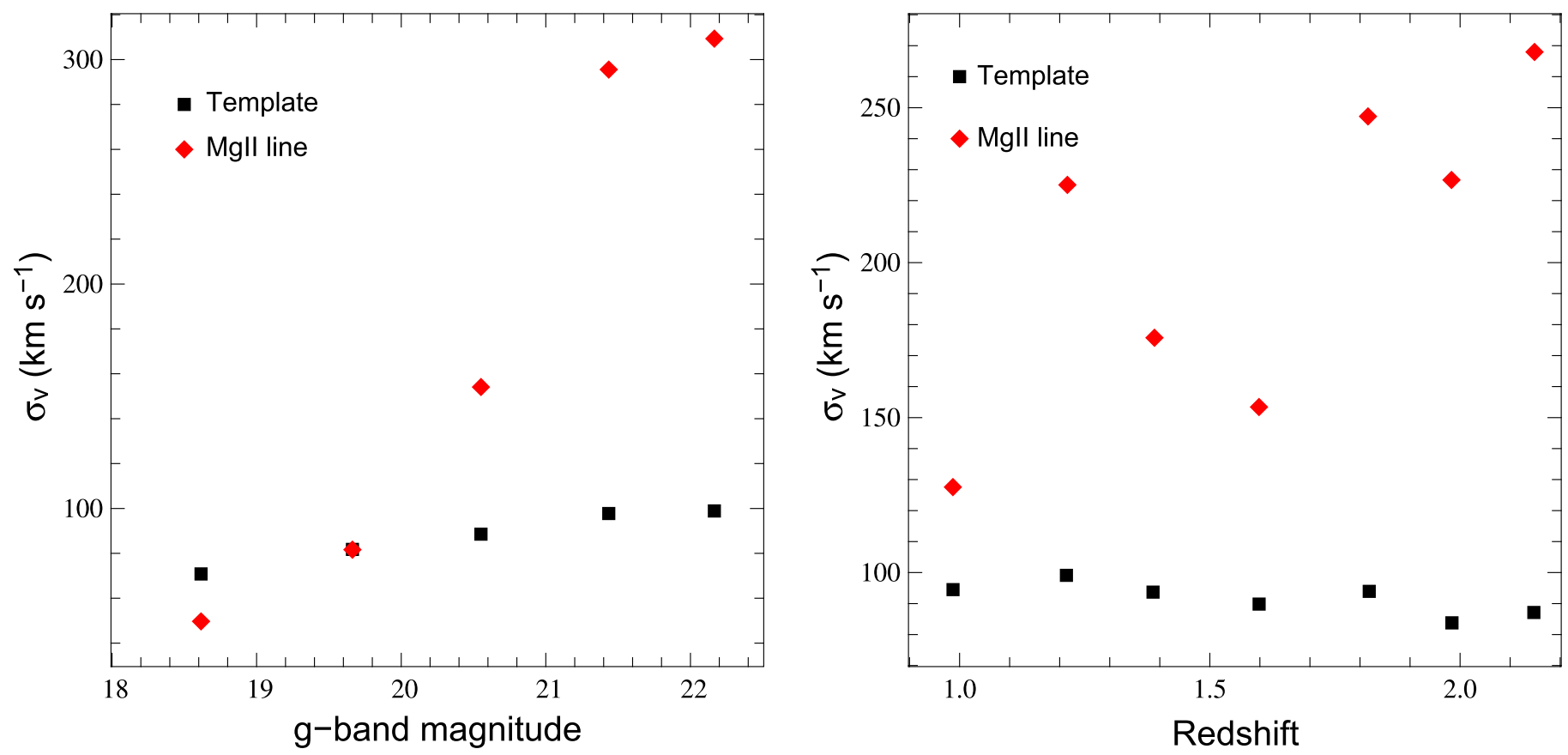

Figure 10. The statistical error on redshift estimates for the quasar sample for template-based (black squares) and Mg II-based (red diamonds) redshift estimates. Left: The redshift error as a function of $g$-band magnitude. Right: The redshift error as a function of redshift.

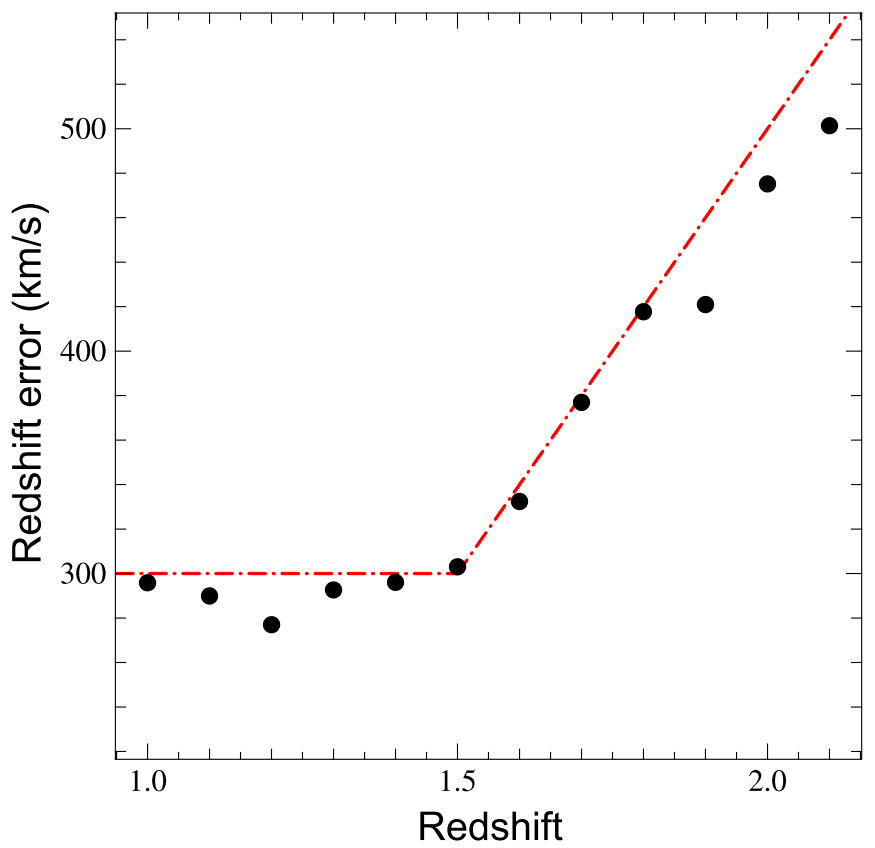

Figure 11. Redshift evolution of the statistical error on systemic redshift estimates for the quasar sample based on the comparison of template-based and $\mathrm{Mg}$ II-based redshit estimates. Black points are the statistical errors derived from the data. The red line shows the redshift evolution used for the cosmological forecasts described in Section 3.2.

Analysis in SDSS-IV will be performed jointly on the BOSS and eBOSS Ly $\alpha$ forest samples; modeling of the astrophysical and instrumental contributions to the measured clustering signal will continue to evolve.

The sample of $z>2.1$ quasars also provided the first opportunity in SDSS to measure BAO through cross- correlation of different tracers. The target sample produces both direct tracers of the underlying density field (quasars themselves) and illumination of neutral hydrogen through the Ly $\alpha$ forest. The large-scale cross-correlation of quasars with the $\operatorname{Ly} \alpha$ forest absorption was first measured to comoving separations of $80 h^{-1} \mathrm{Mpc}$ (Font-Ribera et al. 2013). The technique was later scaled to larger separations with a larger sample of quasars to measure $\mathrm{BAO}$ and provide constraints on $H(z)$ and $d_{A}(z)$ as discussed in Section 2.2.

The measurement of $\mathrm{BAO}$ with $\mathrm{Ly} \alpha$ forest in autocorrelation and in cross-correlation with quasars are both shot-noise limited and produce nearly independent constraints on the distance scale. The final BOSS DR12 sample is expected to produce combined constraints on $H(z)$ and $d_{A}(z)$ with a precision of $2.0 \%$ and $2.5 \%$, respectively. One can project the relative improvement offered by the new eBOSS $z>2.1$ quasar sample by evaluating the number density and depth of spectra compared to those in BOSS. The observed number density of quasars can be computed from the redshift distributions found in Table 1 and the tiling efficiencies in Table 3. The density of quasars from each sample after accounting for tiling efficiency is shown in Table 6. SEQUELS observations are used to assess the depth of spectra.

We follow the formalism presented in McQuinn \& White (2011) to estimate the fractional improvement on the BOSS $\mathrm{BAO}$ distance precision from the auto-correlation when adding eBOSS Ly $\alpha$ forest spectra. The BAO uncertainties are forecast based on the number of modes available given the survey volume and the signal-to-noise per mode $(\mathrm{S} / \mathrm{N})$. In the case of $\mathrm{Ly} \alpha$ forest spectra, the $\mathrm{S} / \mathrm{N}$ scales as

$$
(\mathrm{S} / \mathrm{N})^{2}=\frac{P_{F}^{2}(k)}{2 P_{\text {tot }}^{2}(k)}
$$


Table 6

Densities of Quasars and Lines of Sight to Ly $\alpha$ Forest in BOSS and the Various EBOSS Samples

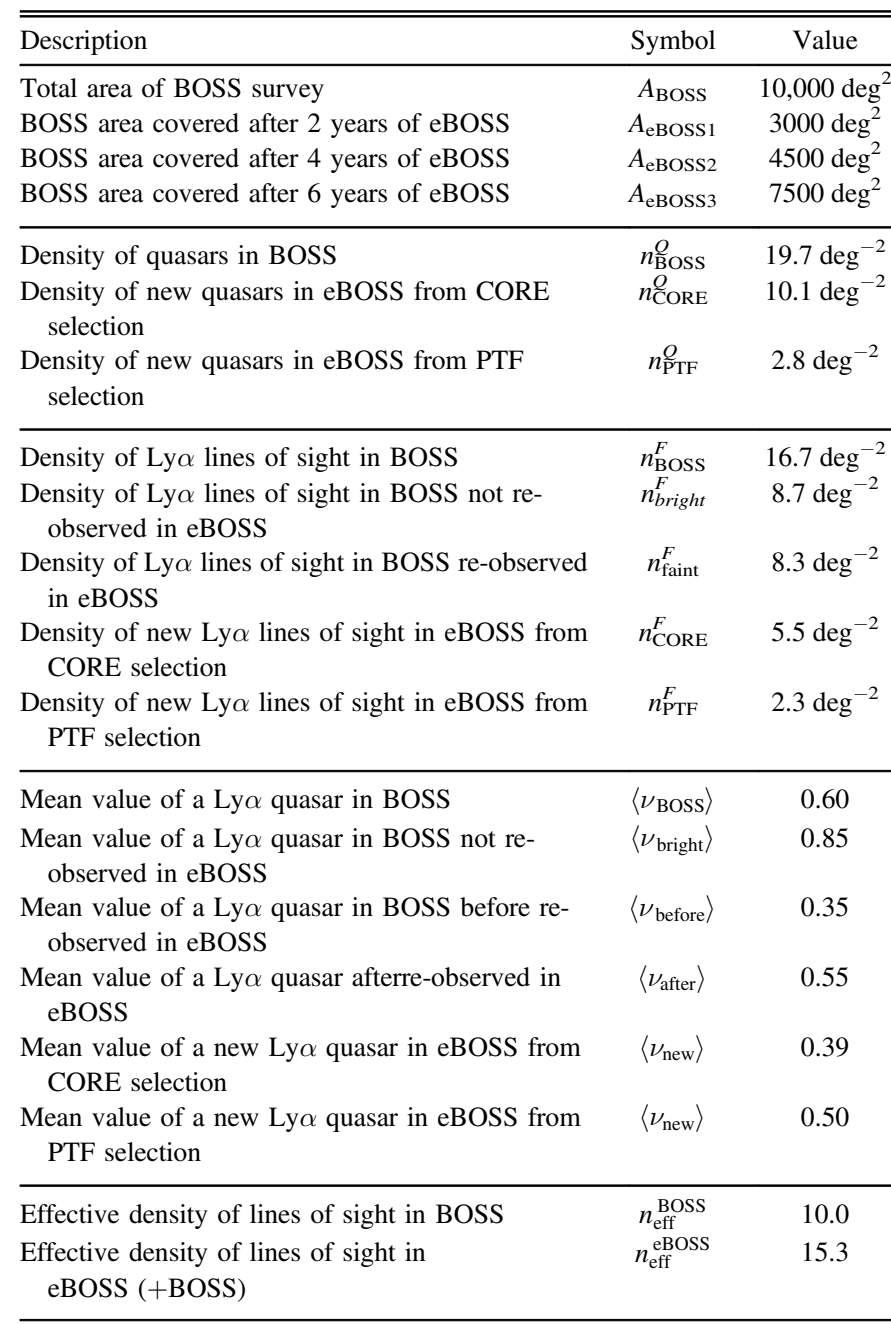

Note. The density of quasars $\left(n_{Q}\right)$ includes BAL quasars at redshifts $z>2.0$. The density of lines of sight $\left(n_{F}\right)$ include only quasars with $z>2.1$ that are not BAL quasars. BOSS numbers include the true non-BAL population while the eBOSS numbers include a $13 \%$ reduction in the observed number density to account for the predicted rate of BAL contamination.

where $P_{F}(k)$ is the flux power spectrum. $P_{\text {tot }}(k)$ is the variance of a given mode approximated by the combination

$$
P_{\text {tot }}(k)=P_{F}(k)+\frac{P_{1 \mathrm{D}}\left(k_{\|}\right)}{n_{\mathrm{eff}}},
$$

where $P_{1 \mathrm{D}}\left(k_{\|}\right)=P_{1 \mathrm{D}}$ is the $1 \mathrm{D}$ power along the line of sight. The effective angular density of lines of sight, $n_{\mathrm{eff}}$, is defined

$$
n_{\mathrm{eff}}=n^{F}\langle\nu\rangle=n\left\langle P_{1 \mathrm{D}} /\left(P_{1 \mathrm{D}}+P_{N}^{F}\right)\right\rangle .
$$

The average surface density of quasars used to measure absorption in the Ly $\alpha$ forest is represented by $n^{F}$ and $P_{N}$ is a weighted average of the noise power $\left(P_{N}=d x /(\mathrm{SNR})^{2}\right)$. The mean pixel width within the forest is recorded as $d x$ (units of $\mathrm{Mpc} h^{-1}$ ) and SNR is the mean signal-to-noise ratio per pixel within the forest. In a survey that is dominated by shot noise, the $\mathrm{S} / \mathrm{N}$ per Fourier mode should be roughly proportional to $n_{\text {eff }}$ at a given redshift. Although the calculations are redshift dependent, we assume that the redshift distributions in eBOSS are similar to BOSS so the uncertainties of eBOSS relative to BOSS should scale as the inverse ratio of $n_{\text {eff }}$ in the areas of overlap.

In studying the precision of $\mathrm{BAO}$ measurements in the $\mathrm{Ly} \alpha$ auto-correlation with the addition of eBOSS, we simply determine $n_{\text {eff }}^{\mathrm{eBOSS}}$ in the area $\left(A_{\mathrm{eBOSS}}\right)$ covered by BOSS and eBOSS and $n_{\text {eff }}^{\text {BOSS }}$ in the area ( $\left.A_{\text {BOSS }}\right)$ covered uniquely by BOSS. Since the two regions will be independent, we can compute the uncertainty on the BAO in eBOSS relative to BOSS according to

$$
\begin{aligned}
& \frac{\sigma_{\mathrm{eBOSS}}^{2}}{\sigma_{\mathrm{BOSS}}^{2}} \\
& =\frac{A_{\mathrm{BOSS}}\left(n_{\mathrm{eff}}^{\mathrm{BOSS}}\right)^{2}}{A_{\mathrm{eBOSS}}\left(n_{\mathrm{eff}}^{\mathrm{eBOSS}}\right)^{2}+\left(A_{\mathrm{BOSS}}-A_{\mathrm{eBOSS}}\right)\left(n_{\mathrm{eff}}^{\mathrm{BOSS}}\right)^{2}} \\
& =\frac{1}{f_{A} f_{n}^{2}+\left(1-f_{A}\right)},
\end{aligned}
$$

where we have defined $f_{A}=A_{\text {eBoss }} / A_{\text {BOss }}$ and $f_{n}=n_{\text {eff }}^{\text {eBOSS }} / n_{\text {eff }}^{\text {BOSS }}$.

The depth of these SEQUELS measurements for each target sample was used to compute the average effective value of each Ly $\alpha$ forest line of sight according to Equation (4). Using the observed densities $n^{F}$ found in Table 6, we find $n_{\text {eff }}^{\text {BOSS }}=10.0$, $n_{\mathrm{eff}}^{\mathrm{eBOSS}}=15.3$, and $f_{n}=1.53$. The area covered in eBOSS will progress according to the survey strategy presented in Section 4.5. $f_{A}$ will be equal to $0.3,0.45$, and 0.75 after the first, second, and third public data releases, respectively.

In the case of the cross-correlation, the $\mathrm{S} / \mathrm{N}$ per Fourier mode should be roughly proportional to the sampling of the density field. Since both the number density of quasars and the measurements of the Ly $\alpha$ forest contribute, the term $n_{\mathrm{eff}}$ from the auto-correlation gets replaced by what is effectively the geometric mean between the effective number density of $\operatorname{Ly} \alpha$ forest lines of sight and the quasar density. The last form of Equation (5) can be simply rewritten as

$$
\frac{\sigma_{\mathrm{eBOSS}}^{2}}{\sigma_{\mathrm{BOSS}}^{2}}=\frac{1}{f_{A} f_{n} f_{q}+\left(1-f_{A}\right)}
$$

where we have defined the additional parameter as the density of quasars in eBOSS relative to BOSS, $f_{q}=n_{\mathrm{eBOSS}}^{Q} / n_{\mathrm{BOSS}}^{Q}$. The values of $f_{A}$ for each incremental data release are of course the same as in the auto-correlation. Using the number density of quasars in the second block of Table 6, we find $n_{\text {BOSS }}^{Q}=19.7 \mathrm{deg}^{-2}, n_{\mathrm{eBOSS}}^{Q}=32.6 \mathrm{deg}^{-2}$, and $f_{q}=1.65$. The additional quasars from eBOSS will therefore have a slightly higher impact on the cross-correlation measurement than the auto-correlation measurement.

For the auto-correlation, we expect the error on the BAO distance scale from BOSS to be reduced by factors 1.18, 1.27, and 1.42 for the two year, four year, and six year increments in the program. For the cross-correlation, we expect the error to be reduced by factors $1.21,1.30$, and 1.46 . Because the improvements in the auto- and cross-correlation are so similar, we average the two and assume improvements of 1.19, 1.28, and 1.44 on the distance scale determined from the joint analysis. These values are used to project the BAO distance precision from Ly $\alpha$ forest data in Section 7. 
Table 7

Comparison of Expected EBOSS Performance Compared to Requirements for High-level BAO Measurements

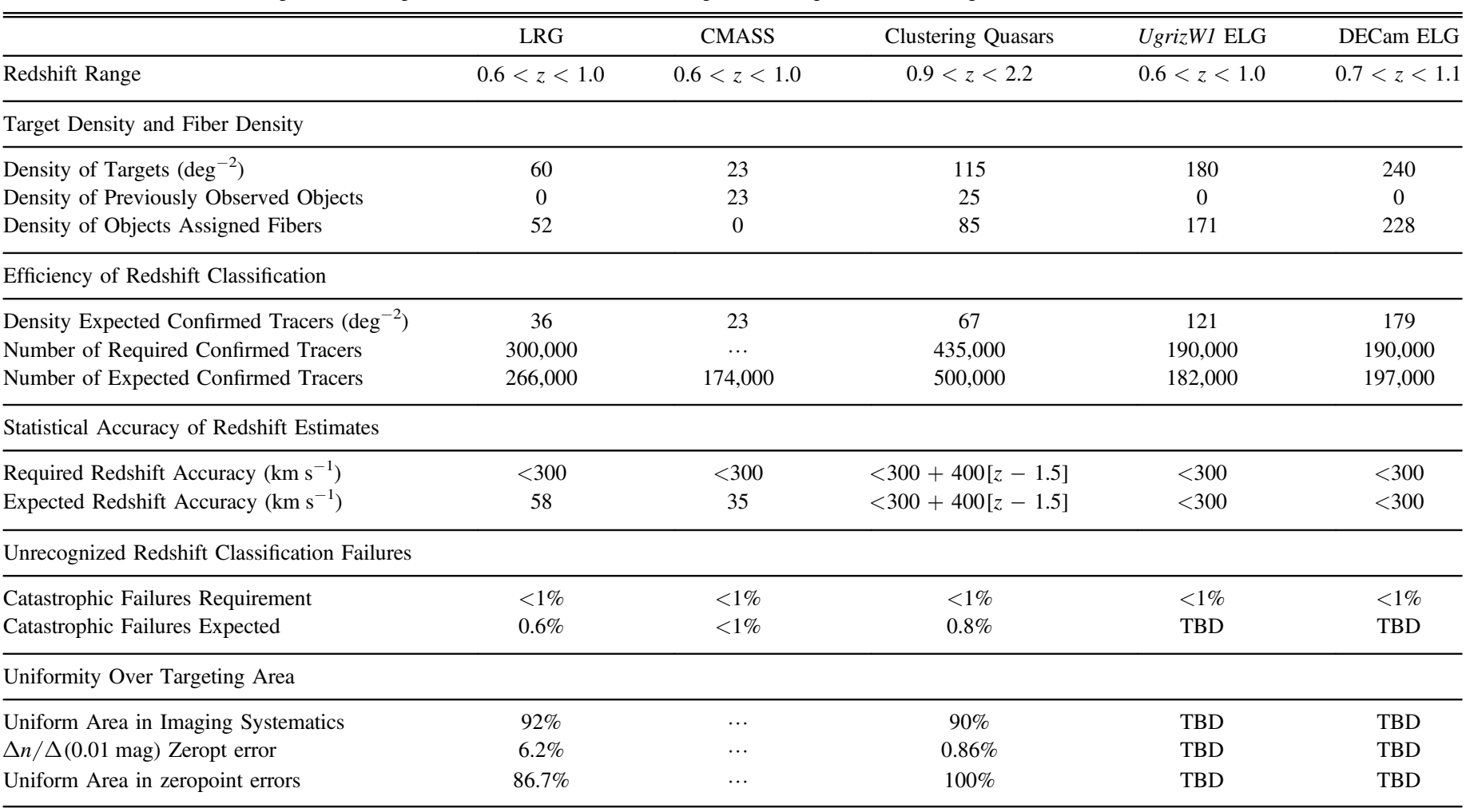

\section{SURVEY METRICS AND FUTURE DEVELOPMENT EFFORTS}

\subsection{Data Quality Compared to Requirements}

The high-level goals of eBOSS are to obtain BAO distance measurements with $1 \%$ precision using an LRG sample, with $2 \%$ precision using an ELG sample, and with $2 \%$ precision using a quasar sample. A number of low-level metrics must be satisfied to meet these goals. The redshift range and number of spectroscopically confirmed objects required for each class of tracer was presented in Section 3.1. The requirements on spectroscopic classification and uniformity in targeting were presented in Section 3.2.

Significant testing of the LRG and quasar samples has allowed us to determine whether we meet all of the goals. A summary of the target population was outlined in Section 4.2 while the expected rate of fiber efficiencies was presented in Section 4.3. Combining the number density of the parent target population with the tiling statistics, we can predict the number of spectroscopically confirmed targets that can be used as tracers. Pilot studies described in Section 5 produced a sample of more than 10,000 LRG and more than 10,000 quasar spectra from which we can estimate the performance of spectroscopic classification. Assuming a joint LRG and quasar survey covering $7500 \mathrm{deg}^{2}$, we summarize the expected performance of these two target classes with respect to requirements in Table 7. As can be seen, we satisfy the pipeline requirements on both the LRG and quasar samples and we exceed the required number of clustering quasars by $15 \%$. We expect to miss the requirement for the number of new spectroscopically confirmed LRGs by $12 \%$, leading to an expected degradation in BAO signal of roughly $6 \%$ relative to the requirement. The overlap with the high redshift tail of the BOSS CMASS sample increases the total number of $0.6<z<1.0$ passive galaxies by $66 \%$, thus allowing high precision measurements of BAO over this redshift range.

The final ELG selection remains undecided, so it is not yet possible to present the expected performance for this target class. We do have estimates for the number density using several techniques presented in Section 4.2. We present the statistics we can estimate for two of those samples in Table 7. We report the statistics for the Fisher UgrizWl covering $1500 \mathrm{deg}^{2}$ and the high density DECam selection covering $1100 \mathrm{deg}^{2}$. Because the emission lines are narrow, we expect to easily meet the requirement of $<300 \mathrm{~km} \mathrm{~s}^{-1}$ redshift precision, although we do not have a specific estimate at this time. More challenging will be robust identification of sources; catastrophic failures due to line confusion and sky subtraction residuals pose a risk to this class of target. We postpone discussion of the catastrophic failure rate and the expected uniformity until a future publication.

\subsection{Future Improvements in Automated Data Reduction}

The eBOSS spectroscopic pipeline will be based on the BOSS spectroscopic pipeline that was adapted from the original SDSS "idlspec2d" pipeline. It consists of two main parts: the "spectro2d" portion that extracts two-dimensional raw data into one-dimensional flux calibrated spectra; and the "spectro1d" portion that measures scalar quantities such as classifications and redshifts from those one-dimensional spectra (Bolton et al. 2012). For the most part, eBOSS requires the same spectro2d performance as achieved in BOSS, but extended to fainter targets. As discussed in Section 5.1, a number of problems in extraction and/or flux calibration have arisen that degrade the redshift classification. 
New development on the data reduction pipeline has begun to address these problems. This renewed effort includes removing a $\mathrm{S} / \mathrm{N}$ dependent bias in the extractions, coadditions, and sky subtraction; improving the sky model in the presence of variations in brightness and fiber resolution over the focal plane; improving the error propagation in the coadditions; and correcting occasional failures of flux calibration at the extreme red or blue ends of the spectrum. New flux calibration that accounts for ADR across the focal plane (Margala et al. 2015) will also be implemented in the data reduction pipeline. These changes will provide more accurate spectra with better error models. Without making any changes to the spectral templates, we hope to resolve a significant fraction of uncertain spectral classifications for the new faint targets in eBOSS by making these changes to the spectro $2 \mathrm{~d}$ pipeline.

The analysis of quasar Ly $\alpha$ forest beyond the baseline BAO measurements may require further developments to the spectro2d pipeline, though the exact requirements are not specified at this point in time. Characterization of the spectra and development of new algorithms falls under the purview of the Ly $\alpha$ working group and not the team that develops the data reduction pipeline.

Any new developments in the spectro2d component of the data reduction pipeline will be backward compatible with BOSS data, enabling consistent reprocessing for joint analyses of BOSS and eBOSS data. We do not require SDSS-I and SDSS-II data to be re-processed because the smaller wavelength range renders the early $z>2.15$ quasar spectra obsolete with respect to the BOSS and eBOSS spectra. Because of a lack of refined selection techniques and reduced system throughput, no high redshift ELG or LRG spectra are found in the earlier programs.

The spectroscopic classifications and redshifts from spectrold provide the final map for the clustering measurements and must meet the requirements laid out in Section 3.2. As described throughout the text and in Table 7, the BOSS spectral templates are nearly sufficient to meet these goals. BOSS quasar identifications were visually checked by two individuals to unambiguously classify the BOSS quasars to a level of detail not currently possible by automated software. There is a small sample of QSO_CORE objects that have spectra from previous SDSS surveys that were never visually inspected. To guarantee consistency with prior observations, we will visually inspect each of those. The vast number of quasar spectra in eBOSS require robust spectral classification in an automated fashion. However, even with the new redshifting algorithm presented in Section 5.2, 7\% of eBOSS quasar targets will need visual inspection. Given that these will be the most difficult spectra to characterize, the time investment will not be terribly different from that in BOSS. Improvements to the spectral templates are therefore required to improve the quasar classification in an automated fashion. Improvements to the LRG and ELG templates should also mitigate catastrophic failures and provide better discrimination between the true redshift and other local minima in the distribution of fits. In addition to redshifts for clustering measurements, TDSS and SPIDERS will produce a more heterogeneous spectroscopic sample than is expected for the eBOSS LRG, ELG, and quasar clustering samples. Thus new templates will likely be needed to meet the requirements for automated redshift determination for the LRG, ELG, and quasar samples, and to encompass the heterogeneity of the TDSS and SPIDERS data.
The BOSS spectrold pipeline was based upon PCA templates for quasars and galaxies and physically motivated templates for stars. The BOSS quasar templates do not cover the full variation of actual quasars for BOSS, much less the TDSS and SPIDERS samples. Unphysical combinations of the BOSS PCA templates can also lead to erroneous redshift estimates as shown in Section 5.1. We are exploring a purely archetype-based alternative where, by construction, every template is physically meaningful, thus eliminating the false redshift estimates from unphysical combinations of PCA-based templates. We also expect to introduce more linear combinations of templates to allow classification of such as binary stars or AGN with significant galaxy light.

Any new developments in the spectrold component of the data reduction pipeline must be backward compatible with SDSS-I, SDSS-II, and BOSS. This requirement is necessary to ensure that redshifts from all samples including objects observed in SDSS and BOSS can be determined in a consistent fashion for clustering measurements.

The initial updates of spectral templates will be modeled on the SEQUELS data to more accurately represent the true target spectral variations. Redshifts for LRGs, ELGs, and quasars will be determined by visual inspection and used as input in the generation of templates. Subsequent updates of these templates will be possible as more spectra are taken (especially of rarer objects) and any problems in the templates are uncovered. We expect to present new templates in a future publication similar in philosophy to Bolton et al. (2012).

\section{COSMOLOGICAL PROJECTIONS}

Following the methodology developed in Font-Ribera et al. (2014b), we forecast cosmological constraints for the eBOSS program. We begin by using the expected density, redshift distribution, volume, and bias for each tracer to independently predict the constraints on the matter power spectrum at the relevant redshift. From this, we predict the sensitivity of eBOSS to measure the cosmic distance scale through BAO and to measure the rate of structure growth through RSD. We conclude by presenting the power of the combined sample to constrain the properties of dark energy, gravity, neutrino masses, and inflation.

\subsection{Number Density of Spectroscopic Sample}

The density and redshift distributions used in projections are taken from the parent target samples described in Tables 1 and 2. Estimates for the number densities of the final spectroscopic sample are corrected for incompleteness introduced in the tiling process as explained in Section 4.3. The assumed volume density for each spectroscopic sample for the appropriate redshift ranges and the expected survey area is shown in Table 8. In addition to reporting the observed area, we report the total area that meets the requirement for $15 \%$ uniformity in target density. As discussed in Section 3.2, areas with larger fluctuations could be discarded in the cosmology analysis. Because we currently have no way of tracking the regions with excess fluctuations, we do not include the loss of area due to calibration uncertainty. Instead, zeropoint errors will need to be modeled in the analysis. Likewise, the members of the eBOSS collaboration are developing a new bright star mask using the WISE photometry. While the final mask has not been established, it is likely that $3 \%-4 \%$ of the sky will be removed 
Table 8

Expected Volume Density of Each Target Class, Presented in Units $10^{-4} h^{3} \mathrm{Mpc}^{-3}$

\begin{tabular}{|c|c|c|c|c|c|c|c|}
\hline Redshift & $\begin{array}{l}\text { CMASS } \\
\text { LRG }\end{array}$ & $\begin{array}{l}\text { eBOSS } \\
\text { LRG }\end{array}$ & $\begin{array}{l}\text { Clustering } \\
\text { Quasars }^{\mathrm{a}}\end{array}$ & $\begin{array}{c}\text { Ly } \alpha \\
\text { Quasars }^{\text {b }}\end{array}$ & $\begin{array}{l}\text { Fisher } \\
\text { ELG }\end{array}$ & $\begin{array}{l}\text { Low Density } \\
\text { DECam ELG }\end{array}$ & $\begin{array}{l}\text { High Density } \\
\text { DECam ELG }\end{array}$ \\
\hline $0.6<z<0.7$ & 1.137 & 0.810 & 0.119 & $\cdots$ & 1.412 & 0.183 & 0.205 \\
\hline $0.7<z<0.8$ & 0.170 & 0.678 & 0.130 & $\ldots$ & 2.165 & 1.908 & 2.068 \\
\hline $0.8<z<0.9$ & 0.010 & 0.350 & 0.154 & $\cdots$ & 1.654 & 2.673 & 3.034 \\
\hline $0.9<z<1.0$ & 0.001 & 0.097 & 0.171 & $\cdots$ & 0.624 & 1.135 & 1.605 \\
\hline $1.0<z<1.1$ & $\cdots$ & $\cdots$ & 0.163 & $\cdots$ & 0.218 & 0.373 & 0.568 \\
\hline $1.1<z<1.2$ & $\cdots$ & $\cdots$ & 0.170 & $\cdots$ & 0.081 & 0.159 & 0.241 \\
\hline $1.2<z<1.4$ & $\cdots$ & $\cdots$ & 0.175 & $\cdots$ & $\cdots$ & $\ldots$ & $\cdots$ \\
\hline $1.4<z<1.6$ & $\cdots$ & $\cdots$ & 0.166 & $\cdots$ & $\cdots$ & $\cdots$ & $\cdots$ \\
\hline $1.6<z<1.8$ & $\cdots$ & $\cdots$ & 0.151 & $\cdots$ & $\cdots$ & $\cdots$ & $\cdots$ \\
\hline $1.8<z<2.0$ & $\cdots$ & $\cdots$ & 0.137 & $\cdots$ & $\cdots$ & $\cdots$ & $\cdots$ \\
\hline $2.0<z<2.1$ & $\ldots$ & $\ldots$ & 0.122 & $\ldots$ & $\ldots$ & $\ldots$ & $\ldots$ \\
\hline $2.1<z<2.2$ & $\cdots$ & $\cdots$ & 0.093 & $0.069\left(2.75 \mathrm{deg}^{-2}\right)$ & & & \\
\hline $2.2<z<2.4$ & $\cdots$ & $\ldots$ & $\ldots$ & $0.063\left(5.02 \mathrm{deg}^{-2}\right)$ & $\cdots$ & $\cdots$ & $\cdots$ \\
\hline $2.4<z<2.6$ & $\cdots$ & $\ldots$ & $\cdots$ & $0.042\left(3.35 \mathrm{deg}^{-2}\right)$ & $\cdots$ & $\cdots$ & $\cdots$ \\
\hline $2.6<z<2.8$ & $\cdots$ & $\ldots$ & $\cdots$ & $0.027\left(2.16 \mathrm{deg}^{-2}\right)$ & $\ldots$ & $\cdots$ & $\cdots$ \\
\hline $2.8<z<3.0$ & $\cdots$ & $\cdots$ & $\cdots$ & $0.018\left(1.39 \mathrm{deg}^{-2}\right)$ & $\cdots$ & $\cdots$ & $\cdots$ \\
\hline $3.0<z<3.5$ & $\cdots$ & $\cdots$ & $\cdots$ & $0.009\left(1.69 \mathrm{deg}^{-2}\right)$ & $\cdots$ & $\cdots$ & \\
\hline Observed Surface Area & $7000 \mathrm{deg}^{2}$ & $7000 \mathrm{deg}^{2}$ & $7500 \mathrm{deg}^{2}$ & $7500 \mathrm{deg}^{2}$ & $1500 \mathrm{deg}^{2}$ & $1400 \mathrm{deg}^{2}$ & $1100 \mathrm{deg}^{2}$ \\
\hline Uniform Surface Area & $\ldots$ & $6120 \mathrm{deg}^{2}$ & $6750 \operatorname{deg}^{2}$ & $\ldots$ & $\ldots$ & $\ldots$ & $\ldots$ \\
\hline
\end{tabular}

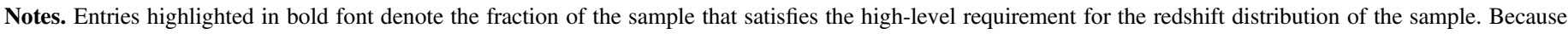
Ly $\alpha$ quasars will be used as random sightlines to projected neutral hydrogen, we report the surface density of those objects parenthetically.

a The clustering quasars are uniformly selected and include BAL and non-BAL quasars previously observed in SDSS-I, -II, and -III.

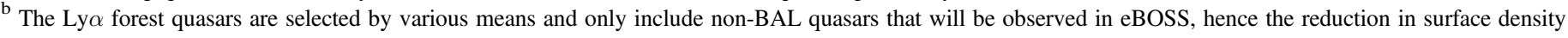
relative to clustering quasars over $2.1<z<2.2$.

due to proximity to bright stars and suspect photometry. Throughout, a flat $\Lambda$ CDM model is assumed with $H_{0}=70$ and $\Omega_{M}=0.31$. These predicted number densities allow us to predict the sensitivity to $D_{A}(z), H(z)$, and $f \sigma_{8}$ from each class of tracer.

We assume $100 \%$ completeness on the clustering quasars observed prior to eBOSS, $95 \%$ completeness on the new targeted clustering quasars, and $100 \%$ completeness on the reobservations of known Ly $\alpha$ quasars. For the PTF-selected quasars, we assume $92 \%$ completeness in fiber assignments and then reduce the density by an additional $10 \%$ to account for incomplete PTF coverage of the eBOSS footprint. We simply average the effect of incomplete coverage over the full area that will be observed.

The surface density of the LRG target sample is taken to be the more conservative estimate presented in Table 1. Because the effective area of the LRG program is reduced by $7 \%$ due to masking by targets tiled in the first round, we take the average density of targets to be the total number of galaxies assigned a fiber over the reduced footprint. We assume a completeness of $87 \%$ of the LRG target sample over a final area of $\sim 7000 \mathrm{deg}^{2}$ for the LRG sample instead of $7500 \mathrm{deg}^{2}$. To account for isolated regions with decollided completeness below $85 \%$ (see Section 4.3 ), we include a $5 \%$ loss of area in addition to the $8 \%$ area that could be lost to targeting non-uniformities when reporting the "Uniform Surface Area" for the LRG sample. Finally, because the high redshift tail overlaps with the new LRG sample, we include the $n(z)$ distribution for CMASS in the redshift range of interest. In the cosmological projections that follow, we combine the two samples for optimal constraints over the redshift range $0.6<z<1.0$.

The ELG program is not yet finalized so we include the volume density for three potential selection schemes. We do not report the results for the gri + Uri selection as it produces tracers at an efficiency of only $52.5 \%$. We assume a completeness of $95 \%$ over $1500 \mathrm{deg}^{2}$ for the Fisher, over $1400 \mathrm{deg}^{2}$ for the low density DECam selections, and over $1100 \mathrm{deg}^{2}$ for the high density DECam selection. As it produces a sample with the highest median redshift, we assume the high density DECam-based ELG selection in the projected BAO and RSD measurements and for the cosmological projections presented in Section 7.3.

\subsection{BAO and RSD Forecasts}

In order to delineate clustering measurements between samples and ensure the predictions are independent, we use only the $0.9<z<2.2$ component of the quasar sample for direct clustering predictions and only the $z>2.1$ component of the quasar sample for $\operatorname{Ly} \alpha$ forest predictions. In reality, the QSO_CORE sample will extend over both redshift regimes and will allow for direct clustering measurements at redshifts beyond those used in these projections.

The Ly $\alpha$ quasar sample in eBOSS will complement the BOSS survey, improving the BAO measurement by providing deeper spectra of known $z>2.1$ quasars and spectra of new $z>2.1$ quasars. As explained in Font-Ribera et al. (2014a), the auto-correlation Ly $\alpha$ forest clustering measurement is shotnoise limited and therefore nearly uncorrelated with the $\operatorname{Ly} \alpha$ forest-quasar cross-correlation measurement. We therefore use the combined precision from these two measurements to forecast the final BAO distance measurement. Rather than applying direct Fisher projections, we instead scale the expected DR12 results according to the scheme presented in Section 5.3. We do this for $H(z)$ and $d_{A}(z)$ but not $R$ because the weighting of the radial modes and transverse modes for 
Table 9

Basic Parameters Expected for Each EBOSS Sample, Together with Predictions for the Effective Volumes and Fractional Constraints on BAO Distance Measurements and Growth of Structure

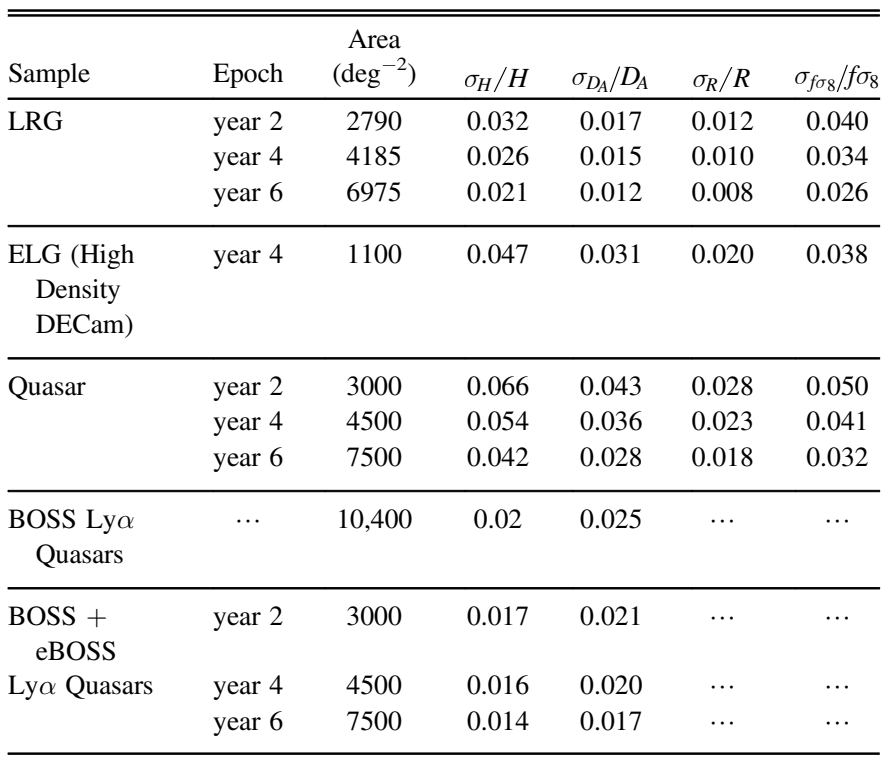

determining the optimal distance indicator is so different between the cross-correlation and auto-correlation analysis. The measurement of RSD from the Ly $\alpha$ sample is considerably more challenging for both theoretical and instrumental considerations, and we do not project its precision in this document.

We present the projections for each sample in two year increments, according to the data release schedule presented in Section 4.5. For BAO forecasts we assume measurements using modes with $k<0.5 h \mathrm{Mpc}^{-1}$; for RSD forecasts we use modes with $k<0.2 \mathrm{~h} \mathrm{Mpc}^{-1}$. This sequence of projections roughly corresponds to the expected schedule for public data releases and the likely publications of clustering measurements. For the LRG, quasar, and Ly $\alpha$ forest samples, we assume that eBOSS will complete $3000 \mathrm{deg}^{2}$ in the first release, an additional $1500 \mathrm{deg}^{2}$ for the second release, and an additional $3000 \mathrm{deg}^{2}$ in the final release. The usable LRG area is somewhat less than the observed area due to loss of targets in regions that are masked by higher priority TDSS, SPIDERS, and quasar targets. We report the area that is expected to be observed in each case. For the ELG sample, we only present the results expected after the second release, in which 300 plates are expected to be observed. The assumed bias for each sample is the same as that presented in the beginning of Section 3.

The time series of predictions on BAO distance precision and RSD growth precision are shown in Table 9. After six years, the LRG sample is expected to produce the highest redshift, sub-percent precision distance measurement obtained by any means. The quasar sample is expected to produce a $1.8 \%$ BAO distance measurement, the first percent-level distance measurement achieved in the interval $1<z<2$. The ELG sample will produce the highest precision BAO measurement to date using this tracer of the matter density field. If one were to assume that the area that fails to meet $15 \%$ uniformity in target selection cannot be used for clustering measurements, the precision of the BAO and RSD measurements will be degraded. For the LRG sample, reducing the area to account for low completeness sectors and excessive
Table 10

Predicted Precision From the Combination of CMB and Large-scale Structure Measurements

\begin{tabular}{lccc}
\hline \hline Parameter & Constraint From & Constraint From & $\begin{array}{c}\text { Constraint From } \\
\text { BOSS, eBOSS, } \\
\text { and CMB }\end{array}$ \\
\hline$\Omega_{M} h^{2}$ & CMB & BOSS and CMB & 0.0017 \\
$w_{0}$ & 0.008 & 0.0028 & 0.15 \\
$w_{a}$ & 0.52 & 0.17 & 0.48 \\
$\gamma$ & 1.4 & 0.67 & 0.10 \\
$\sum m_{\nu}$ & 30.0 & 0.13 & $0.16 \mathrm{eV}$ \\
$n_{s}$ & $0.81 \mathrm{eV}$ & $0.29 \mathrm{eV}$ & 0.0022 \\
\hline
\end{tabular}

Note. All values correspond to the estimated $1-\sigma$ uncertainties.

systematic fluctuations results in a $7 \%$ increase in the projected errors. For the clustering quasar sample, the potential loss of area would result in a $5.5 \%$ increase in the projected errors.

The six-year predictions for the eBOSS distance measurements are shown in Figure 1. It can be seen in the bottom panel that $\mathrm{SNe}$ Ia offer high precision measurements of distance out to redshifts $z<0.6$ or so, while the BAO probes offer constraints comparable to $\mathrm{SNe}$ Ia around $z \sim 0.6$ but offer much stronger constraints for redshifts beyond. As emphasized in Kim et al. (2015), BAO and $\mathrm{SNe}$ provide distinct cosmological constraints even at the same redshift because of the difference between absolute and Hubble flow calibration, making the combination much more powerful than either probe in isolation. The combination of SNe from DES with the final data sets from SNLS and SDSS-II will provide a far larger sample for reducing both statistical and systematic errors. However, because the leading supernova analyses are currently limited by systematic uncertainties (e.g., Sullivan et al. 2011; Betoule et al. 2014), it is difficult to forecast the performance of future surveys.

\subsection{Cosmological Constraints}

In what follows, we report the projections for cosmological constraints from the eBOSS BAO distance, RSD, and power spectrum measurements from the galaxies and quasars using modes with $k<0.1 \mathrm{~h} \mathrm{Mpc}^{-1}$. We report predicted cosmological constraints for a six year program using the combined information of all four tracers. We assume that clustering measurements can be recovered over the areas which display deviations from the uniformity requirement. For these projections, we take the values of the parameters of the fiducial cosmology to be the flat $\Lambda$ CDM model with parameter values as shown in Table 1 of Font-Ribera et al. (2014b). We allow free parameters to describe the growth rate of structure $(\Delta \gamma$ and a normalization constant $G_{9}$ ), the summed neutrino mass $\left(\sum m_{\nu}\right)$, non-Gaussianity in the perturbations of the initial density field $\left(f_{\mathrm{NL}}\right)$, and a time-evolving equation of state for dark energy $\left(w_{0}, w_{a}\right)$. We assume a baseline of Planck CMB measurements, $5 \% H_{0}$ constraint, and the BAO measurements from the complete BOSS galaxy sample. The projected constraints are found in Table 10. A brief interpretation of those predicted constraints in terms of dark energy, modified gravity, neutrinos and inflation is presented below. 


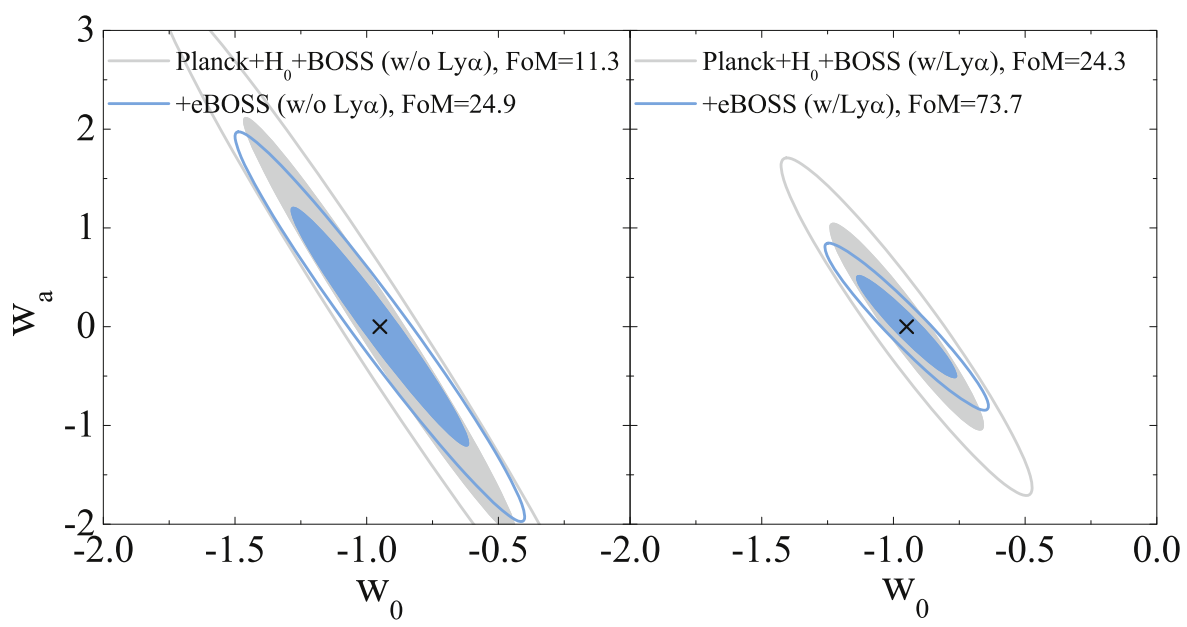

Figure 12. Current constraints on the DETF model for time-varying dark energy compared to projected constraints from eBOSS. We report constraints from the BAO probes, Planck, and $H_{0}$ from $H S T$ observations of SNe Ia and Cepheid variables (Riess et al. 2011). For all measurements, the filled ellipse represents the 68\% confidence interval and the open ellipse represents the $95 \%$ confidence interval.

\subsubsection{Dark Energy}

To demonstrate the power of BAO as an independent probe of the cosmic distance ladder, we report the constraints on the equation of state for dark energy using BOSS and eBOSS BAO measurements as the sole source of information from cosmic times between the CMB and today. We fix neutrino mass and inflation parameters to highlight the relative constraints on dark energy. We predict constraints on a standard parameterization of dark energy in which the equation of state evolves with time as $w(a)=w_{0}+w_{a}(1-a)$. The prediction for the combined constraints on $w_{0}$ and $w_{a}$ is shown in Figure 12 .

\subsubsection{Modified Gravity}

At a given redshift, RSD measurements constrain the product $f \sigma_{8}$, but the extended redshift range of the combined eBOSS and BOSS measurements will significantly reduce the degeneracy between $f$ and $\sigma_{8}$. In predicting the power of eBOSS RSD measurements to constrain models of modified gravity, we assume a parameterization $f(z)=\Omega_{M}^{\gamma}(z)$, where $\gamma$ is the growth index (Linder 2005). The value of $\gamma$ is approximately 0.55 when predicting the growth rate from GR. Measurements with RSD that differ from this value would indicate a model for gravity that deviates from GR at cosmological scales. The amplitude of $f(z)$ is normalized according to a free parameter referenced as $G_{9}$ in the assumed model.

An example of the power of RSD is shown in Figure 2, where the growth rates are compared for a set of cosmological models that predict the same background expansion (i.e., the same distance-redshift relation and comoving BAO position). In the $\Lambda$ CDM model, which assumes that GR correctly describes gravity on all scales, the evolution of $f \sigma_{8}$ is determined entirely by the expansion history $H(z)$. On the other hand, if GR requires modification on cosmological scales, as demonstrated in the other two models plotted, then measurements of $f \sigma_{8}$ over a large redshift interval will reveal that inconsistency, thus indicating that cosmic acceleration arises from modified gravity instead of dark energy. The eBOSS measurements will significantly improve the constraints on $f \sigma_{8}$ between $0.6<z<2.0$, which will help constrain the amplitude of the growth rate at fairly early times where various models nearly converge. More generally, the combination of BAO and RSD measurements will enable eBOSS to simultaneously constrain models of dark energy and modified gravity (e.g., Song \& Percival 2009). A review of the power of eBOSS and other spectroscopic surveys to measure the growth of structure in tandem with imaging surveys is presented in Huterer et al. (2015).

\subsubsection{Neutrinos}

A global fit to solar and atmospheric neutrino flavor oscillations implies a difference in the squares of masses $m_{2}^{2}-m_{1}^{2}=7.54_{-0.22}^{+0.26} \times 10^{-5} \mathrm{eV}^{2}$ and $\mid\left(m_{3}^{2}-m_{1}^{2}\right) / 2+$ $\left(m_{3}^{2}-m_{2}^{2}\right) / 2 \mid=2.42_{-0.11}^{+0.07} \times 10^{-3} \mathrm{eV}^{2}$ (Fogli et al. 2012). Assuming a normal hierarchy in which the lightest neutrino is massless, one can infer the minimum sum of the masses to be greater than $0.05 \mathrm{eV}$. In the case of a so-called inverted hierarchy, where $m_{3}=0$, the minimum sum of the masses must be greater than $0.1 \mathrm{eV}$. Both of these are well below the reach of the present terrestrial experiments. For example, betadecay experiments currently constrain the effective electronneutrino mass to be $\lesssim 2 \mathrm{eV}$ (Otten \& Weinheimer 2008) and even the next generation direct tritium decay experiments such as the Karlsruhe Tritium Neutrino experiment (KATRIN; KATRIN collaboration et al. 2001; Wolf 2010) will only have a sensitivity to constrain $m\left(\nu_{e}\right)<0.2 \mathrm{eV}$ (90\% upper limit). When considering the small mass differences derived from the oscillation experiments, the projected results from KATRIN can at best constrain each individual mass eigenstate to $m<0.2 \mathrm{eV}$, or $\sum m_{\nu}<0.6 \mathrm{eV}$ (90\% confidence).

The large effective volume covered by eBOSS makes it sensitive to neutrino masses. The signature of neutrinos appear as modulations of clustering in the same large-scale structure maps used for BAO and RSD constraints. An example of the predicted suppression of power by massive neutrinos is shown in Figure 13. eBOSS will therefore place tight new constraints on this fundamental quantity without any changes to the BAO survey design. The relative impact of eBOSS, CMB and other cosmology programs to improve neutrino constraints is reviewed in Abazajian et al. (2015b).

The best current cosmological constraints on $\sum m_{\nu}$ from large-scale clustering result from the combination of Planck $\mathrm{CMB}, \mathrm{CMB}$ polarization, lensing and BAO. As reported in 


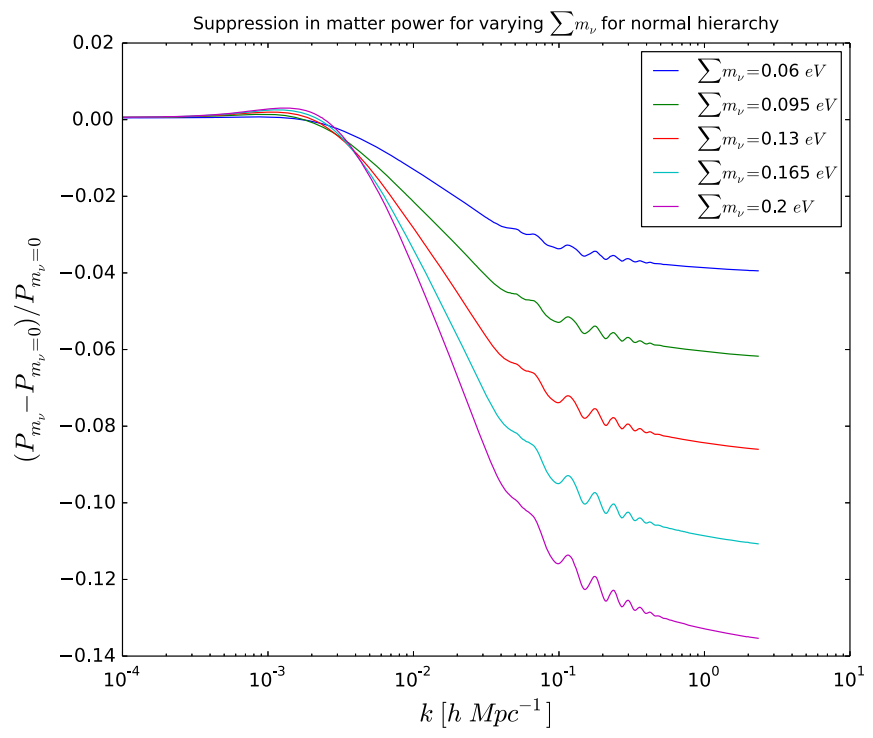

Figure 13. The suppression of power at small angular scales due to freestreaming of massive neutrinos. A normal hierarchy for neutrino masses is assumed.

Planck Collaboration et al. (2015b), these combined probes produce an upper limit $\sum m_{\nu}<0.23 \mathrm{eV}$ (95\% confidence) when assuming zero curvature. With eBOSS, we can provide comparable constraints if one combines information derived from the shape of the broadband power spectrum (for neutrino masses) with the distance measurements derived from BAO. Estimates from the clustering of BOSS galaxies have already shown the potential in spectroscopic surveys to constrain the neutrino mass (e.g., Zhao et al. 2013; Beutler et al. 2014a).

The projections in Table 10 include an estimated precision on the neutrino mass that is highly degenerate with the modified gravity parameters. If one were to assume a flat, $\Lambda$ CDM cosmology described by GR on cosmological scales, we predict a 95\% upper limit $\sum m_{\nu}<0.104 \mathrm{eV}$ when combining the results from the BOSS, eBOSS, and CMB. The reported constraint assumes that that measurements of large-scale modes can be made with the eBOSS galaxies and quasars at all scales larger than $k_{\max }=0.1 \mathrm{~h} \mathrm{Mpc}^{-1}$. If one were to assume measurements of large-scale modes with wavelengths up to $k_{\max }=0.2 \mathrm{~h} \mathrm{Mpc}^{-1}$, the projections for the $95 \%$ upper limit improve to $\sum m_{\nu}<0.072 \mathrm{eV}$. The statistical power of eBOSS can potentially provide an upper limit on neutrino masses comparable to the minimum allowed mass in an inverted hierarchy.

An alternative way to constrain neutrino masses with optical spectroscopy is to use the information from the 1D flux power spectrum of Ly $\alpha$ forest. Measurements of the 1D flux power spectrum using BOSS quasars (Palanque-Delabrouille et al. 2013b) yield the tightest constraints to date using any cosmological tracer. When combining the BOSS measurement with $\mathrm{CMB}$ and $\mathrm{BAO}$ measurements, the sum of the neutrino masses is constrained with a $95 \%$ upper limit $\sum m_{\nu}<0.15 \mathrm{eV}$ (Palanque-Delabrouille et al. 2015b), improved to $\sum m_{\nu}<0.12 \mathrm{eV}$ (Palanque-Delabrouille et al. 2015a). We do not include projections for new constraints from 1D power on the neutrino mass from eBOSS. The deeper spectra obtained by reobserving $1.2 \mathrm{deg}^{-2}$ known quasars and the discovery of $1.2 \mathrm{deg}^{-2}$ new quasars at $z>3$ should allow tighter constraints on the redshift evolution of the 1D flux power spectrum, and thus tighter constraints on the neutrino mass.

\subsubsection{Inflation}

Inflation is the best candidate that we have for a theory that simultaneously established the initial conditions for structure formation while producing a homogeneous, nearly flat universe. Inflation can explain small super-horizon fluctuations which are nearly Gaussian and scale-independent. A review of inflation and the potential of upcoming cosmology programs to improve inflationary models is found in Abazajian et al. (2015a).

The eBOSS survey volume provides sensitivity to the standard inflationary parameters such as the spectral index of primordial matter fluctuations $\left(n_{s}\right)$, defined according to $P(k)=k^{n_{s}}$. Most inflationary models predict a value of $n_{s}$ slightly less than one. One possible departure from standard inflationary scenarios is expected to appear as small deviations from Gaussian fluctuations in the initial density field. This signature of non-Gaussianity in the primordial fluctuations could also be visible in the large-scale structure maps from eBOSS.

A form of potential non-Gaussianity that arises naturally in many inflationary models (Wands 2010) is the "local" form, with a gravitational potential described by

$$
\phi=\phi_{g}+f_{\mathrm{NL}}\left(\phi_{g}^{2}-\left\langle\phi_{g}^{2}\right\rangle\right)
$$

where $\phi_{g}$ is an auxiliary Gaussian field. In the limit of $f_{\mathrm{NL}}=0$, one recovers the Gaussian case. It has been shown (Dalal et al. 2008) that such non-Gaussianity will generate a deviation from the standard prediction in which galaxies are a linear tracer of the underlying dark matter field on large scales. This correction scales inversely as the square of the wavenumber in the matter power spectrum, and it thus becomes important on the larges scales - precisely in the limit where nonlinearities in the dark-matter fluctuations are negligible.

We find that primordial non-Gaussianities of the local form can be constrained to a precision $\sigma_{f_{\mathrm{NL}}}=12$ (68\% confidence) by the combination of eBOSS LRG, ELG, and quasars. This measurement will be independent of the current Planck bispectrum limits of $f_{\mathrm{NL}}=0.8 \pm 5 . \quad(68 \%$ confidence $)$ (Planck 2015 results. XXVII; Planck Collaboration et al. 2015a). While we do not have forecasts for constraints arising from measurements of the galaxy bispectrum, we expect these to significantly tighten the constraints from eBOSS, provided systematic effects can be brought under control (e.g., Ross et al. 2013a). In fact, the first analysis of the BOSS galaxy bispectrum (Gil-Marín et al. 2015a) and cosmological interpretation (Gil-Marín et al. 2015b) were completed in 2014.

\section{CONCLUSION}

As described throughout this paper, the eBOSS survey design has been thoroughly evaluated and should meet a highlevel goal of precise clustering measurements using four distinct tracers over four different redshift intervals. A sample of $52 \mathrm{deg}^{-2}$ targets will be observed and will produce more than $35 \mathrm{deg}^{-2}$ LRGs with confident spectroscopic redshifts between $0.6<z<1.0$. When combined with the $z>0.6$ tail of the BOSS CMASS sample, this LRG sample has the statistical power to constrain the matter power spectrum at 
BAO scales for better than a $1 \%$ distance measurement. Similarly, a uniform sample of quasar candidates selected at a density of $115 \mathrm{deg}^{-2}$ can be efficiently identified. Roughly 30 targets $\mathrm{deg}^{-2}$ have confident spectroscopic classification from SDSS-I, -II, or -III, revealing quasars at a density $13 \mathrm{deg}^{-2}$ between $0.9<z<2.2$. New observations will produce an average of $56 \mathrm{deg}^{-2}$ new quasars at $0.9<z<2.2$. The final sample should be sufficient for a $1.8 \%$ BAO distance measurement. The range of redshifts covered by the LRG and quasar program is only sparsely sampled by supernova surveys, but it covers the crucial epoch of transition from decelerating cosmic expansion to accelerating expansion. As with BOSS, the area covered by the LRG and quasar clustering samples approaches the limits accessible by ground-based telescopes: we will observe one-third of the extra-Galactic sky.

eBOSS will use a deeper sample of Ly $\alpha$ forest observations to improve the BOSS BAO distance measurements by a factor of 1.44 and will introduce a new sample of highly biased ELG targets. Several potential ELG programs have been identified that can provide $\mathrm{BAO}$ measurements at $2 \%$ precision. The final ELG program will be decided when the imaging data sets are better understood and when uniformity of the target selection is fully assessed. Beyond BAO, the clustering in LRG, ELG, quasars, and the $\operatorname{Ly} \alpha$ forest provide broadband power spectra to further explore the cosmological model, particularly in the realm of modified gravity and neutrino mass estimates. The primary analysis effort within the eBOSS collaboration will be to mitigate systematic errors due to the non-uniformities in the target selection and improve modeling of the observed power spectrum at small scales to capitalize on this statistical power.

The cosmological projections presented in Section 7.3 assume a standard cosmological model. Because of the overlap in redshift range between the ELG sample and the LRG and quasar samples, the eBOSS data also enable techniques such as those introduced in McDonald \& Seljak (2009) and Seljak (2009) to combine multiple tracers and reduce the effects of sample variance. Projections for $f_{\mathrm{NL}}$ and RSD from eBOSS following the multi-tracer technique are found in the work by Zhao et al. (2015). Zhao et al. (2015) also present Fisher forecasts for the dark energy equation of state and modified gravity based on a principal component analysis parameterization.

eBOSS will provide unique spectroscopic information that will complement imaging cosmological surveys such as DES. Most imaging probes of dark energy rely on photometric redshift estimates which must be calibrated to extremely high accuracy to avoid degradation in dark energy inference. This calibration is typically done using spectroscopically calibrated redshifts of objects spanning the photometric properties of the sample of interest. However, existing spectroscopic surveys are inherently limited by the difficulty of securing redshifts for faint, high redshift galaxies. Spectroscopy from eBOSS offers an alternative approach to photometric redshift calibration through cross-correlation techniques (Newman 2008; Ménard et al. 2013). The observed degree of correlation between a wellmeasured spectroscopic sample and a photometrically selected sample provides information on the fraction of the imaging sample at that redshift. The possibility of cross-correlation as a tool to calibrate photometric redshifts for DES was the primary reason that the $500 \mathrm{deg}^{2}$ eboss 2 region was tiled in the first year (see Figure 3).
Improvements in the automated data reduction pipeline are underway to ensure that we meet the redshift efficiencies required to meet the assumed cosmological precision. The introduction of new spectral templates that cover a more limited parameter space should significantly improve the ability of the pipeline to differentiate the best fit redshift from contaminating interlopers. When complete, the classifications will produce the largest sample of $z>0.6 \mathrm{LRG}$ to date, a high-redshift ELG sample exceeding that of the WiggleZ Dark Energy Survey (Drinkwater et al. 2010; Blake et al. 2011a), a sample of quasars exceeding that of all previous observations combined, and an enhanced sample of $\operatorname{Ly} \alpha$ forest spectra. These new samples will allow studies of galaxy evolution and quasar astrophysics well beyond the cosmological studies that drove the survey design.

Several studies of galaxy evolution and quasar astrophysics have already begun with the early release of the SEQUELS sample in DR12 and the first eBOSS data taken in Fall 2014. At the redshifts of the LRG and quasar samples, many absorption lines are accessible in the eBOSS spectra. Measurements of quasar absorption features in the vicinity of BOSS spectroscopic galaxies revealed the $\mathrm{Mg}$ II distribution surrounding LRGs at redshifts as low as $z=0.5$ (Zhu et al. 2014). Composite spectra constructed from Ly $\alpha$ forest absorbers shed light on circumgalactic regions and the intergalactic medium (Pieri et al. 2014). These studies will be extended to higher redshift with the eBOSS data. Studies of the correlations in the Ly $\alpha$ forest with other systems are underway following early studies in BOSS (e.g., Font-Ribera et al. 2012), while others have the shown the potential of eBOSS to offer new probes of large-scale structure and BAO (e.g., Pieri 2014). The composite spectrum of ELGs from limited pilot observations already shows rich spectral features such as resonant absorption and non-resonant emission. The first science result from the eBOSS program resulted from studies of this composite ELG spectrum (Zhu et al. 2015). These features are rare in other spectroscopic samples; eBOSS will allow systematic investigation of these features to explore the effect of gas processes on galaxy evolution. The overlap in spectroscopic area covered by eBOSS and wide-field far-infrared imaging from the Herschel Space Telescope (Pilbratt et al. 2010) will enable an exploration of the far-IR luminosity function of the quasar host galaxies. Finally, the quasar sample will greatly exceed all prior work both in total numbers and in the coverage of luminosity-redshift phase space. The sample can be used to enhance BOSS constraints on the luminosity function (e.g., McGreer et al. 2013; Ross et al. 2013b), provide halo occupation statistics that can be used to constrain the duty cycle of quasars as a function of halo mass and quasar luminosity, and to explore the redshift and luminosity evolution of quasars. When complete, the eBOSS spectroscopic sample will results in a diverse range of findings both for cosmology and for galaxy and quasar science.

K.D. acknowledges support from the U.S. Department of Energy under Grant DE-SC000995. J.P.K. and T.D. acknowledge support from the ERC advanced grant LIDA. W.J.P. acknowledges support from the UK STFC through the consolidated grant ST/K0090X/1, and from the European Research Council through grant Darksurvey. This paper includes targets derived from the images of the Wide-Field Infrared Survey Explorer, which is a joint project of the 
University of California, Los Angeles, and the Jet Propulsion Laboratory/California Institute of Technology, funded by the National Aeronautics and Space Administration.

This paper represents an effort by both the SDSS-III and SDSS-IV collaborations. Funding for SDSS-III was provided by the Alfred P. Sloan Foundation, the Participating Institutions, the National Science Foundation, and the U.S. Department of Energy Office of Science. Funding for the Sloan Digital Sky Survey IV has been provided by the Alfred P. Sloan Foundation, the U.S. Department of Energy Office of Science, and the Participating Institutions. SDSS-IV acknowledges support and resources from the Center for HighPerformance Computing at the University of Utah. The SDSS web site is www.sdss.org.

SDSS-IV is managed by the Astrophysical Research Consortium for the Participating Institutions of the SDSS Collaboration including the Brazilian Participation Group, the Carnegie Institution for Science, Carnegie Mellon University, the Chilean Participation Group, the French Participation Group, Harvard-Smithsonian Center for Astrophysics, Instituto de Astrofisica de Canarias, The Johns Hopkins University, Kavli Institute for the Physics and Mathematics of the universe (IPMU)/University of Tokyo, Lawrence Berkeley National Laboratory, Leibniz Institut für Astrophysik Potsdam (AIP), Max-Planck-Institut für Astronomie (MPIA Heidelberg), MaxPlanck-Institut für Astrophysik (MPA Garching), Max-PlanckInstitut für Extraterrestrische Physik (MPE), National Astronomical Observatory of China, New Mexico State University, New York University, University of Notre Dame, Observatário Nacional/MCTI, The Ohio State University, Pennsylvania State University, Shanghai Astronomical Observatory, United Kingdom Participation Group, Universidad Nacional Autónoma de México, University of Arizona, University of Colorado Boulder, University of Portsmouth, University of Utah, University of Virginia, University of Washington, University of Wisconsin, Vanderbilt University, and Yale University.

\section{REFERENCES}

Abazajian, K. N., Adelman-McCarthy, J. K., Agüeros, M. A., et al. 2009, ApJS, 182, 543

Abazajian, K. N., Arnold, K., Austermann, J., et al. 2015a, APh, 63, 55 Abazajian, K. N., Arnold, K., Austermann, J., et al. 2015b, APh, 63, 66 Ahn, C. P., Alexandroff, R., Allende Prieto, C., et al. 2012, ApJS, 203, 21 Alam, S., Albareti, F. D., Allende Prieto, C., et al. 2015a, ApJS, 219, 12 Alam, S., Ho, S., Vargas-Magaña, M., \& Schneider, D. P. 2015b, MNRAS, 453, 1754

Albrecht, A., Bernstein, G., Cahn, R., et al. 2006, arXiv:astro-ph/0609591 Anderson, L., Aubourg, E., Bailey, S., et al. 2012, MNRAS, 427, 3435 Anderson, L., Aubourg, É, Bailey, S., et al. 2014a, MNRAS, 441, 24 Anderson, L., Aubourg, E., Bailey, S., et al. 2014b, MNRAS, 439, 83 Aubourg, É, Bailey, S., Bautista, J. E., et al. 2015, PhRvD, 92, 123516 Bautista, J. E., Bailey, S., Font-Ribera, A., et al. 2015, JCAP, 5, 60 Becker, R. H., White, R. L., \& Helfand, D. J. 1995, ApJ, 450, 559 Bertin, E., \& Arnouts, S. 1996, A\&AS, 117, 393

Betoule, M., Kessler, R., Guy, J., et al. 2014, A\&A, 568, A22 Beutler, F., Blake, C., Colless, M., et al. 2011, MNRAS, 416, 3017 Beutler, F., Blake, C., Colless, M., et al. 2012, MNRAS, 423, 3430 Beutler, F., Saito, S., Brownstein, J. R., et al. 2014a, MNRAS, 444, 3501 Beutler, F., Saito, S., Seo, H.-J., et al. 2014b, MNRAS, 443, 1065 Blake, C., Brough, S., Colless, M., et al. 2012, MNRAS, 425, 405 Blake, C., Davis, T., Poole, G. B., et al. 2011a, MNRAS, 415, 2892 Blake, C., Kazin, E. A., Beutler, F., et al. 2011b, MNRAS, 418, 1707 Blanton, M. R., Lin, H., Lupton, R. H., et al. 2003, AJ, 125, 2276 Blomqvist, M., Kirkby, D., Bautista, J. E., et al. 2015, JCAP, 11, 34 Bolton, A. S., Schlegel, D. J., Aubourg, É, et al. 2012, AJ, 144, 144 Bovy, J., Hennawi, J. F., Hogg, D. W., et al. 2011a, ApJ, 729, 141
Bovy, J., Hogg, D. W., \& Roweis, S. T. 2011b, AnApS, 5, 1657 Bovy, J., Myers, A. D., Hennawi, J. F., et al. 2012, ApJ, 749, 41 Bundy, K., Bershady, M. A., Law, D. R., et al. 2015, ApJ, 798, 7 Busca, N. G., Delubac, T., Rich, J., et al. 2013, A\&A, 552, A96 Chevallier, M., \& Polarski, D. 2001, IJMPD, 10, 213 Chuang, C.-H., Prada, F., Cuesta, A. J., et al. 2013, MNRAS, 433, 3559 Cole, S., Percival, W. J., Peacock, J. A., et al. 2005, MNRAS, 362, 505 Comparat, J., Delubac, T., Jouvel, S., et al. 2015a, arXiv:1509.05045 Comparat, J., Jullo, E., Kneib, J.-P., et al. 2013a, MNRAS, 433, 1146 Comparat, J., Kneib, J.-P., Escoffier, S., et al. 2013b, MNRAS, 428, 1498 Comparat, J., Richard, J., Kneib, J.-P., et al. 2015b, A\&A, 575, A40 Conley, A., Guy, J., Sullivan, M., et al. 2011, ApJS, 192, 1 Croom, S. M., Boyle, B. J., Shanks, T., et al. 2005, MNRAS, 356, 415 Dalal, N., Doré, O., Huterer, D., \& Shirokov, A. 2008, PhRvD, 77, 123514 Dawson, K. S., Schlegel, D. J., Ahn, C. P., et al. 2013, AJ, 145, 10 de la Torre, S., Guzzo, L., Peacock, J. A., et al. 2013, A\&A, 557, A54 Delubac, T., et al. 2016, in preparation

Delubac, T., Bautista, J. E., Busca, N. G., et al. 2015, A\&A, 574, A59 Drinkwater, M. J., Jurek, R. J., Blake, C., et al. 2010, MNRAS, 401, 1429 Eisenstein, D. J., Annis, J., Gunn, J. E., et al. 2001, AJ, 122, 2267 Eisenstein, D. J., Weinberg, D. H., Agol, E., et al. 2011, AJ, 142, 72 Eisenstein, D. J., Zehavi, I., Hogg, D. W., et al. 2005, ApJ, 633, 560 Finkbeiner, D. P., Schlafly, E. F., Schlegel, D. J., et al. 2015, arXiv:1512.01214 Fisher, R. A. 1936, Annals of Eugenics, 7, 179

Fitzpatrick, E. L. 1999, PASP, 111, 63

Flaugher, B. L., Abbott, T. M. C., Angstadt, R., et al. 2012, Proc. SPIE, 8446,11

Fogli, G. L., Lisi, E., Marrone, A., et al. 2012, PhRvD, 86, 013012 Font-Ribera, A., Arnau, E., Miralda-Escudé, J., et al. 2013, JCAP, 5, 18 Font-Ribera, A., Kirkby, D., Busca, N., et al. 2014a, JCAP, 5, 27 Font-Ribera, A., McDonald, P., Mostek, N., et al. 2014b, JCAP, 5, 23 Font-Ribera, A., Miralda-Escudé, J., Arnau, E., et al. 2012, JCAP, 11, 59 Frieman, J. A., Bassett, B., Becker, A., et al. 2008, AJ, 135, 338 Fukugita, M., Ichikawa, T., Gunn, J. E., et al. 1996, AJ, 111, 1748 Gil-Marín, H., Noreña, J., Verde, L., et al. 2015a, MNRAS, 451, 5058 Gil-Marín, H., Verde, L., Noreña, J., et al. 2015b, MNRAS, 452, 1914 Gunn, J. E., Carr, M., Rockosi, C., et al. 1998, AJ, 116, 3040 Gunn, J. E., Siegmund, W. A., Mannery, E. J., et al. 2006, AJ, 131, 2332 Guo, H., Zehavi, I., Zheng, Z., et al. 2013, ApJ, 767, 122 Guo, H., Zheng, Z., Zehavi, I., et al. 2015, MNRAS, 446, 578 Hewett, P. C., \& Wild, V. 2010, MNRAS, 405, 2302

Ho, S., Cuesta, A., Seo, H.-J., et al. 2012, ApJ, 761, 14

Howlett, C., Ross, A. J., Samushia, L., Percival, W. J., \& Manera, M. 2015, MNRAS, 449, 848

Huterer, D., Kirkby, D., Bean, R., et al. 2015, APh, 63, 23

John, T. L. 1988, A\&A, 193, 189

Kaiser, N. 1987, MNRAS, 227, 1

Kaiser, N., Burgett, W., Chambers, K., et al. 2010, Proc. SPIE, 7733, 77330E KATRIN collaboration, 2001, arXiv:hep-ex/0109033

Kim, A. G., Padmanabhan, N., Aldering, G., et al. 2015, APh, 63, 2 Lang, D., Hogg, D. W., \& Schlegel, D. J. 2014, arXiv:1410.7397 Law, N. M., Kulkarni, S. R., Dekany, R. G., et al. 2009, PASP, 121, 1395 Lawrence, A., Warren, S. J., Almaini, O., et al. 2007, MNRAS, 379, 1599 Levi, M., Bebek, C., Beers, T., et al. 2013, arXiv:1308.0847

Linder, E. V. 2003, PhRvL, 90, 091301

Linder, E. V. 2005, PhRvD, 72, 043529

Majewski, S. R., Schiavon, R. P., Frinchaboy, P. M., et al. 2015, arXiv: 1509.05420

Margala, D., Kirkby, D., Dawson, K., et al. 2015, arXiv:1506.04790

Martin, D. C., Fanson, J., Schiminovich, D., et al. 2005, ApJL, 619, L1

McDonald, P., \& Eisenstein, D. J. 2007, PhRvD, 76, 063009

McDonald, P., \& Seljak, U. 2009, JCAP, 10, 7

McGreer, I. D., Jiang, L., Fan, X., et al. 2013, ApJ, 768, 105

McIntosh, D. H., Rix, H.-W., Rieke, M. J., \& Foltz, C. B. 1999, ApJL, 517, L73

McQuinn, M., \& White, M. 2011, MNRAS, 415, 2257

Ménard, B., Scranton, R., Schmidt, S., et al. 2013, arXiv:1303.4722

Merloni, A., Predehl, P., Becker, W., et al. 2012, arXiv:1209.3114

Morganson, E., Green, P. J., Anderson, S. F., et al. 2015, ApJ, 806, 244

Myers, A. D., Brunner, R. J., Nichol, R. C., et al. 2007, ApJ, 658, 85

Myers, A. D., Brunner, R. J., Richards, G. T., et al. 2006, ApJ, 638, 622

Myers, A. D., Palanque-Delabrouille, N., Prakash, A., et al. 2015, ApJS, 221, 27

Newman, J. A. 2008, ApJ, 684, 88

Oka, A., Saito, S., Nishimichi, T., Taruya, A., \& Yamamoto, K. 2014, MNRAS, 439, 2515 
Otten, E. W., \& Weinheimer, C. 2008, RPPh, 71, 086201

Padmanabhan, N., Schlegel, D. J., Finkbeiner, D. P., et al. 2008, ApJ, 674, 1217

Palanque-Delabrouille, N., Magneville, C., Yèche, C., et al. 2013a, A\&A, 551, A29

Palanque-Delabrouille, N., Yèche, C., Baur, J., et al. 2015a, JCAP, 11, 11

Palanque-Delabrouille, N., Yèche, C., Borde, A., et al. 2013b, A\&A, 559, A85

Palanque-Delabrouille, N., Yèche, C., Lesgourgues, J., et al. 2015b, JCAP, 2, 45

Palanque-Delabrouille, N., Yeche, C., Myers, A. D., et al. 2011, A\&A, 530, A122

Pâris, I., et al. 2016, in preparation

Pâris, I., Petitjean, P., Aubourg, É, et al. 2012, A\&A, 548, A66

Pâris, I., Petitjean, P., Aubourg, É, et al. 2014, A\&A, 563, A54

Parkinson, D., Riemer-Sørensen, S., Blake, C., et al. 2012, PhRvD, 86, 103518

Pieri, M. M. 2014, MNRAS, 445, L104

Pieri, M. M., Mortonson, M. J., Frank, S., et al. 2014, MNRAS, 441, 1718

Pilbratt, G. L., Riedinger, J. R., Passvogel, T., et al. 2010, A\&A, 518, L1

Planck Collaboration, Ade, P. A. R., Aghanim, N., et al. 2015a, arXiv: 1502.01592

Planck Collaboration, Ade, P. A. R., Aghanim, N., et al. 2015b, arXiv: 1502.01589

Prakash, A., Licquia, T. C., Newman, J. A., \& Rao, S. M. 2015a, ApJ, 803, 105

Prakash, A., Licquia, T. C., Newman, J. A., et al. 2015b, arXiv:1508.04478

Proga, D. 2003, ApJ, 585, 406

Pullen, A. R., \& Hirata, C. M. 2013, PASP, 125, 705

Raichoor, A., Comparat, J., Delubac, T., et al. 2016, A\&A, 585, A50

Rau, A., Kulkarni, S. R., Law, N. M., et al. 2009, PASP, 121, 1334

Reid, B. A., Samushia, L., White, M., et al. 2012, MNRAS, 426, 2719

Reid, B. A., Seo, H.-J., Leauthaud, A., Tinker, J. L., \& White, M. 2014, MNRAS, 444, 476

Richards, G. T., Kruczek, N. E., Gallagher, S. C., et al. 2011, AJ, 141, 167

Riess, A. G., Macri, L., Casertano, S., et al. 2011, ApJ, 730, 119

Ross, A. J., Ho, S., Cuesta, A. J., et al. 2011, MNRAS, 417, 1350
Ross, A. J., Percival, W. J., Carnero, A., et al. 2013a, MNRAS, 428, 1116

Ross, A. J., Percival, W. J., Sánchez, A. G., et al. 2012a, MNRAS, 424, 564

Ross, A. J., Samushia, L., Burden, A., et al. 2014, MNRAS, 437, 1109

Ross, A. J., Samushia, L., Howlett, C., et al. 2015, MNRAS, 449, 835

Ross, N. P., McGreer, I. D., White, M., et al. 2013b, ApJ, 773, 14

Ross, N. P., Myers, A. D., Sheldon, E. S., et al. 2012b, ApJS, 199, 3

Sako, M., Bassett, B., Becker, A. C., et al. 2014, arXiv:1401.3317

Samushia, L., Reid, B. A., White, M., et al. 2013, MNRAS, 429, 1514

Samushia, L., Reid, B. A., White, M., et al. 2014, MNRAS, 439, 3504

Schlafly, E. F., \& Finkbeiner, D. P. 2011, ApJ, 737, 103

Schlegel, D. J., Finkbeiner, D. P., \& Davis, M. 1998, ApJ, 500, 525

Seljak, U. 2009, PhRvL, 102, 021302

Seo, H.-J., \& Eisenstein, D. J. 2007, ApJ, 665, 14

Shen, Y., Brandt, W. N., Dawson, K. S., et al. 2015, ApJS, 216, 4

Slosar, A., Font-Ribera, A., Pieri, M. M., et al. 2011, JCAP, 9, 1

Slosar, A., Iršič, V., Kirkby, D., et al. 2013, JCAP, 4, 26

Smee, S. A., Gunn, J. E., Uomoto, A., et al. 2013, AJ, 146, 32

Song, Y.-S., \& Percival, W. J. 2009, JCAP, 10, 4

Stern, D., Assef, R. J., Benford, D. J., et al. 2012, ApJ, 753, 30

Sullivan, M., Guy, J., Conley, A., et al. 2011, ApJ, 737, 102

Wands, D. 2010, CQGra, 27, 124002

Weinberg, D. H., Mortonson, M. J., Eisenstein, D. J., et al. 2013, PhR, 530, 87

White, M., Myers, A. D., Ross, N. P., et al. 2012, MNRAS, 424, 933

White, M., Reid, B., Chuang, C.-H., et al. 2015, MNRAS, 447, 234

Wolf, J. \& KATRIN Collaboration. 2010, NIMPA, 623, 442

Wright, E. L., Eisenhardt, P. R. M., Mainzer, A. K., et al. 2010, AJ, 140, 1868

Xu, X., Cuesta, A. J., Padmanabhan, N., Eisenstein, D. J., \& McBride, C. K. 2013, MNRAS, 431, 2834

York, D. G., Adelman, J., Anderson, J. E., et al. 2000, AJ, 120, 1579

Zhao, G.-B., Saito, S., Percival, W. J., et al. 2013, MNRAS, 436, 2038

Zhao, G.-B., Wang, Y., Ross, A. J., et al. 2015, arXiv:1510.08216

Zhu, G., Comparat, J., Kneib, J.-P., et al. 2015, ApJ, 815, 48

Zhu, G., Ménard, B., Bizyaev, D., et al. 2014, MNRAS, 439, 3139 